Please note: An update has been published for this report. To view the update, please click here.

Please note: An erratum has been published for this issue. To view the erratum, please click here. Centers for Disease Control and Prevention

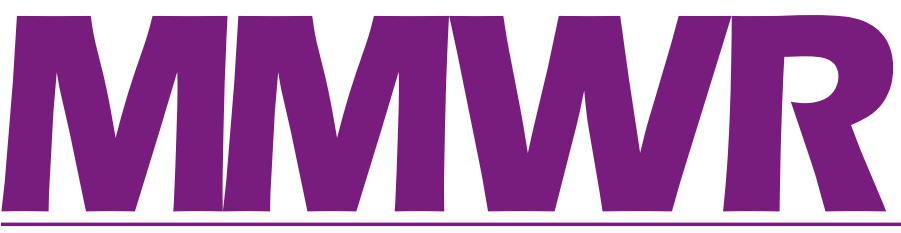

Morbidity and Mortality Weekly Report

Recommendations and Reports / Vol. 65 / No. 1

March 18, 2016

\title{
CDC Guideline for Prescribing Opioids for Chronic Pain — United States, 2016
}
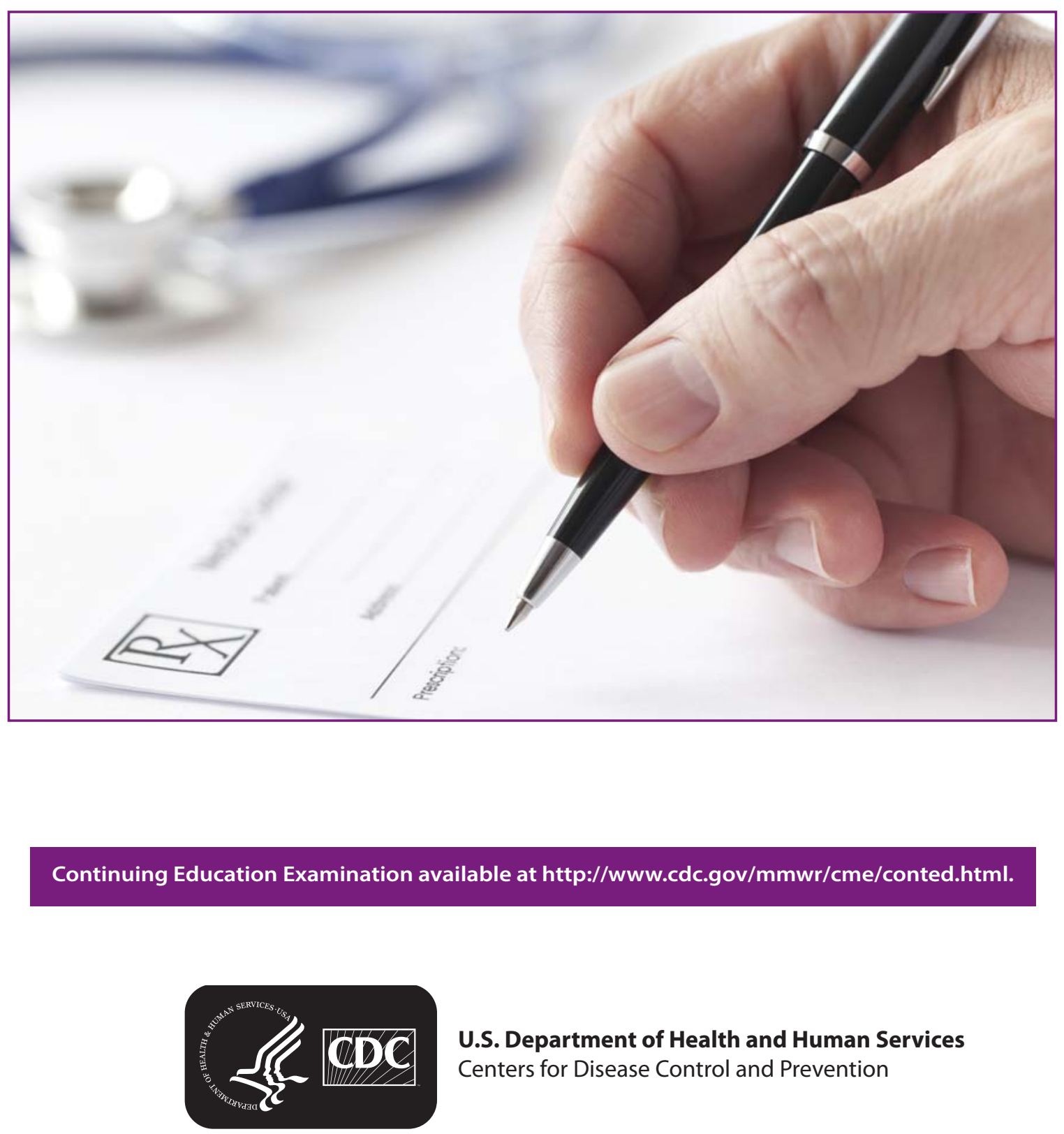


\section{CONTENTS}

Introduction . 1

Guideline Development Methods 4

Summary of the Clinical Evidence Review. . .8

Summary of the Contextual Evidence Review. 11

Recommendations

Conclusions and Future Directions.

References.

\section{Disclosure of Relationship}

The Core Expert Group (CEG) members disclose that they have no financial conflicts of interest. Experts disclose the following activities related to the content of this guideline: Pam Archer discloses authorship of the Oklahoma Emergency Department and Urgent Care Clinic Opioid Prescribing Guidelines and the Opioid Prescribing Guidelines for Oklahoma Health Care Providers in the Office Based Setting; Bonnie Burman discloses authorship of the Ohio Guidelines for Prescribing Opioids for the Treatment of Chronic, Non-Terminal Pain; Jane Ballantyne discloses that she has served as a paid consultant to Cohen Milstein Sellers \& Toll, PLLC, and has special advisory committee responsibilities on the Food and Drug Administration (FDA) Risk Evaluation and Mitigation Strategies committee; Phillip Coffin discloses that in 2012 he provided expert testimony to the California State Assembly regarding a bill to expand naloxone access and reports that he is the principal investigator on a research study of methamphetamine dependence that receives donated injectable naltrexone from Alkermes, Inc.; Gary Franklin discloses authorship of the AMDG Interagency Guideline on Prescribing Opioids for Pain; Erin Krebs discloses that she represented the American College of Physicians at a 2014 Food and Drug Administration meeting on Abuse Deterrent Opioid Formulations; Lewis Nelson discloses his ad-hoc membership on the FDA Drug Safety and Risk Management Advisory Committee; Trupti Patel discloses authorship of the Arizona Opioid Prescribing Guidelines; Robert "Chuck" Rich discloses that he was an author of the 2013 American Academy of Family Physicians position paper on opioids and pain management; Joanna Starrels discloses that she received honoraria from the Betty Ford Institute; Thomas Tape discloses that he was an author of the 2013 American College of Physicians policy position paper on prescription drug abuse. CDC provided $100 \%$ of the funding for the supplemental evidence review tasks and meeting support. No foundation or industry support was accepted.

The Opioid Guideline Workgroup (OGW) members disclose that they have no financial conflicts of interest. Experts disclose the following activities related to the content of this guideline: Anne Burns discloses that she participated in a congressional briefing sponsored by Reps. Carter and DeSaulnier on the pharmacist's role of furnishing Naloxone and that she participates on the National Advisory Board for the Prescription Drug Abuse and Heroin Summit. Chinazo Cunningham discloses that her husband is employed by Quest Diagnostics and Dr. Cunningham was recused from any discussion related to urine drug testing. Traci Green discloses that she was previously employed by Inflexxion, a small business that conducts Small Business Innovation Research on behavioral interventions for behavioral health and chronic pain and created several psychometric tools for conducting risk assessment for prescription opioid abuse potential. Dr. Green also discloses that while at the hospital where she is employed, she provided consultation to Purdue Pharma Ltd to design overdose prevention brochures for persons who use diverted prescription opioids non-medically with an emphasis on persons who inject prescription drugs, and not for patients using opioid therapy for pain. Dr. Green was recused from any discussion related to risk assessment tools and patient education materials. Erin Krebs discloses that she served on the CDC Opioid Prescribing Guideline CEG. Christina Porucznik discloses that she served on the CDC Opioid Prescribing Guideline CEG. Greg Terman discloses that he serves as the President of the American Pain Society. Mark Wallace discloses that he served on a Kempharma advisory panel for an abuse-deterrent hydrocodone formulation to treat acute postoperative pain and Dr. Wallace was recused form any discussion related to abuse-deterrent drugs.

The NCIPC Board of Scientific Counselors (BSC) members disclose that they have no financial conflicts of interest. Two BSC members, Traci Green and Christina Porucznik, served on the Opioid Guideline Workgroup. Traci Green discloses that she was previously employed by Inflexxion, a small business that conducts Small Business Innovation Research on behavioral interventions for behavioral health and chronic pain and created several psychometric tools for conducting risk assessment for prescription opioid abuse potential. Dr. Green also discloses that while at the hospital where she is employed, she provided consultation to Purdue Pharma Ltd to design overdose prevention brochures for persons who use diverted prescription opioids non-medically with an emphasis on persons who inject prescription drugs, and not for patients using opioid therapy for pain. Dr. Green was recused from any discussion related to risk assessment tools and patient education materials. Christina Porucznik discloses that she served on the CDC Opioid Prescribing Guideline CEG.

The MMWR series of publications is published by the Center for Surveillance, Epidemiology, and Laboratory Services, Centers for Disease Control and Prevention (CDC), U.S. Department of Health and Human Services, Atlanta, GA 30329-4027.

Suggested citation: [Author names; first three, then et al., if more than six.] [Title]. MMWR Recomm Rep 2016;65(No. RR-\#):[inclusive page numbers].

\section{Centers for Disease Control and Prevention}

Thomas R. Frieden, MD, MPH, Director

Harold W. Jaffe, MD, MA, Associate Director for Science

Joanne Cono, MD, ScM, Director, Office of Science Quality

Chesley L. Richards, MD, MPH, Deputy Director for Public Health Scientific Services

Michael F. Iademarco, MD, MPH, Director, Center for Surveillance, Epidemiology, and Laboratory Services

\section{MMWR Editorial and Production Staff (Serials)}

\author{
Sonja A. Rasmussen, MD, MS, Editor-in-Chief \\ Charlotte K. Kent, PhD, MPH, Executive Editor \\ Christine G. Casey, MD, Editor \\ Teresa F. Rutledge, Managing Editor \\ David C. Johnson, Lead Technical Writer-Editor \\ Jeffrey D. Sokolow, MA, Project Editor
}

\section{MMWR Editorial Board}

Timothy F. Jones, MD, Chairman

Matthew L. Boulton, MD, MPH

Virginia A. Caine, MD

Katherine Lyon Daniel, $\mathrm{PhD}$

Jonathan E. Fielding, MD, MPH, MBA

David W. Fleming, MD
William E. Halperin, MD, DrPH, MPH
King K. Holmes, MD, PhD
Robin Ikeda, MD, MPH
Rima F. Khabbaz, MD
Phyllis Meadows, $\mathrm{PhD}, \mathrm{MSN}, \mathrm{RN}$
Jewel Mullen, MD, MPH, MPA

Martha F. Boyd, Lead Visual Information Specialist

Maureen A. Leahy, Julia C. Martinroe,

Stephen R. Spriggs, Moua Yang, Tong Yang, Visual Information Specialists Quang M. Doan, MBA,

Phyllis H. King, Terraye M. Starr,

Information Technology Specialists
Jeff Niederdeppe, $\mathrm{PhD}$

Patricia Quinlisk, MD, MPH

Patrick L. Remington, MD, MPH Carlos Roig, MS, MA

William L. Roper, MD, MPH William Schaffner, MD 


\title{
CDC Guideline for Prescribing Opioids for Chronic Pain - United States, 2016
}

\author{
Prepared by \\ Deborah Dowell, $\mathrm{MD}^{1}$ \\ Tamara M. Haegerich, $\mathrm{PhD}^{1}$ \\ Roger Chou, MD ${ }^{1}$ \\ ${ }^{1}$ Division of Unintentional Injury Prevention, National Center for Injury Prevention and Control, CDC, Atlanta, Georgia
}

\begin{abstract}
Summary
This guideline provides recommendations for primary care clinicians who are prescribing opioids for chronic pain outside of active cancer treatment, palliative care, and end-of-life care. The guideline addresses 1) when to initiate or continue opioids for chronic pain; 2) opioid selection, dosage, duration, follow-up, and discontinuation; and 3) assessing risk and addressing harms of opioid use. CDC developed the guideline using the Grading of Recommendations Assessment, Development, and Evaluation (GRADE) framework, and recommendations are made on the basis of a systematic review of the scientific evidence while considering benefits and harms, values and preferences, and resource allocation. CDC obtained input from experts, stakeholders, the public, peer reviewers, and a federally chartered advisory committee. It is important that patients receive appropriate pain treatment with careful consideration of the benefits and risks of treatment options. This guideline is intended to improve communication between clinicians and patients about the risks and benefits of opioid therapy for chronic pain, improve the safety and effectiveness of pain treatment, and reduce the risks associated with long-term opioid therapy, including opioid use disorder, overdose, and death. CDC has provided a checklist for prescribing opioids for chronic pain (http://stacks.cdc.gov/view/cdc/38025) as well as a website (http://www.cdc.gov/drugoverdose/prescribingresources.html) with additional tools to guide clinicians in implementing the recommendations.
\end{abstract}

\section{Introduction}

\section{Background}

Opioids are commonly prescribed for pain. An estimated $20 \%$ of patients presenting to physician offices with noncancer pain symptoms or pain-related diagnoses (including acute and chronic pain) receive an opioid prescription (1). In 2012, health care providers wrote 259 million prescriptions for opioid pain medication, enough for every adult in the United States to have a bottle of pills (2). Opioid prescriptions per capita increased $7.3 \%$ from 2007 to 2012, with opioid prescribing rates increasing more for family practice, general practice, and internal medicine compared with other specialties (3). Rates of opioid prescribing vary greatly across states in ways that cannot be explained by the underlying health status of the population, highlighting the lack of consensus among clinicians on how to use opioid pain medication (2).

Prevention, assessment, and treatment of chronic pain are challenges for health providers and systems. Pain might go unrecognized, and patients, particularly members of racial and ethnic minority groups, women, the elderly, persons with

Corresponding author: Deborah Dowell, Division of Unintentional Injury Prevention, National Center for Injury Prevention and Control, CDC. E-mail: gdo7@cdc.gov. cognitive impairment, and those with cancer and at the end of life, can be at risk for inadequate pain treatment (4). Patients can experience persistent pain that is not well controlled. There are clinical, psychological, and social consequences associated with chronic pain including limitations in complex activities, lost work productivity, reduced quality of life, and stigma, emphasizing the importance of appropriate and compassionate patient care (4). Patients should receive appropriate pain treatment based on a careful consideration of the benefits and risks of treatment options.

Chronic pain has been variably defined but is defined within this guideline as pain that typically lasts $>3$ months or past the time of normal tissue healing (5). Chronic pain can be the result of an underlying medical disease or condition, injury, medical treatment, inflammation, or an unknown cause (4). Estimates of the prevalence of chronic pain vary, but it is clear that the number of persons experiencing chronic pain in the United States is substantial. The 1999-2002 National Health and Nutrition Examination Survey estimated that $14.6 \%$ of adults have current widespread or localized pain lasting at least 3 months ( 6 ). Based on a survey conducted during 2001-2003 (7), the overall prevalence of common, predominantly musculoskeletal pain conditions (e.g., arthritis, rheumatism, chronic back or neck problems, and frequent severe headaches) was estimated at $43 \%$ among adults in the 
United States, although minimum duration of symptoms was not specified. Most recently, analysis of data from the 2012 National Health Interview Study showed that $11.2 \%$ of adults report having daily pain (8). Clinicians should consider the full range of therapeutic options for the treatment of chronic pain. However, it is hard to estimate the number of persons who could potentially benefit from opioid pain medication long term. Evidence supports short-term efficacy of opioids for reducing pain and improving function in noncancer nociceptive and neuropathic pain in randomized clinical trials lasting primarily $\leq 12$ weeks $(9,10)$, and patients receiving opioid therapy for chronic pain report some pain relief when surveyed (11-13). However, few studies have been conducted to rigorously assess the long-term benefits of opioids for chronic pain (pain lasting $>3$ months) with outcomes examined at least 1 year later (14). On the basis of data available from health systems, researchers estimate that 9.6-11.5 million adults, or approximately $3 \%-4 \%$ of the adult U.S. population, were prescribed long-term opioid therapy in 2005 (15).

Opioid pain medication use presents serious risks, including overdose and opioid use disorder. From 1999 to 2014, more than 165,000 persons died from overdose related to opioid pain medication in the United States (16). In the past decade, while the death rates for the top leading causes of death such as heart disease and cancer have decreased substantially, the death rate associated with opioid pain medication has increased markedly (17). Sales of opioid pain medication have increased in parallel with opioid-related overdose deaths (18). The Drug Abuse Warning Network estimated that $>420,000$ emergency department visits were related to the misuse or abuse of narcotic pain relievers in 2011, the most recent year for which data are available (19). Although clinical criteria have varied over time, opioid use disorder is a problematic pattern of opioid use leading to clinically significant impairment or distress. This disorder is manifested by specific criteria such as unsuccessful efforts to cut down or control use and use resulting in social problems and a failure to fulfill major role obligations at work, school, or home (20). This diagnosis has also been referred to as "abuse or dependence" and "addiction" in the literature, and is different from tolerance (diminished response to a drug with repeated use) and physical dependence (adaptation to a drug that produces symptoms of withdrawal when the drug is stopped), both of which can exist without a diagnosed disorder. In 2013, on the basis of DSM-IV diagnosis criteria, an estimated 1.9 million persons abused or were dependent on prescription opioid pain medication (21). Having a history of a prescription for an opioid pain medication increases the risk for overdose and opioid use disorder (22-24), highlighting the value of guidance on safer prescribing practices for clinicians. For example, a recent study of patients aged 15-64 years receiving opioids for chronic noncancer pain and followed for up to 13 years revealed that one in 550 patients died from opioid-related overdose at a median of 2.6 years from their first opioid prescription, and one in 32 patients who escalated to opioid dosages $>200$ morphine milligram equivalents (MME) died from opioid-related overdose (25).

This guideline provides recommendations for the prescribing of opioid pain medication by primary care clinicians for chronic pain (i.e., pain conditions that typically last $>3$ months or past the time of normal tissue healing) in outpatient settings outside of active cancer treatment, palliative care, and endof-life care. Although the guideline does not focus broadly on pain management, appropriate use of long-term opioid therapy must be considered within the context of all pain management strategies (including nonopioid pain medications and nonpharmacologic treatments). CDC's recommendations are made on the basis of a systematic review of the best available evidence, along with input from experts, and further review and deliberation by a federally chartered advisory committee. The guideline is intended to ensure that clinicians and patients consider safer and more effective treatment, improve patient outcomes such as reduced pain and improved function, and reduce the number of persons who develop opioid use disorder, overdose, or experience other adverse events related to these drugs. Clinical decision making should be based on a relationship between the clinician and patient, and an understanding of the patient's clinical situation, functioning, and life context. The recommendations in the guideline are voluntary, rather than prescriptive standards. They are based on emerging evidence, including observational studies or randomized clinical trials with notable limitations. Clinicians should consider the circumstances and unique needs of each patient when providing care.

\section{Rationale}

Primary care clinicians report having concerns about opioid pain medication misuse, find managing patients with chronic pain stressful, express concern about patient addiction, and report insufficient training in prescribing opioids (26). Across specialties, physicians believe that opioid pain medication can be effective in controlling pain, that addiction is a common consequence of prolonged use, and that long-term opioid therapy often is overprescribed for patients with chronic noncancer pain (27). These attitudes and beliefs, combined with increasing trends in opioid-related overdose, underscore the need for better clinician guidance on opioid prescribing. Clinical practice guidelines focused on prescribing can improve clinician knowledge, change prescribing practices (28), and ultimately benefit patient health. 
Professional organizations, states, and federal agencies (e.g., the American Pain Society/American Academy of Pain Medicine, 2009; the Washington Agency Medical Directors Group, 2015; and the U.S. Department of Veterans Affairs/ Department of Defense, 2010) have developed guidelines for opioid prescribing (29-31). Existing guidelines share some common elements, including dosing thresholds, cautious titration, and risk mitigation strategies such as using risk assessment tools, treatment agreements, and urine drug testing. However, there is considerable variability in the specific recommendations (e.g., range of dosing thresholds of $90 \mathrm{MME} /$ day to $200 \mathrm{MME} /$ day), audience (e.g., primary care clinicians versus specialists), use of evidence (e.g., systematic review, grading of evidence and recommendations, and role of expert opinion), and rigor of methods for addressing conflict of interest (32). Most guidelines, especially those that are not based on evidence from scientific studies published in 2010 or later, also do not reflect the most recent scientific evidence about risks related to opioid dosage.

This CDC guideline offers clarity on recommendations based on the most recent scientific evidence, informed by expert opinion and stakeholder and public input. Scientific research has identified high-risk prescribing practices that have contributed to the overdose epidemic (e.g., highdose prescribing, overlapping opioid and benzodiazepine prescriptions, and extended-release/long-acting [ER/LA] opioids for acute pain) $(24,33,34)$. Using guidelines to address problematic prescribing has the potential to optimize care and improve patient safety based on evidence-based practice (28), as well as reverse the cycle of opioid pain medication misuse that contributes to the opioid overdose epidemic.

\section{Scope and Audience}

This guideline is intended for primary care clinicians (e.g., family physicians and internists) who are treating patients with chronic pain (i.e., pain lasting $>3$ months or past the time of normal tissue healing) in outpatient settings. Prescriptions by primary care clinicians account for nearly half of all dispensed opioid prescriptions, and the growth in prescribing rates among these clinicians has been above average (3). Primary care clinicians include physicians as well as nurse practitioners and physician assistants. Although the focus is on primary care clinicians, because clinicians work within team-based care, the recommendations refer to and promote integrated pain management and collaborative working relationships with other providers (e.g., behavioral health providers, pharmacists, and pain management specialists). Although the transition from use of opioid therapy for acute pain to use for chronic pain is hard to predict and identify, the guideline is intended to inform clinicians who are considering prescribing opioid pain medication for painful conditions that can or have become chronic.

This guideline is intended to apply to patients aged $\geq 18$ years with chronic pain outside of palliative and end-of-life care. For this guideline, palliative care is defined in a manner consistent with that of the Institute of Medicine as care that provides relief from pain and other symptoms, supports quality of life, and is focused on patients with serious advanced illness. Palliative care can begin early in the course of treatment for any serious illness that requires excellent management of pain or other distressing symptoms (35). End-of-life care is defined as care for persons with a terminal illness or at high risk for dying in the near future in hospice care, hospitals, long-term care settings, or at home. Patients within the scope of this guideline include cancer survivors with chronic pain who have completed cancer treatment, are in clinical remission, and are under cancer surveillance only. The guideline is not intended for patients undergoing active cancer treatment, palliative care, or endof-life care because of the unique therapeutic goals, ethical considerations, opportunities for medical supervision, and balance of risks and benefits with opioid therapy in such care.

The recommendations address the use of opioid pain medication in certain special populations (e.g., older adults and pregnant women) and in populations with conditions posing special risks (e.g., a history of substance use disorder). The recommendations do not address the use of opioid pain medication in children or adolescents aged $<18$ years. The available evidence concerning the benefits and harms of long-term opioid therapy in children and adolescents is limited, and few opioid medications provide information on the label regarding safety and effectiveness in pediatric patients. However, observational research shows significant increases in opioid prescriptions for pediatric populations from 2001 to 2010 (36), and a large proportion of adolescents are commonly prescribed opioid pain medications for conditions such as headache and sports injuries (e.g., in one study, $50 \%$ of adolescents presenting with headache received a prescription for an opioid pain medication [37,38]). Adolescents who misuse opioid pain medication often misuse medications from their own previous prescriptions (39), with an estimated $20 \%$ of adolescents with currently prescribed opioid medications reporting using them intentionally to get high or increase the effects of alcohol or other drugs (40). Use of prescribed opioid pain medication before high school graduation is associated with a $33 \%$ increase in the risk of later opioid misuse (41). Misuse of opioid pain medications in adolescence strongly predicts later onset of heroin use (42). Thus, risk of opioid medication use in pediatric populations is of great concern. Additional clinical trial and observational research is needed, 
and encouraged, to inform development of future guidelines for this critical population.

The recommendations are not intended to provide guidance on use of opioids as part of medication-assisted treatment for opioid use disorder. Some of the recommendations might be relevant for acute care settings or other specialists, such as emergency physicians or dentists, but use in these settings or by other specialists is not the focus of this guideline. Readers are referred to other sources for prescribing recommendations within acute care settings and in dental practice, such as the American College of Emergency Physicians' guideline for prescribing of opioids in the emergency department (43); the American Society of Anesthesiologists' guideline for acute pain management in the perioperative setting (44); the Washington Agency Medical Directors' Group Interagency Guideline on Prescribing Opioids for Pain, Part II: Prescribing Opioids in the Acute and Subacute Phase (30); and the Pennsylvania Guidelines on the Use of Opioids in Dental Practice (45). In addition, given the challenges of managing the painful complications of sickle cell disease, readers are referred to the NIH National Heart, Lung, and Blood Institute's Evidence Based Management of Sickle Cell Disease Expert Panel Report for management of sickle cell disease (46).

\section{Guideline Development Methods}

\section{Guideline Development Using the Grading of Recommendations Assessment, Development, and Evaluation Method}

CDC developed this guideline using the Grading of Recommendations Assessment, Development, and Evaluation (GRADE) method (http://www.gradeworkinggroup.org). This method specifies the systematic review of scientific evidence and offers a transparent approach to grading quality of evidence and strength of recommendations. The method has been adapted by the CDC Advisory Committee on Immunization Practices (ACIP) (47). CDC has applied the ACIP translation of the GRADE framework in this guideline. Within the ACIP GRADE framework, the body of evidence is categorized in a hierarchy. This hierarchy reflects degree of confidence in the effect of a clinical action on health outcomes. The categories include type 1 evidence (randomized clinical trials or overwhelming evidence from observational studies), type 2 evidence (randomized clinical trials with important limitations, or exceptionally strong evidence from observational studies), type 3 evidence (observational studies or randomized clinical trials with notable limitations), and type 4 evidence (clinical experience and observations, observational studies with important limitations, or randomized clinical trials with several major limitations). Type of evidence is categorized by study design as well as limitations in study design or implementation, imprecision of estimates, variability in findings, indirectness of evidence, publication bias, magnitude of treatment effects, dose-response gradient, and a constellation of plausible biases that could change observations of effects. Type 1 evidence indicates that one can be very confident that the true effect lies close to that of the estimate of the effect; type 2 evidence means that the true effect is likely to be close to the estimate of the effect, but there is a possibility that it is substantially different; type 3 evidence means that confidence in the effect estimate is limited and the true effect might be substantially different from the estimate of the effect; and type 4 evidence indicates that one has very little confidence in the effect estimate, and the true effect is likely to be substantially different from the estimate of the effect $(47,48)$. When no studies are present, evidence is considered to be insufficient. The ACIP GRADE framework places recommendations in two categories, Category A and Category B. Four major factors determine the category of the recommendation: the quality of evidence, the balance between desirable and undesirable effects, values and preferences, and resource allocation (cost). Category A recommendations apply to all persons in a specified group and indicate that most patients should receive the recommended course of action. Category B recommendations indicate that there should be individual decision making; different choices will be appropriate for different patients, so clinicians must help patients arrive at a decision consistent with patient values and preferences, and specific clinical situations (47). According to the GRADE methodology, a particular quality of evidence does not necessarily imply a particular strength of recommendation (48-50). Category A recommendations can be made based on type 3 or type 4 evidence when the advantages of a clinical action greatly outweigh the disadvantages based on a consideration of benefits and harms, values and preferences, and costs. Category $B$ recommendations are made when the advantages and disadvantages of a clinical action are more balanced. GRADE methodology is discussed extensively elsewhere $(47,51)$. The U.S. Preventive Services Task Force (USPSTF) follows different methods for developing and categorizing recommendations (http://www. uspreventiveservicestaskforce.org). USPSTF recommendations focus on preventive services and are categorized as A, B, C, D, and I. Under the Affordable Care Act, all "nongrandfathered" health plans (that is, those health plans not in existence prior to March 23, 2010 or those with significant changes to their coverage) and expanded Medicaid plans are required to cover 
preventive services recommended by USPSTF with a category $\mathrm{A}$ or $\mathrm{B}$ rating with no cost sharing. The coverage requirements went into effect September 23, 2010. Similar requirements are in place for vaccinations recommended by ACIP, but do not exist for other recommendations made by CDC, including recommendations within this guideline.

A previously published systematic review sponsored by the Agency for Healthcare Research and Quality (AHRQ) on the effectiveness and risks of long-term opioid treatment of chronic pain $(14,52)$ initially served to directly inform the recommendation statements. This systematic clinical evidence review addressed the effectiveness of long-term opioid therapy for outcomes related to pain, function, and quality of life; the comparative effectiveness of different methods for initiating and titrating opioids; the harms and adverse events associated with opioids; and the accuracy of risk-prediction instruments and effectiveness of risk mitigation strategies on outcomes related to overdose, addiction, abuse, or misuse. For the current guideline development, CDC conducted additional literature searches to update the evidence review to include more recently available publications and to answer an additional clinical question about the effect of opioid therapy for acute pain on long-term use. More details about the literature search strategies and GRADE methods applied are provided in the Clinical Evidence Review (http://stacks.cdc.gov/view/cdc/38026). CDC developed GRADE evidence tables to illustrate the quality of the evidence for each clinical question.

As identified in the AHRQ-sponsored clinical evidence review, the overall evidence base for the effectiveness and risks of long-term opioid therapy is low in quality per the GRADE criteria. Thus, contextual evidence is needed to provide information about the benefits and harms of nonpharmacologic and nonopioid pharmacologic therapy and the epidemiology of opioid pain medication overdose and inform the recommendations. Further, as elucidated by the GRADE Working Group, supplemental information on clinician and patient values and preferences and resource allocation can inform judgments of benefits and harms and be helpful for translating the evidence into recommendations. CDC conducted a contextual evidence review to supplement the clinical evidence review based on systematic searches of the literature. The review focused on the following four areas: effectiveness of nonpharmacologic and nonopioid pharmacologic treatments; benefits and harms related to opioid therapy (including additional studies not included in the clinical evidence review such as studies that evaluated outcomes at any duration or used observational study designs related to specific opioid pain medications, high-dose opioid therapy, co-prescription of opioids with other controlled substances, duration of opioid use, special populations, risk stratification/mitigation approaches, and effectiveness of treatments for addressing potential harms of opioid therapy); clinician and patient values and preferences; and resource allocation. CDC constructed narrative summaries of this contextual evidence and used the information to support the clinical recommendations. More details on methods for the contextual evidence review are provided in the Contextual Evidence Review (http://stacks.cdc.gov/view/cdc/38027).

On the basis of a review of the clinical and contextual evidence (review methods are described in more detail in subsequent sections of this report), CDC drafted recommendation statements focused on determining when to initiate or continue opioids for chronic pain; opioid selection, dosage, duration, follow-up, and discontinuation; and assessing risk and addressing harms of opioid use. To help assure the draft guideline's integrity and credibility, CDC then began a multistep review process to obtain input from experts, stakeholders, and the public to help refine the recommendations.

\section{Solicitation of Expert Opinion}

CDC sought the input of experts to assist in reviewing the evidence and providing perspective on how CDC used the evidence to develop the draft recommendations. These experts, referred to as the "Core Expert Group" (CEG) included subject matter experts, representatives of primary care professional societies and state agencies, and an expert in guideline development methodology.* CDC identified subject matter experts with high scientific standing; appropriate academic and clinical training and relevant clinical experience; and proven scientific excellence in opioid prescribing, substance use disorder treatment, and pain management. CDC identified representatives from leading primary care professional organizations to represent the audience for this guideline. Finally, CDC identified state agency officials and representatives based on their experience with state guidelines for opioid prescribing that were developed with multiple agency stakeholders and informed by scientific literature and existing evidence-based guidelines.

Prior to their participation, CDC asked potential experts to reveal possible conflicts of interest such as financial relationships with industry, intellectual preconceptions, or previously stated public positions. Experts could not serve if they had conflicts that might have a direct and predictable effect on the recommendations. CDC excluded experts who had a financial or promotional relationship with a company

\footnotetext{
* A list of the members appears at the end of this report. The recommendations and all statements included in this guideline are those of CDC and do not necessarily represent the official position of any persons or organizations providing comments on the draft guideline.
} 
that makes a product that might be affected by the guideline. CDC reviewed potential nonfinancial conflicts carefully (e.g., intellectual property, travel, public statements or positions such as congressional testimony) to determine if the activities would have a direct and predictable effect on the recommendations. CDC determined the risk of these types of activities to be minimal for the identified experts. All experts completed a statement certifying that there was no potential or actual conflict of interest. Activities that did not pose a conflict (e.g., participation in Food and Drug Administration [FDA] activities or other guideline efforts) are disclosed.

CDC provided to each expert written summaries of the scientific evidence (both the clinical and contextual evidence reviews conducted for this guideline) and CDC's draft recommendation statements. Experts provided individual ratings for each draft recommendation statement based on the balance of benefits and harms, evidence strength, certainty of values and preferences, cost, recommendation strength, rationale, importance, clarity, and ease of implementation. CDC hosted an in-person meeting of the experts that was held on June 23-24, 2015, in Atlanta, Georgia, to seek their views on the evidence and draft recommendations and to better understand their premeeting ratings. CDC sought the experts' individual opinions at the meeting. Although there was widespread agreement on some of the recommendations, there was disagreement on others. Experts did not vote on the recommendations or seek to come to a consensus. Decisions about recommendations to be included in the guideline, and their rationale, were made by CDC. After revising the guideline, CDC sent written copies of it to each of the experts for review and asked for any additional comments; CDC reviewed these written comments and considered them when making further revisions to the draft guideline. The experts have not reviewed the final version of the guideline.

\section{Federal Partner Engagement}

Given the scope of this guideline and the interest of agencies across the federal government in appropriate pain management, opioid prescribing, and related outcomes, CDC invited its National Institute of Occupational Safety and Health and CDC's federal partners to observe the expert meeting, provide written comments on the full draft guideline after the meeting, and review the guideline through an agency clearance process; $\mathrm{CDC}$ reviewed comments and incorporated changes. Interagency collaboration will be critical for translating these recommendations into clinical practice. Federal partners included representatives from the Substance Abuse and Mental Health Services Administration, the National Institute on Drug Abuse, FDA, the U.S. Department of Veterans Affairs, the U.S. Department of Defense, the Office of the National Coordinator for Health Information Technology, the Centers for Medicare and Medicaid Services, the Health Resources and Services Administration, AHRQ, and the Office of National Drug Control Policy.

\section{Stakeholder Comment}

Given the importance of the guideline for a wide variety of stakeholders, CDC also invited review from a Stakeholder Review Group (SRG) to provide comment so that CDC could consider modifications that would improve the recommendations' specificity, applicability, and ease of implementation. The SRG included representatives from professional organizations that represent specialties that commonly prescribe opioids (e.g., pain medicine, physical medicine and rehabilitation), delivery systems within which opioid prescribing occurs (e.g., hospitals), and representation from community organizations with interests in pain management and opioid prescribing.* Representatives from each of the SRG organizations were provided a copy of the guideline for comment. Each of these representatives provided written comments. Once input was received from the full SRG, CDC reviewed all comments and carefully considered them when revising the draft guideline.

\section{Constituent Engagement}

To obtain initial perspectives from constituents on the recommendation statements, including clinicians and prospective patients, CDC convened a constituent engagement webinar and circulated information about the webinar in advance through announcements to partners. CDC hosted the webinar on September 16 and 17, 2015, provided information about the methodology for developing the guideline, and presented the key recommendations. A fact sheet was posted on the CDC Injury Center website (http://www.cdc.gov/ injury) summarizing the guideline development process and clinical practice areas addressed in the guideline; instructions were included on how to submit comments via email. CDC received comments during and for 2 days following the first webinar. Over 1,200 constituent comments were received. Comments were reviewed and carefully considered when revising the draft guideline.

\section{Peer Review}

Per the final information quality bulletin for peer review (https://www.whitehouse.gov/sites/default/files/omb/ memoranda/fy2005/m05-03.pdf), peer review requirements applied to this guideline because it provides influential 
scientific information that could have a clear and substantial impact on public- and private-sector decisions. Three experts independently reviewed the guideline to determine the reasonableness and strength of recommendations; the clarity with which scientific uncertainties were clearly identified; and the rationale, importance, clarity, and ease of implementation of the recommendations. ${ }^{*} \mathrm{CDC}$ selected peer reviewers based on expertise, diversity of scientific viewpoints, and independence from the guideline development process. CDC assessed and managed potential conflicts of interest using a process similar to the one as described for solicitation of expert opinion. No financial interests were identified in the disclosure and review process, and nonfinancial activities were determined to be of minimal risk; thus, no significant conflict of interest concerns were identified. CDC placed the names of peer reviewers on the CDC and the National Center for Injury Prevention and Control Peer Review Agenda websites that are used to provide information about the peer review of influential documents. CDC reviewed peer review comments and revised the draft guideline accordingly.

\section{Public Comment}

To obtain comments from the public on the full guideline, CDC published a notice in the Federal Register (80 FR 77351) announcing the availability of the guideline and the supporting clinical and contextual evidence reviews for public comment. The comment period closed January 13, 2016. CDC received more than 4,350 comments from the general public, including patients with chronic pain, clinicians, families who have lost loved ones to overdose, medical associations, professional organizations, academic institutions, state and local governments, and industry. CDC reviewed each of the comments and carefully considered them when revising the draft guideline.

\section{Federal Advisory Committee Review and Recommendation}

The National Center for Injury Prevention and Control (NCIPC) Board of Scientific Counselors (BSC) is a federal advisory committee that advises and makes recommendations to the Secretary of the Department of Health and Human Services, the Director of CDC, and the Director of NCIPC.* The BSC makes recommendations regarding policies, strategies, objectives, and priorities, and reviews progress toward injury and violence prevention. CDC sought the BSC's advice on the draft guideline. BSC members are special government employees appointed as CDC advisory committee members; as such, all members completed an OGE Form 450 to disclose relevant interests. BSC members also reported on their disclosures during meetings. Disclosures for the BSC are reported in the guideline.

To assist in guideline review, on December 14, 2015, via Federal Register notice, CDC announced the intent to form an Opioid Guideline Workgroup (OGW) to provide observations on the draft guideline to the BSC. CDC provided the BSC with the draft guideline as well as summaries of comments provided to CDC by stakeholders, constituents, and peer reviewers, and edits made to the draft guideline in response. During an open meeting held on January 7, 2016, the BSC recommended the formation of the OGW. The OGW included a balance of perspectives from audiences directly affected by the guideline, audiences that would be directly involved with implementing the recommendations, and audiences qualified to provide representation. The OGW comprised clinicians, subject matter experts, and a patient representative, with the following perspectives represented: primary care, pain medicine, public health, behavioral health, substance abuse treatment, pharmacy, patients, and research.* Additional sought-after attributes were appropriate academic and clinical training and relevant clinical experience; high scientific standing; and knowledge of the patient, clinician, and caregiver perspectives. In accordance with CDC policy, two BSC committee members also served as OGW members, with one serving as the OGW Chair. The professional credentials and interests of OGW members were carefully reviewed to identify possible conflicts of interest such as financial relationships with industry, intellectual preconceptions, or previously stated public positions. Only OGW members whose interests were determined to be minimal were selected. When an activity was perceived as having the potential to affect a specific aspect of the recommendations, the activity was disclosed, and the OGW member was recused from discussions related to that specific aspect of the recommendations (e.g., urine drug testing and abuse-deterrent formulations). Disclosures for the OGW are reported. CDC and the OGW identified ad-hoc consultants to supplement the workgroup expertise, when needed, in the areas of pediatrics, occupational medicine, obstetrics and gynecology, medical ethics, addiction psychiatry, physical medicine and rehabilitation, guideline development methodology, and the perspective of a family member who lost a loved one to opioid use disorder or overdose.

The BSC charged the OGW with reviewing the quality of the clinical and contextual evidence reviews and reviewing each of the recommendation statements and accompanying rationales. For each recommendation statement, the OGW considered the quality of the evidence, the balance of benefits and risks, the values and preferences of clinicians and patients, the cost feasibility, and the category designation 
of the recommendation (A or B). The OGW also reviewed supplementary documents, including input provided by the CEG, SRG, peer reviewers, and the public. OGW members discussed the guideline accordingly during virtual meetings and drafted a summary report of members' observations, including points of agreement and disagreement, and delivered the report to the BSC.

NCIPC announced an open meeting of the NCIPC BSC in the Federal Register on January 11, 2015. The BSC met on January 28, 2016, to discuss the OGW report and deliberate on the draft guideline itself. Members of the public provided comments at this meeting. After discussing the OGW report, deliberating on specific issues about the draft guideline identified at the meeting, and hearing public comment, the BSC voted unanimously: to support the observations made by the OGW; that CDC adopt the guideline recommendations that, according to the workgroup's report, had unanimous or majority support; and that CDC further consider the guideline recommendations for which the group had mixed opinions. CDC carefully considered the OGW observations, public comments, and BSC recommendations, and revised the guideline in response.

\section{Summary of the Clinical Evidence Review \\ Primary Clinical Questions}

CDC conducted a clinical systematic review of the scientific evidence to identify the effectiveness, benefits, and harms of long-term opioid therapy for chronic pain, consistent with the GRADE approach $(47,48)$. Long-term opioid therapy is defined as use of opioids on most days for $>3$ months. A previously published AHRQ-funded systematic review on the effectiveness and risks of long-term opioid therapy for chronic pain comprehensively addressed four clinical questions $(14,52)$. CDC, with the assistance of a methodology expert, searched the literature to identify newly published studies on these four original questions. Because long-term opioid use might be affected by use of opioids for acute pain, CDC subsequently developed a fifth clinical question (last in the series below), and in collaboration with a methodologist conducted a systematic review of the scientific evidence to address it. In brief, five clinical questions were addressed:

- The effectiveness of long-term opioid therapy versus placebo, no opioid therapy, or nonopioid therapy for long term ( $\geq 1$ year) outcomes related to pain, function, and quality of life, and how effectiveness varies according to the type/cause of pain, patient demographics, and patient comorbidities (Key Question [KQ] 1).

- The risks of opioids versus placebo or no opioids on abuse, addiction, overdose, and other harms, and how harms vary according to the type/cause of pain, patient demographics, patient comorbidities, and dose (KQ2).

- The comparative effectiveness of opioid dosing strategies (different methods for initiating and titrating opioids; immediate-release versus ER/LA opioids; different ER/LA opioids; immediate-release plus ER/LA opioids versus ER/LA opioids alone; scheduled, continuous versus as-needed dosing; dose escalation versus dose maintenance; opioid rotation versus maintenance; different strategies for treating acute exacerbations of chronic pain; decreasing opioid doses or tapering off versus continuation; and different tapering protocols and strategies) (KQ3).

- The accuracy of instruments for predicting risk for opioid overdose, addiction, abuse, or misuse; the effectiveness of risk mitigation strategies (use of risk prediction instruments); effectiveness of risk mitigation strategies including opioid management plans, patient education, urine drug testing, prescription drug monitoring program (PDMP) data, monitoring instruments, monitoring intervals, pill counts, and abuse-deterrent formulations for reducing risk for opioid overdose, addiction, abuse, or misuse; and the comparative effectiveness of treatment strategies for managing patients with addiction (KQ4).

- The effects of prescribing opioid therapy versus not prescribing opioid therapy for acute pain on long-term use (KQ5).

The review was focused on the effectiveness of long-term opioid therapy on long-term ( $>1$ year) outcomes related to pain, function, and quality of life to ensure that findings are relevant to patients with chronic pain and long-term opioid prescribing. The effectiveness of short-term opioid therapy has already been established (10). However, opioids have unique effects such as tolerance and physical dependence that might influence assessments of benefit over time. These effects raise questions about whether findings on short-term effectiveness of opioid therapy can be extrapolated to estimate benefits of long-term therapy for chronic pain. Thus, it is important to consider studies that provide data on long-term benefit. For certain opioid-related harms (overdose, fractures, falls, motor vehicle crashes), observational studies were included with outcomes measured at shorter intervals because such outcomes can occur early during opioid therapy, and such harms are not captured well in short-term clinical trials. A detailed listing of the key questions is provided in the Clinical Evidence Review (http://stacks.cdc.gov/view/cdc/38026). 


\section{Clinical Evidence Systematic Review Methods}

Complete methods and data for the 2014 AHRQ report, upon which this updated systematic review is based, have been published previously $(14,52)$. Study authors developed the protocol using a standardized process (53) with input from experts and the public and registered the protocol in the PROSPERO database (54). For the 2014 AHRQ report, a research librarian searched MEDLINE, the Cochrane Central Register of Controlled Trials, the Cochrane Database of Systematic Reviews, PsycINFO, and CINAHL for Englishlanguage articles published January 2008 through August 2014, using search terms for opioid therapy, specific opioids, chronic pain, and comparative study designs. Also included were relevant studies from an earlier review (10) in which searches were conducted without a date restriction, reference lists were reviewed, and ClinicalTrials.gov was searched. CDC updated the AHRQ literature search using the same search strategies as in the original review including studies published before April, 2015. Seven additional studies met inclusion criteria and were added to the review. CDC used the GRADE approach outlined in the ACIP Handbook for Developing Evidence-Based Recommendations (47) to rate the quality of evidence for the full body of evidence (evidence from the 2014 AHRQ review plus the update) for each clinical question. Evidence was categorized into the following types: type 1 (randomized clinical trials or overwhelming evidence from observational studies), type 2 (randomized clinical trials with important limitations, or exceptionally strong evidence from observational studies), type 3 (observational studies, or randomized clinical trials with notable limitations), or type 4 (clinical experience and observations, observational studies with important limitations, or randomized clinical trials with several major limitations). When no studies were present, evidence was considered to be insufficient. Per GRADE methods, type of evidence was categorized by study design as well as a function of limitations in study design or implementation, imprecision of estimates, variability in findings, indirectness of evidence, publication bias, magnitude of treatment effects, dose-response gradient, and constellation of plausible biases that could change effects. Results were synthesized qualitatively, highlighting new evidence identified during the update process. Meta-analysis was not attempted due to the small numbers of studies, variability in study designs and clinical heterogeneity, and methodological shortcomings of the studies. More detailed information about data sources and searches, study selection, data extraction and quality assessment, data synthesis, and update search yield and new evidence for the current review is provided in the Clinical Evidence Review (http://stacks.cdc.gov/view/cdc/38026).

\section{Summary of Findings for Clinical Questions}

The main findings of this updated review are consistent with the findings of the 2014 AHRQ report (14). In summary, evidence on long-term opioid therapy for chronic pain outside of end-of-life care remains limited, with insufficient evidence to determine long-term benefits versus no opioid therapy, though evidence suggests risk for serious harms that appears to be dose-dependent. These findings supplement findings from a previous review of the effectiveness of opioids for adults with chronic noncancer pain. In this previous review, based on randomized trials predominantly $\leq 12$ weeks in duration, opioids were found to be moderately effective for pain relief, with small benefits for functional outcomes; although estimates vary, based on uncontrolled studies, a high percentage of patients discontinued long-term opioid use because of lack of efficacy and because of adverse events (10).

The GRADE evidence summary with type of evidence ratings for the five clinical questions for the current evidence review are outlined (Table 1 ). This summary is based on studies included in the AHRQ 2014 review (35 studies) plus additional studies identified in the updated search (seven studies). Additional details on findings from the original review are provided in the full 2014 AHRQ report $(14,52)$. Full details on the clinical evidence review findings supporting this guideline are provided in the Clinical Evidence Review (http://stacks.cdc.gov/view/cdc/38026).

\section{Effectiveness}

For KQ1, no study of opioid therapy versus placebo, no opioid therapy, or nonopioid therapy for chronic pain evaluated long-term ( $\geq 1$ year) outcomes related to pain, function, or quality of life. Most placebo-controlled randomized clinical trials were $\leq 6$ weeks in duration. Thus, the body of evidence for KQ1 is rated as insufficient (0 studies contributing) (14).

\section{Harms}

For KQ2, the body of evidence is rated as type 3 (12 studies contributing; 11 from the original review plus one new study). One fair-quality cohort study found that long-term opioid therapy is associated with increased risk for an opioid abuse or dependence diagnosis (as defined by ICD-9-CM codes) versus no opioid prescription (22). Rates of opioid abuse or dependence diagnosis ranged from $0.7 \%$ with lower-dose ( $\leq 36 \mathrm{MME}$ ) chronic therapy to $6.1 \%$ with higher-dose ( $\geq 120 \mathrm{MME}$ ) chronic therapy, versus $0.004 \%$ with no opioids prescribed. Ten fair-quality uncontrolled studies reported estimates of opioid abuse, addiction, and related outcomes (5565). In primary care settings, prevalence of opioid dependence 
(using DSM-IV criteria) ranged from 3\% to $26 \%(55,56,59)$. In pain clinic settings, prevalence of addiction ranged from $2 \%$ to $14 \%(57,58,60,61,63-65)$.

Factors associated with increased risk for misuse included history of substance use disorder, younger age, major depression, and use of psychotropic medications $(55,62)$. Two studies reported on the association between opioid use and risk for overdose $(66,67)$. One large fair-quality retrospective cohort study found that recent opioid use was associated with increased risk for any overdose events and serious overdose events versus nonuse (66). It also found higher doses associated with increased risk. Relative to 1-19 MME/day, the adjusted hazard ratio (HR) for any overdose event (consisting of mostly nonfatal overdose) was 1.44 for 20 to $49 \mathrm{MME} /$ day, 3.73 for 50-99 MME/day, and 8.87 for $\geq 100 \mathrm{MME} /$ day. A similar pattern was observed for serious overdose. A good-quality population-based, nested case-control study also found a dose-dependent association with risk for overdose death (67). Relative to 1-19 MME/day, the adjusted odds ratio (OR) was 1.32 for 20-49 MME/day, 1.92 for 50-99 MME/day, 2.04 for 100-199 MME/day, and 2.88 for $\geq 200 \mathrm{MME} /$ day.

Findings of increased fracture risk for current opioid use, versus nonuse, were mixed in two studies $(68,69)$. Two studies found an association between opioid use and increased risk for cardiovascular events $(70,71)$. Indirect evidence was found for endocrinologic harms (increased use of medications for erectile dysfunction or testosterone from one previously included study; laboratory-defined androgen deficiency from one newly reviewed study) (72,73). One study found that opioid dosages $\geq 20 \mathrm{MME} /$ day were associated with increased odds of road trauma among drivers (74).

\section{Opioid Dosing Strategies}

For KQ3, the body of evidence is rated as type 4 (14 studies contributing; 12 from the original review plus two new studies). For initiation and titration of opioids, the 2014 AHRQ report found insufficient evidence from three fair-quality, open-label trials to determine comparative effectiveness of ER/LA versus immediate-release opioids for titrating patients to stable pain control $(75,76)$. One new fair-quality cohort study of Veterans Affairs patients found initiation of therapy with an ER/LA opioid associated with greater risk for nonfatal overdose than initiation with an immediate-release opioid, with risk greatest in the first 2 weeks after initiation of treatment (77).

For comparative effectiveness and harms of ER/LA opioids, the 2014 AHRQ report included three randomized, headto-head trials of various ER/LA opioids that found no clear differences in 1-year outcomes related to pain or function (78-80) but had methodological shortcomings. A fair-quality retrospective cohort study based on national Veterans Health
Administration system pharmacy data found that methadone was associated with lower overall risk for all-cause mortality versus morphine (81), and a fair-quality retrospective cohort study based on Oregon Medicaid data found no statistically significant differences between methadone and long-acting morphine in risk for death or overdose symptoms (82). However, a new observational study (83) found methadone associated with increased risk for overdose versus sustainedrelease morphine among Tennessee Medicaid patients. The observed inconsistency in study findings suggests that risks of methadone might vary in different settings as a function of different monitoring and management protocols, though more research is needed to understand factors associated with safer methadone prescribing.

For dose escalation, the 2014 AHRQ report included one fair-quality randomized trial that found no differences between more liberal dose escalation and maintenance of current doses after 12 months in pain, function, all-cause withdrawals, or withdrawals due to opioid misuse (84). However, the difference in opioid dosages prescribed at the end of the trial was relatively small (mean $52 \mathrm{MME} /$ day with more liberal dosing versus $40 \mathrm{MME} /$ day). Evidence on other comparisons related to opioid dosing strategies (ER/LA versus immediaterelease opioids; immediate-release plus ER/LA opioids versus ER/LA opioids alone; scheduled continuous dosing versus as-needed dosing; or opioid rotation versus maintenance of current therapy; long-term effects of strategies for treating acute exacerbations of chronic pain) was not available or too limited to determine effects on long-term clinical outcomes. For example, evidence on the comparative effectiveness of opioid tapering or discontinuation versus maintenance, and of different opioid tapering strategies, was limited to small, poor-quality studies (85-87).

\section{Risk Assessment and Mitigation}

For KQ4, the body of evidence is rated as type 3 for the accuracy of risk assessment tools and insufficient for the effectiveness of use of risk assessment tools and mitigation strategies in reducing harms (six studies contributing; four from the original review plus two new studies). The 2014 AHRQ report included four studies (88-91) on the accuracy of risk assessment instruments, administered prior to opioid therapy initiation, for predicting opioid abuse or misuse. Results for the Opioid Risk Tool (ORT) (89-91) were extremely inconsistent; evidence for other risk assessment instruments was very sparse, and studies had serious methodological shortcomings. One additional fair-quality (92) and one poor-quality (93) study identified for this update compared the predictive accuracy of the ORT, the Screener and Opioid Assessment for Patients with Pain-Revised (SOAPP-R), and the Brief Risk Interview. 
For the ORT, sensitivity was 0.58 and 0.75 and specificity 0.54 and 0.86 ; for the SOAPP-R, sensitivity was 0.53 and 0.25 and specificity 0.62 and 0.73 ; and for the Brief Risk Interview, sensitivity was 0.73 and 0.83 and specificity 0.43 and 0.88 . For the ORT, positive likelihood ratios ranged from noninformative (positive likelihood ratio close to 1) to moderately useful (positive likelihood ratio $>5$ ). The SOAPP-R was associated with noninformative likelihood ratios (estimates close to 1) in both studies.

No study evaluated the effectiveness of risk mitigation strategies (use of risk assessment instruments, opioid management plans, patient education, urine drug testing, use of PDMP data, use of monitoring instruments, more frequent monitoring intervals, pill counts, or use of abuse-deterrent formulations) for improving outcomes related to overdose, addiction, abuse, or misuse.

\section{Effects of Opioid Therapy for Acute Pain on Long-Term Use}

For KQ5, the body of evidence is rated as type 3 (two new studies contributing). Two fair-quality retrospective cohort studies found opioid therapy prescribed for acute pain associated with greater likelihood of long-term use. One study evaluated opioid-naïve patients who had undergone low-risk surgery, such as cataract surgery and varicose vein stripping (94). Use of opioids within 7 days of surgery was associated with increased risk for use at 1 year. The other study found that among patients with a workers' compensation claim for acute low back pain, compared to patients who did not receive opioids early after injury (defined as use within 15 days following onset of pain), patients who did receive early opioids had an increased likelihood of receiving five or more opioid prescriptions 30-730 days following onset that increased with greater early exposure. Versus no early opioid use, the adjusted OR was 2.08 (95\% CI = 1.55-2.78) for 1-140 MME/day and increased to $6.14(95 \%$ confidence interval $[\mathrm{CI}]=4.92-7.66)$ for $\geq 450 \mathrm{MME} /$ day (95).

\section{Summary of the Contextual Evidence Review}

\section{Primary Areas of Focus}

Contextual evidence is complementary information that assists in translating the clinical research findings into recommendations. CDC conducted contextual evidence reviews on four topics to supplement the clinical evidence review findings:
- Effectiveness of nonpharmacologic (e.g., cognitive behavioral therapy [CBT], exercise therapy, interventional treatments, and multimodal pain treatment) and nonopioid pharmacologic treatments (e.g., acetaminophen, nonsteroidal anti-inflammatory drugs [NSAIDs], antidepressants, and anticonvulsants), including studies of any duration.

- Benefits and harms of opioid therapy (including additional studies not included in the clinical evidence review, such as studies that were not restricted to patients with chronic pain, evaluated outcomes at any duration, performed ecological analyses, or used observational study designs other than cohort and case-cohort control studies) related to specific opioids, high-dose therapy, co-prescription with other controlled substances, duration of use, special populations, and potential usefulness of risk stratification/ mitigation approaches, in addition to effectiveness of treatments associated with addressing potential harms of opioid therapy (opioid use disorder).

- Clinician and patient values and preferences related to opioids and medication risks, benefits, and use.

- Resource allocation including costs and economic efficiency of opioid therapy and risk mitigation strategies.

$\mathrm{CDC}$ also reviewed clinical guidelines that were relevant to opioid prescribing and could inform or complement the CDC recommendations under development (e.g., guidelines on nonpharmacologic and nonopioid pharmacologic treatments and guidelines with recommendations related to specific clinician actions such as urine drug testing or opioid tapering protocols).

\section{Contextual Evidence Review Methods}

CDC conducted a contextual evidence review to assist in developing the recommendations by providing an assessment of the balance of benefits and harms, values and preferences, and cost, consistent with the GRADE approach. Given the public health urgency for developing opioid prescribing recommendations, a rapid review was required for the contextual evidence review for the current guideline. Rapid reviews are used when there is a need to streamline the systematic review process to obtain evidence quickly (96). Methods used to streamline the process include limiting searches by databases, years, and languages considered, and truncating quality assessment and data abstraction protocols. CDC conducted "rapid reviews" of the contextual evidence on nonpharmacologic and nonopioid pharmacologic treatments, benefits and harms, values and preferences, and resource allocation.

Detailed information about contextual evidence data sources and searches, inclusion criteria, study selection, and 
data extraction and synthesis are provided in the Contextual Evidence Review (http://stacks.cdc.gov/view/cdc/38027). In brief, CDC conducted systematic literature searches to identify original studies, systematic reviews, and clinical guidelines, depending on the topic being searched. CDC also solicited publication referrals from subject matter experts. Given the need for a rapid review process, grey literature (e.g., literature by academia, organizations, or government in the forms of reports, documents, or proceedings not published by commercial publishers) was not systematically searched. Database sources, including MEDLINE, PsycINFO, the Cochrane Central Register of Controlled Trials, and the Cochrane Database of Systematic Reviews, varied by topic. Multiple reviewers scanned study abstracts identified through the database searches and extracted relevant studies for review. CDC constructed narrative summaries and tables based on relevant articles that met inclusion criteria, which are provided in the Contextual Evidence Review (http://stacks.cdc.gov/ view/cdc/38027).

Findings from the contextual reviews provide indirect evidence and should be interpreted accordingly. CDC did not formally rate the quality of evidence for the studies included in the contextual evidence review using the GRADE method. The studies that addressed benefits and harms, values and preferences, and resource allocation most often employed observational methods, used short follow-up periods, and evaluated selected samples. Therefore the strength of the evidence from these contextual review areas was considered to be low, comparable to type 3 or type 4 evidence. The quality of evidence for nonopioid pharmacologic and nonpharmacologic pain treatments was generally rated as moderate, comparable to type 2 evidence, in systematic reviews and clinical guidelines (e.g., for treatment of chronic neuropathic pain, low back pain, osteoarthritis, and fibromyalgia). Similarly, the quality of evidence on pharmacologic and psychosocial opioid use disorder treatment was generally rated as moderate, comparable to type 2 evidence, in systematic reviews and clinical guidelines.

\section{Summary of Findings for Contextual Areas}

Full narrative reviews and tables that summarize key findings from the contextual evidence review are provided in the Contextual Evidence Review (http://stacks.cdc.gov/view/cdc/38027).

\section{Effectiveness of Nonpharmacologic and Nonopioid Pharmacologic Treatments}

Several nonpharmacologic and nonopioid pharmacologic treatments have been shown to be effective in managing chronic pain in studies ranging in duration from 2 weeks to 6 months. For example, CBT that trains patients in behavioral techniques and helps patients modify situational factors and cognitive processes that exacerbate pain has small positive effects on disability and catastrophic thinking (97). Exercise therapy can help reduce pain and improve function in chronic low back pain (98), improve function and reduce pain in osteoarthritis of the knee (99) and hip (100), and improve well-being, fibromyalgia symptoms, and physical function in fibromyalgia (101). Multimodal and multidisciplinary therapies (e.g., therapies that combine exercise and related therapies with psychologically based approaches) can help reduce pain and improve function more effectively than single modalities $(102,103)$. Nonopioid pharmacologic approaches used for pain include analgesics such as acetaminophen, NSAIDs, and cyclooxygenase 2 (COX-2) inhibitors; selected anticonvulsants; and selected antidepressants (particularly tricyclics and serotonin and norepinephrine reuptake inhibitors [SNRIs]). Multiple guidelines recommend acetaminophen as first-line pharmacotherapy for osteoarthritis (104-109) or for low back pain (110) but note that it should be avoided in liver failure and that dosage should be reduced in patients with hepatic insufficiency or a history of alcohol abuse (109). Although guidelines also recommend NSAIDs as first-line treatment for osteoarthritis or low back pain $(106,110)$, NSAIDs and COX-2 inhibitors do have risks, including gastrointestinal bleeding or perforation as well as renal and cardiovascular risks (111). FDA has recently strengthened existing label warnings that NSAIDs increase risks for heart attack and stroke, including that these risks might increase with longer use or at higher doses (112). Several guidelines agree that first- and second-line drugs for neuropathic pain include anticonvulsants (gabapentin or pregabalin), tricyclic antidepressants, and SNRIs (113-116). Interventional approaches such as epidural injection for certain conditions (e.g., lumbar radiculopathy) can provide short-term improvement in pain (117-119). Epidural injection has been associated with rare but serious adverse events, including loss of vision, stroke, paralysis, and death (120).

\section{Benefits and Harms of Opioid Therapy}

Balance between benefits and harms is a critical factor influencing the strength of clinical recommendations. In particular, CDC considered what is known from the epidemiology research about benefits and harms related to specific opioids and formulations, high dose therapy, co-prescription with other controlled substances, duration of use, special populations, and risk stratification and mitigation approaches. Additional information on benefits and harms of long-term opioid therapy from studies meeting rigorous selection criteria is provided in the clinical evidence review (e.g., see KQ2). CDC also considered the number of persons experiencing chronic pain, numbers potentially benefiting 
from opioids, and numbers affected by opioid-related harms. A review of these data is presented in the background section of this document, with detailed information provided in the Contextual Evidence Review (http://stacks.cdc.gov/view/ cdc/38027). Finally, CDC considered the effectiveness of treatments that addressed potential harms of opioid therapy (opioid use disorder).

Regarding specific opioids and formulations, as noted by FDA, there are serious risks of ER/LA opioids, and the indication for this class of medications is for management of pain severe enough to require daily, around-the-clock, longterm opioid treatment in patients for whom other treatment options (e.g., nonopioid analgesics or immediate-release opioids) are ineffective, not tolerated, or would be otherwise inadequate to provide sufficient management of pain (121). Time-scheduled opioid use was associated with substantially higher average daily opioid dosage than as-needed opioid use in one study (122). Methadone has been associated with disproportionate numbers of overdose deaths relative to the frequency with which it is prescribed for pain. Methadone has been found to account for as much as a third of opioidrelated overdose deaths involving single or multiple drugs in states that participated in the Drug Abuse Warning Network, which was more than any opioid other than oxycodone, despite representing $<2 \%$ of opioid prescriptions outside of opioid treatment programs in the United States; further, methadone was involved in twice as many single-drug deaths as any other prescription opioid (123).

Regarding high-dose therapy, several epidemiologic studies that were excluded from the clinical evidence review because patient samples were not restricted to patients with chronic pain also examined the association between opioid dosage and overdose risk (23,24,124-126). Consistent with the clinical evidence review, the contextual review found that opioid-related overdose risk is dosedependent, with higher opioid dosages associated with increased overdose risk. Two of these studies $(23,24)$, as well as the two studies in the clinical evidence review $(66,67)$, evaluated similar $\mathrm{MME} /$ day dose ranges for association with overdose risk. In these four studies, compared with opioids prescribed at <20 MME/ day, the odds of overdose among patients prescribed opioids for chronic nonmalignant pain were between 1.3 (67) and 1.9 (24) for dosages of 20 to $<50 \mathrm{MME} /$ day, between 1.9 (67) and 4.6 (24) for dosages of 50 to $<100 \mathrm{MME} /$ day, and between $2.0(67)$ and 8.9 (66) for dosages of $\geq 100 \mathrm{MME} /$ day. Compared with dosages of 1-<20 MME/day, absolute risk difference approximation for $50-<100 \mathrm{MME} /$ day was $0.15 \%$ for fatal overdose (24) and $1.40 \%$ for any overdose (66), and for $\geq 100 \mathrm{MME} /$ day was $0.25 \%$ for fatal overdose (24) and 4.04\% for any overdose (66). A recent study of Veterans Health Administration patients with chronic pain found that patients who died of overdoses related to opioids were prescribed higher opioid dosages (mean: $98 \mathrm{MME} /$ day; median: $60 \mathrm{MME} /$ day) than controls (mean: $48 \mathrm{MME} /$ day, median: $25 \mathrm{MME} /$ day) (127). Finally, another recent study of overdose deaths among state residents with and without opioid prescriptions revealed that prescription opioid-related overdose mortality rates rose rapidly up to prescribed doses of $200 \mathrm{MME} /$ day, after which the mortality rates continued to increase but grew more gradually (128). A listing of common opioid medications and their MME equivalents is provided (Table 2).

Regarding coprescription of opioids with benzodiazepines, epidemiologic studies suggest that concurrent use of benzodiazepines and opioids might put patients at greater risk for potentially fatal overdose. Three studies of fatal overdose deaths found evidence of concurrent benzodiazepine use in $31 \%-61 \%$ of decedents $(67,128,129)$. In one of these studies (67), among decedents who received an opioid prescription, those whose deaths were related to opioids were more likely to have obtained opioids from multiple physicians and pharmacies than decedents whose deaths were not related to opioids.

Regarding duration of use, patients can experience tolerance and loss of effectiveness of opioids over time (130). Patients who do not experience clinically meaningful pain relief early in treatment (i.e., within 1 month) are unlikely to experience pain relief with longer-term use (131).

Regarding populations potentially at greater risk for harm, risk is greater for patients with sleep apnea or other causes of sleep-disordered breathing, patients with renal or hepatic insufficiency, older adults, pregnant women, patients with depression or other mental health conditions, and patients with alcohol or other substance use disorders. Interpretation of clinical data on the effects of opioids on sleep-disordered breathing is difficult because of the types of study designs and methods employed, and there is no clear consensus regarding association with risk for developing obstructive sleep apnea syndrome (132). However, opioid therapy can decrease respiratory drive, a high percentage of patients on long-term opioid therapy have been reported to have an abnormal apneahypopnea index (133), opioid therapy can worsen central sleep apnea in obstructive sleep apnea patients, and it can cause further desaturation in obstructive sleep apnea patients not on continuous positive airway pressure (CPAP) (31). Reduced renal or hepatic function can result in greater peak effect and longer duration of action and reduce the dose at which respiratory depression and overdose occurs (134). Age-related changes in patients aged $\geq 65$ years, such as reduced renal function and medication clearance, even in the absence of renal disease (135), result in a smaller therapeutic window between safe dosages and dosages associated with respiratory depression and overdose. Older adults might also be at increased risk for falls and fractures related to opioids (136-138). Opioids used 
in pregnancy can be associated with additional risks to both mother and fetus. Some studies have shown an association of opioid use in pregnancy with birth defects, including neural tube defects $(139,140)$, congenital heart defects $(140)$, and gastroschisis $(140)$; preterm delivery (141), poor fetal growth (141), and stillbirth (141). Importantly, in some cases, opioid use during pregnancy leads to neonatal opioid withdrawal syndrome (142). Patients with mental health comorbidities and patients with histories of substance use disorders might be at higher risk than other patients for opioid use disorder $(62,143,144)$. Recent analyses found that depressed patients were at higher risk for drug overdose than patients without depression, particularly at higher opioid dosages, although investigators were unable to distinguish unintentional overdose from suicide attempts (145). In case-control and case-cohort studies, substance abuse/dependence was more prevalent among patients experiencing overdose than among patients not experiencing overdose (12\% versus $6 \%$ [66, $40 \%$ versus $10 \%$ [24], and $26 \%$ versus $9 \%$ [23]).

Regarding risk stratification approaches, limited evidence was found regarding benefits and harms. Potential benefits of PDMPs and urine drug testing include the ability to identify patients who might be at higher risk for opioid overdose or opioid use disorder, and help determine which patients will benefit from greater caution and increased monitoring or interventions when risk factors are present. For example, one study found that most fatal overdoses could be identified retrospectively on the basis of two pieces of information, multiple prescribers and high total daily opioid dosage, both important risk factors for overdose $(124,146)$ that are available to prescribers in the PDMP (124). However, limited evaluation of PDMPs at the state level has revealed mixed effects on changes in prescribing and mortality outcomes (28). Potential harms of risk stratification include underestimation of risks of opioid therapy when screening tools are not adequately sensitive, as well as potential overestimation of risk, which could lead to inappropriate clinical decisions.

Regarding risk mitigation approaches, limited evidence was found regarding benefits and harms. Although no studies were found to examine prescribing of naloxone with opioid pain medication in primary care settings, naloxone distribution through community-based programs providing prevention services for substance users has been demonstrated to be associated with decreased risk for opioid overdose death at the community level (147).

Concerns have been raised that prescribing changes such as dose reduction might be associated with unintended negative consequences, such as patients seeking heroin or other illicitly obtained opioids (148) or interference with appropriate pain treatment (149). With the exception of a study noting an association between an abuse-deterrent formulation of OxyContin and heroin use, showing that some patients in qualitative interviews reported switching to another opioid, including heroin, for many reasons, including cost and availability as well as ease of use (150), CDC did not identify studies evaluating these potential outcomes.

Finally, regarding the effectiveness of opioid use disorder treatments, methadone and buprenorphine for opioid use disorder have been found to increase retention in treatment and to decrease illicit opioid use among patients with opioid use disorder involving heroin (151-153). Although findings are mixed, some studies suggest that effectiveness is enhanced when psychosocial treatments (e.g., contingency management, community reinforcement, psychotherapeutic counseling, and family therapy) are used in conjunction with medicationassisted therapy; for example, by reducing opioid misuse and increasing retention during maintenance therapy, and improving compliance after detoxification $(154,155)$.

\section{Clinician and Patient Values and Preferences}

Clinician and patient values and preferences can inform how benefits and harms of long-term opioid therapy are weighted and estimate the effort and resources required to effectively provide implementation support. Many physicians lack confidence in their ability to prescribe opioids safely (156), to predict (157) or detect (158) prescription drug abuse, and to discuss abuse with their patients (158). Although clinicians have reported favorable beliefs and attitudes about improvements in pain and quality of life attributed to opioids (159), most consider prescription drug abuse to be a "moderate" or "big" problem in their community, and large proportions are "very" concerned about opioid addiction (55\%) and death (48\%) (160). Clinicians do not consistently use practices intended to decrease the risk for misuse, such as PDMPs $(161,162)$, urine drug testing (163), and opioid treatment agreements (164). This is likely due in part to challenges related to registering for PDMP access and logging into the PDMP (which can interrupt normal clinical workflow if data are not integrated into electronic health record systems) (165), competing clinical demands, perceived inadequate time to discuss the rationale for urine drug testing and to order confirmatory testing, and feeling unprepared to interpret and address results (166).

Many patients do not have an opinion about "opioids" or know what this term means (167). Most are familiar with the term "narcotics." About a third associated "narcotics" with addiction or abuse, and about half feared "addiction" from long-term "narcotic" use (168). Most patients taking opioids experience side effects $(73 \%$ of patients taking hydrocodone for noncancer pain [11], 96\% of patients taking opioids for chronic pain [12]), and side effects, rather than pain relief, 
have been found to explain most of the variation in patients' preferences related to taking opioids (12). For example, patients taking hydrocodone for noncancer pain commonly reported side effects including dizziness, headache, fatigue, drowsiness, nausea, vomiting, and constipation (11). Patients with chronic pain in focus groups emphasized effectiveness of goal setting for increasing motivation and functioning (168). Patients taking high dosages report reliance on opioids despite ambivalence about their benefits (169) and regardless of pain reduction, reported problems, concerns, side effects, or perceived helpfulness (13).

\section{Resource Allocation}

Resource allocation (cost) is an important consideration in understanding the feasibility of clinical recommendations. CDC searched for evidence on opioid therapy compared with other treatments; costs of misuse, abuse, and overdose from prescription opioids; and costs of specific risk mitigation strategies (e.g., urine drug testing). Yearly direct and indirect costs related to prescription opioids have been estimated (based on studies published since 2010) to be $\$ 53.4$ billion for nonmedical use of prescription opioids (170); $\$ 55.7$ billion for abuse, dependence (i.e., opioid use disorder), and misuse of prescription opioids (171); and $\$ 20.4$ billion for direct and indirect costs related to opioid-related overdose alone (172). In 2012, total expenses for outpatient prescription opioids were estimated at $\$ 9.0$ billion, an increase of $120 \%$ from 2002 (173). Although there are perceptions that opioid therapy for chronic pain is less expensive than more timeintensive nonpharmacologic management approaches, many pain treatments, including acetaminophen, NSAIDs, tricyclic antidepressants, and massage therapy, are associated with lower mean and median annual costs compared with opioid therapy (174). COX-2 inhibitors, SNRIs, anticonvulsants, topical analgesics, physical therapy, and CBT are also associated with lower median annual costs compared with opioid therapy (174). Limited information was found on costs of strategies to decrease risks associated with opioid therapy; however, urine drug testing, including screening and confirmatory tests, has been estimated to cost $\$ 211-\$ 363$ per test (175).

\section{Recommendations}

The recommendations are grouped into three areas for consideration:

- Determining when to initiate or continue opioids for chronic pain.

- Opioid selection, dosage, duration, follow-up, and discontinuation.

- Assessing risk and addressing harms of opioid use.
There are 12 recommendations (Box 1). Each recommendation is followed by a rationale for the recommendation, with considerations for implementation noted. In accordance with the ACIP GRADE process, CDC based the recommendations on consideration of the clinical evidence, contextual evidence (including benefits and harms, values and preferences, resource allocation), and expert opinion. For each recommendation statement, $\mathrm{CDC}$ notes the recommendation category (A or B) and the type of the evidence $(1,2,3$, or 4$)$ supporting the statement (Box 2). Expert opinion is reflected within each of the recommendation rationales. While there was not an attempt to reach consensus among experts, experts from the Core Expert Group and from the Opioid Guideline Workgroup ("experts") expressed overall, general support for all recommendations. Where differences in expert opinion emerged for detailed actions within the clinical recommendations or for implementation considerations, CDC notes the differences of opinion in the supporting rationale statements.

Category A recommendations indicate that most patients should receive the recommended course of action; category $\mathrm{B}$ recommendations indicate that different choices will be appropriate for different patients, requiring clinicians to help patients arrive at a decision consistent with patient values and preferences and specific clinical situations. Consistent with the ACIP (47) and GRADE process (48), category A recommendations were made, even with type 3 and 4 evidence, when there was broad agreement that the advantages of a clinical action greatly outweighed the disadvantages based on a consideration of benefits and harms, values and preferences, and resource allocation. Category $\mathrm{B}$ recommendations were made when there was broad agreement that the advantages and disadvantages of a clinical action were more balanced, but advantages were significant enough to warrant a recommendation. All recommendations are category $\mathrm{A}$ recommendations, with the exception of recommendation 10 , which is rated as category B. Recommendations were associated with a range of evidence types, from type 2 to type 4 .

In summary, the categorization of recommendations was based on the following assessment:

- No evidence shows a long-term benefit of opioids in pain and function versus no opioids for chronic pain with outcomes examined at least 1 year later (with most placebocontrolled randomized trials $\leq 6$ weeks in duration).

- Extensive evidence shows the possible harms of opioids (including opioid use disorder, overdose, and motor vehicle injury).

- Extensive evidence suggests some benefits of nonpharmacologic and nonopioid pharmacologic treatments compared with long-term opioid therapy, with less harm. 


\section{Determining When to Initiate or Continue Opioids for Chronic Pain}

1. Nonpharmacologic therapy and nonopioid pharmacologic therapy are preferred for chronic pain. Clinicians should consider opioid therapy only if expected benefits for both pain and function are anticipated to outweigh risks to the patient. If opioids are used, they should be combined with nonpharmacologic therapy and nonopioid pharmacologic therapy, as appropriate.

2. Before starting opioid therapy for chronic pain, clinicians should establish treatment goals with all patients, including realistic goals for pain and function, and should consider how therapy will be discontinued if benefits do not outweigh risks. Clinicians should continue opioid therapy only if there is clinically meaningful improvement in pain and function that outweighs risks to patient safety.

3. Before starting and periodically during opioid therapy, clinicians should discuss with patients known risks and realistic benefits of opioid therapy and patient and clinician responsibilities for managing therapy.

\section{Opioid Selection, Dosage, Duration, Follow-Up, and Discontinuation}

4. When starting opioid therapy for chronic pain, clinicians should prescribe immediate-release opioids instead of extended-release/long-acting (ER/LA) opioids.

5. When opioids are started, clinicians should prescribe the lowest effective dosage. Clinicians should use caution when prescribing opioids at any dosage, should carefully reassess evidence of individual benefits and risks when increasing dosage to $\geq 50$ morphine milligram equivalents (MME)/day, and should avoid increasing dosage to $\geq 90 \mathrm{MME} /$ day or carefully justify a decision to titrate dosage to $\geq 90 \mathrm{MME} /$ day.

6. Long-term opioid use often begins with treatment of acute pain. When opioids are used for acute pain, clinicians should prescribe the lowest effective dose of immediate-release opioids and should prescribe no greater quantity than needed for the expected duration of pain severe enough to require opioids. Three days or less will often be sufficient; more than seven days will rarely be needed.
7. Clinicians should evaluate benefits and harms with patients within 1 to 4 weeks of starting opioid therapy for chronic pain or of dose escalation. Clinicians should evaluate benefits and harms of continued therapy with patients every 3 months or more frequently. If benefits do not outweigh harms of continued opioid therapy, clinicians should optimize other therapies and work with patients to taper opioids to lower dosages or to taper and discontinue opioids.

\section{Assessing Risk and Addressing Harms of Opioid Use}

8. Before starting and periodically during continuation of opioid therapy, clinicians should evaluate risk factors for opioid-related harms. Clinicians should incorporate into the management plan strategies to mitigate risk, including considering offering naloxone when factors that increase risk for opioid overdose, such as history of overdose, history of substance use disorder, higher opioid dosages ( $\geq 50 \mathrm{MME} /$ day), or concurrent benzodiazepine use, are present.

9. Clinicians should review the patient's history of controlled substance prescriptions using state prescription drug monitoring program (PDMP) data to determine whether the patient is receiving opioid dosages or dangerous combinations that put him or her at high risk for overdose. Clinicians should review PDMP data when starting opioid therapy for chronic pain and periodically during opioid therapy for chronic pain, ranging from every prescription to every 3 months.

10. When prescribing opioids for chronic pain, clinicians should use urine drug testing before starting opioid therapy and consider urine drug testing at least annually to assess for prescribed medications as well as other controlled prescription drugs and illicit drugs.

11. Clinicians should avoid prescribing opioid pain medication and benzodiazepines concurrently whenever possible.

12. Clinicians should offer or arrange evidence-based treatment (usually medication-assisted treatment with buprenorphine or methadone in combination with behavioral therapies) for patients with opioid use disorder.

*All recommendations are category A (apply to all patients outside of active cancer treatment, palliative care, and end-of-life care) except recommendation 10 (designated category $\mathrm{B}$, with individual decision making required); see full guideline for evidence ratings. 
BOX 2. Interpretation of recommendation categories and evidence type

\section{Recommendation Categories}

Based on evidence type, balance between desirable and undesirable effects, values and preferences, and resource allocation (cost).

Category A recommendation: Applies to all persons; most patients should receive the recommended course of action.

Category B recommendation: Individual decision making needed; different choices will be appropriate for different patients. Clinicians help patients arrive at a decision consistent with patient values and preferences and specific clinical situations.

\section{Evidence Type}

Based on study design as well as a function of limitations in study design or implementation, imprecision of estimates, variability in findings, indirectness of evidence, publication bias, magnitude of treatment effects, doseresponse gradient, and constellation of plausible biases that could change effects.

Type 1 evidence: Randomized clinical trials or overwhelming evidence from observational studies.

Type 2 evidence: Randomized clinical trials with important limitations, or exceptionally strong evidence from observational studies.

Type 3 evidence: Observational studies or randomized clinical trials with notable limitations.

Type 4 evidence: Clinical experience and observations, observational studies with important limitations, or randomized clinical trials with several major limitations.

\section{Determining When to Initiate or Continue Opioids for Chronic Pain}

1. Nonpharmacologic therapy and nonopioid pharmacologic therapy are preferred for chronic pain. Clinicians should consider opioid therapy only if expected benefits for both pain and function are anticipated to outweigh risks to the patient. If opioids are used, they should be combined with nonpharmacologic therapy and nonopioid pharmacologic therapy, as appropriate (recommendation category: A, evidence type: 3 ).

Patients with pain should receive treatment that provides the greatest benefits relative to risks. The contextual evidence review found that many nonpharmacologic therapies, including physical therapy, weight loss for knee osteoarthritis, psychological therapies such as CBT, and certain interventional procedures can ameliorate chronic pain. There is high-quality evidence that exercise therapy (a prominent modality in physical therapy) for hip (100) or knee (99) osteoarthritis reduces pain and improves function immediately after treatment and that the improvements are sustained for at least 2-6 months. Previous guidelines have strongly recommended aerobic, aquatic, and/or resistance exercises for patients with osteoarthritis of the knee or hip (176). Exercise therapy also can help reduce pain and improve function in low back pain and can improve global well-being and physical function in fibromyalgia $(98,101)$. Multimodal therapies and multidisciplinary biopsychosocial rehabilitation-combining approaches (e.g., psychological therapies with exercise) can reduce long-term pain and disability compared with usual care and compared with physical treatments (e.g., exercise) alone. Multimodal therapies are not always available or reimbursed by insurance and can be time-consuming and costly for patients. Interventional approaches such as arthrocentesis and intraarticular glucocorticoid injection for pain associated with rheumatoid arthritis (117) or osteoarthritis (118) and subacromial corticosteroid injection for rotator cuff disease (119) can provide short-term improvement in pain and function. Evidence is insufficient to determine the extent to which repeated glucocorticoid injection increases potential risks such as articular cartilage changes (in osteoarthritis) and sepsis (118). Serious adverse events are rare but have been reported with epidural injection (120).

Several nonopioid pharmacologic therapies (including acetaminophen, NSAIDs, and selected antidepressants and anticonvulsants) are effective for chronic pain. In particular, acetaminophen and NSAIDs can be useful for arthritis and low back pain. Selected anticonvulsants such as pregabalin and gabapentin can improve pain in diabetic neuropathy and post-herpetic neuralgia (contextual evidence review). Pregabalin, gabapentin, and carbamazepine are FDA-approved for treatment of certain neuropathic pain conditions, and pregabalin is FDA approved for fibromyalgia management. In patients with or without depression, tricyclic antidepressants and SNRIs provide effective analgesia for neuropathic pain conditions including diabetic neuropathy and post-herpetic neuralgia, often at lower dosages and with a shorter time to onset of effect than for treatment of depression (see contextual evidence review). Tricyclics and SNRIs can also relieve fibromyalgia symptoms. The SNRI duloxetine is FDA-approved for the treatment of diabetic neuropathy and fibromyalgia. Because patients with chronic pain often suffer from concurrent depression (144), and depression can exacerbate physical symptoms including pain (177), patients with co-occurring pain and depression are especially likely to benefit from antidepressant medication (see Recommendation 8). Nonopioid pharmacologic therapies 
are not generally associated with substance use disorder, and the numbers of fatal overdoses associated with nonopioid medications are a fraction of those associated with opioid medications (contextual evidence review). For example, acetaminophen, NSAIDs, and opioid pain medication were involved in 881, 228, and 16,651 pharmaceutical overdose deaths in the United States in 2010 (178). However, nonopioid pharmacologic therapies are associated with certain risks, particularly in older patients, pregnant patients, and patients with certain co-morbidities such as cardiovascular, renal, gastrointestinal, and liver disease (see contextual evidence review). For example, acetaminophen can be hepatotoxic at dosages of $>3-4$ grams/day and at lower dosages in patients with chronic alcohol use or liver disease (109). NSAID use has been associated with gastritis, peptic ulcer disease, cardiovascular events $(111,112)$, and fluid retention, and most NSAIDs (choline magnesium trilisate and selective COX-2 inhibitors are exceptions) interfere with platelet aggregation (179). Clinicians should review FDA-approved labeling including boxed warnings before initiating treatment with any pharmacologic therapy.

Although opioids can reduce pain during short-term use, the clinical evidence review found insufficient evidence to determine whether pain relief is sustained and whether function or quality of life improves with long-term opioid therapy (KQ1). While benefits for pain relief, function, and quality of life with long-term opioid use for chronic pain are uncertain, risks associated with long-term opioid use are clearer and significant. Based on the clinical evidence review, long-term opioid use for chronic pain is associated with serious risks including increased risk for opioid use disorder, overdose, myocardial infarction, and motor vehicle injury (KQ2). At a population level, more than 165,000 persons in the United States have died from opioid pain-medication-related overdoses since 1999 (see Contextual Evidence Review).

Integrated pain management requires coordination of medical, psychological, and social aspects of health care and includes primary care, mental health care, and specialist services when needed (180). Nonpharmacologic physical and psychological treatments such as exercise and CBT are approaches that encourage active patient participation in the care plan, address the effects of pain in the patient's life, and can result in sustained improvements in pain and function without apparent risks. Despite this, these therapies are not always or fully covered by insurance, and access and cost can be barriers for patients. For many patients, aspects of these approaches can be used even when there is limited access to specialty care. For example, previous guidelines have strongly recommended aerobic, aquatic, and/or resistance exercises for patients with osteoarthritis of the knee or hip (176) and maintenance of activity for patients with low back pain (110). A randomized trial found no difference in reduced chronic low back pain intensity, frequency or disability between patients assigned to relatively low-cost group aerobics and individual physiotherapy or muscle reconditioning sessions (181). Low-cost options to integrate exercise include brisk walking in public spaces or use of public recreation facilities for group exercise. CBT addresses psychosocial contributors to pain and improves function (97). Primary care clinicians can integrate elements of a cognitive behavioral approach into their practice by encouraging patients to take an active role in the care plan, by supporting patients in engaging in beneficial but potentially anxiety-provoking activities, such as exercise (179), or by providing education in relaxation techniques and coping strategies. In many locations, there are free or low-cost patient support, self-help, and educational community-based programs that can provide stress reduction and other mental health benefits. Patients with more entrenched anxiety or fear related to pain, or other significant psychological distress, can be referred for formal therapy with a mental health specialist (e.g., psychologist, psychiatrist, clinical social worker). Multimodal therapies should be considered for patients not responding to single-modality therapy, and combinations should be tailored depending on patient needs, cost, and convenience.

To guide patient-specific selection of therapy, clinicians should evaluate patients and establish or confirm the diagnosis. Detailed recommendations on diagnosis are provided in other guidelines $(110,179)$, but evaluation should generally include a focused history, including history and characteristics of pain and potentially contributing factors (e.g., function, psychosocial stressors, sleep) and physical exam, with imaging or other diagnostic testing only if indicated (e.g., if severe or progressive neurologic deficits are present or if serious underlying conditions are suspected) $(110,179)$. For complex pain syndromes, pain specialty consultation can be considered to assist with diagnosis as well as management. Diagnosis can help identify disease-specific interventions to reverse or ameliorate pain; for example, improving glucose control to prevent progression of diabetic neuropathy; immune-modulating agents for rheumatoid arthritis; physical or occupational therapy to address posture, muscle weakness, or repetitive occupational motions that contribute to musculoskeletal pain; or surgical intervention to relieve mechanical/compressive pain (179). The underlying mechanism for most pain syndromes can be categorized as neuropathic (e.g., diabetic neuropathy, postherpetic neuralgia, fibromyalgia), or nociceptive (e.g., osteoarthritis, muscular back pain). The diagnosis and pathophysiologic mechanism of pain have implications for symptomatic pain treatment with medication. For example, evidence is limited or insufficient 
for improved pain or function with long-term use of opioids for several chronic pain conditions for which opioids are commonly prescribed, such as low back pain (182), headache (183), and fibromyalgia (184). Although NSAIDs can be used for exacerbations of nociceptive pain, other medications (e.g., tricyclics, selected anticonvulsants, or transdermal lidocaine) generally are recommended for neuropathic pain. In addition, improvement of neuropathic pain can begin weeks or longer after symptomatic treatment is initiated (179). Medications should be used only after assessment and determination that expected benefits outweigh risks given patient-specific factors. For example, clinicians should consider falls risk when selecting and dosing potentially sedating medications such as tricyclics, anticonvulsants, or opioids, and should weigh risks and benefits of use, dose, and duration of NSAIDs when treating older adults as well as patients with hypertension, renal insufficiency, or heart failure, or those with risk for peptic ulcer disease or cardiovascular disease. Some guidelines recommend topical NSAIDs for localized osteoarthritis (e.g., knee osteoarthritis) over oral NSAIDs in patients aged $\geq 75$ years to minimize systemic effects (176).

Experts agreed that opioids should not be considered firstline or routine therapy for chronic pain (i.e., pain continuing or expected to continue $>3$ months or past the time of normal tissue healing) outside of active cancer, palliative, and endof-life care, given small to moderate short-term benefits, uncertain long-term benefits, and potential for serious harms; although evidence on long-term benefits of nonopioid therapies is also limited, these therapies are also associated with short-term benefits, and risks are much lower. This does not mean that patients should be required to sequentially "fail" nonpharmacologic and nonopioid pharmacologic therapy before proceeding to opioid therapy. Rather, expected benefits specific to the clinical context should be weighed against risks before initiating therapy. In some clinical contexts (e.g., headache or fibromyalgia), expected benefits of initiating opioids are unlikely to outweigh risks regardless of previous nonpharmacologic and nonopioid pharmacologic therapies used. In other situations (e.g., serious illness in a patient with poor prognosis for return to previous level of function, contraindications to other therapies, and clinician and patient agreement that the overriding goal is patient comfort), opioids might be appropriate regardless of previous therapies used. In addition, when opioid pain medication is used, it is more likely to be effective if integrated with nonpharmacologic therapy. Nonpharmacologic approaches such as exercise and CBT should be used to reduce pain and improve function in patients with chronic pain. Nonopioid pharmacologic therapy should be used when benefits outweigh risks and should be combined with nonpharmacologic therapy to reduce pain and improve function. If opioids are used, they should be combined with nonpharmacologic therapy and nonopioid pharmacologic therapy, as appropriate, to provide greater benefits to patients in improving pain and function.

2. Before starting opioid therapy for chronic pain, clinicians should establish treatment goals with all patients, including realistic goals for pain and function, and should consider how opioid therapy will be discontinued if benefits do not outweigh risks. Clinicians should continue opioid therapy only if there is clinically meaningful improvement in pain and function that outweighs risks to patient safety (recommendation category: A, evidence type: 4).

The clinical evidence review found insufficient evidence to determine long-term benefits of opioid therapy for chronic pain and found an increased risk for serious harms related to long-term opioid therapy that appears to be dose-dependent. In addition, studies on currently available risk assessment instruments were sparse and showed inconsistent results (KQ4). The clinical evidence review for the current guideline considered studies with outcomes examined at $\geq 1$ year that compared opioid use versus nonuse or placebo. Studies of opioid therapy for chronic pain that did not have a nonopioid control group have found that although many patients discontinue opioid therapy for chronic noncancer pain due to adverse effects or insufficient pain relief, there is weak evidence that patients who are able to continue opioid therapy for at least 6 months can experience clinically significant pain relief and insufficient evidence that function or quality of life improves (185). These findings suggest that it is very difficult for clinicians to predict whether benefits of opioids for chronic pain will outweigh risks of ongoing treatment for individual patients. Opioid therapy should not be initiated without consideration of an "exit strategy" to be used if the therapy is unsuccessful.

Experts agreed that before opioid therapy is initiated for chronic pain outside of active cancer, palliative, and end-oflife care, clinicians should determine how effectiveness will be evaluated and should establish treatment goals with patients. Because the line between acute pain and initial chronic pain is not always clear, it might be difficult for clinicians to determine when they are initiating opioids for chronic pain rather than treating acute pain. Pain lasting longer than 3 months or past the time of normal tissue healing (which could be substantially shorter than 3 months, depending on the condition) is generally no longer considered acute. However, establishing treatment goals with a patient who has already received opioid therapy for 3 months would defer this discussion well past the point of 
initiation of opioid therapy for chronic pain. Clinicians often write prescriptions for long-term use in 30-day increments, and opioid prescriptions written for $\geq 30$ days are likely to represent initiation or continuation of long-term opioid therapy. Before writing an opioid prescription for $\geq 30$ days, clinicians should establish treatment goals with patients. Clinicians seeing new patients already receiving opioids should establish treatment goals for continued opioid therapy. Although the clinical evidence review did not find studies evaluating the effectiveness of written agreements or treatment plans (KQ4), clinicians and patients who set a plan in advance will clarify expectations regarding how opioids will be prescribed and monitored, as well as situations in which opioids will be discontinued or doses tapered (e.g., if treatment goals are not met, opioids are no longer needed, or adverse events put the patient at risk) to improve patient safety.

Experts thought that goals should include improvement in both pain relief and function (and therefore in quality of life). However, there are some clinical circumstances under which reductions in pain without improvement in physical function might be a more realistic goal (e.g., diseases typically associated with progressive functional impairment or catastrophic injuries such as spinal cord trauma). Experts noted that function can include emotional and social as well as physical dimensions. In addition, experts emphasized that mood has important interactions with pain and function. Experts agreed that clinicians may use validated instruments such as the threeitem "Pain average, interference with Enjoyment of life, and interference with General activity" (PEG) Assessment Scale (186) to track patient outcomes. Clinically meaningful improvement has been defined as a $30 \%$ improvement in scores for both pain and function (187). Monitoring progress toward patient-centered functional goals (e.g., walking the dog or walking around the block, returning to part-time work, attending family sports or recreational activities) can also contribute to the assessment of functional improvement. Clinicians should use these goals in assessing benefits of opioid therapy for individual patients and in weighing benefits against risks of continued opioid therapy (see Recommendation 7, including recommended intervals for follow-up). Because depression, anxiety, and other psychological co-morbidities often coexist with and can interfere with resolution of pain, clinicians should use validated instruments to assess for these conditions (see Recommendation 8) and ensure that treatment for these conditions is optimized. If patients receiving opioid therapy for chronic pain do not experience meaningful improvements in both pain and function compared with prior to initiation of opioid therapy, clinicians should consider working with patients to taper and discontinue opioids (see Recommendation 7) and should use nonpharmacologic and nonopioid pharmacologic approaches to pain management (see Recommendation 1).

\section{Before starting and periodically during opioid therapy, clinicians should discuss with patients known risks and realistic benefits of opioid therapy and patient and clinician responsibilities for managing therapy (recommendation category: A, evidence type: 3 ).}

The clinical evidence review did not find studies evaluating effectiveness of patient education or opioid treatment plans as risk-mitigation strategies (KQ4). However, the contextual evidence review found that many patients lack information about opioids and identified concerns that some clinicians miss opportunities to effectively communicate about safety. Given the substantial evidence gaps on opioids, uncertain benefits of long-term use, and potential for serious harms, patient education and discussion before starting opioid therapy are critical so that patient preferences and values can be understood and used to inform clinical decisions. Experts agreed that essential elements to communicate to patients before starting and periodically during opioid therapy include realistic expected benefits, common and serious harms, and expectations for clinician and patient responsibilities to mitigate risks of opioid therapy.

Clinicians should involve patients in decisions about whether to start or continue opioid therapy. Given potentially serious risks of long-term opioid therapy, clinicians should ensure that patients are aware of potential benefits of, harms of, and alternatives to opioids before starting or continuing opioid therapy. Clinicians are encouraged to have open and honest discussions with patients to inform mutual decisions about whether to start or continue opioid therapy. Important considerations include the following:

- Be explicit and realistic about expected benefits of opioids, explaining that while opioids can reduce pain during shortterm use, there is no good evidence that opioids improve pain or function with long-term use, and that complete relief of pain is unlikely (clinical evidence review, KQ1).

- Emphasize improvement in function as a primary goal and that function can improve even when pain is still present.

- Advise patients about serious adverse effects of opioids, including potentially fatal respiratory depression and development of a potentially serious lifelong opioid use disorder that can cause distress and inability to fulfill major role obligations.

- Advise patients about common effects of opioids, such as constipation, dry mouth, nausea, vomiting, drowsiness, confusion, tolerance, physical dependence, and withdrawal symptoms when stopping opioids. To prevent constipation associated with opioid use, advise patients to increase 
hydration and fiber intake and to maintain or increase physical activity. Stool softeners or laxatives might be needed.

- Discuss effects that opioids might have on ability to safely operate a vehicle, particularly when opioids are initiated, when dosages are increased, or when other central nervous system depressants, such as benzodiazepines or alcohol, are used concurrently.

- Discuss increased risks for opioid use disorder, respiratory depression, and death at higher dosages, along with the importance of taking only the amount of opioids prescribed, i.e., not taking more opioids or taking them more often.

- Review increased risks for respiratory depression when opioids are taken with benzodiazepines, other sedatives, alcohol, illicit drugs such as heroin, or other opioids.

- Discuss risks to household members and other individuals if opioids are intentionally or unintentionally shared with others for whom they are not prescribed, including the possibility that others might experience overdose at the same or at lower dosage than prescribed for the patient, and that young children are susceptible to unintentional ingestion. Discuss storage of opioids in a secure, preferably locked location and options for safe disposal of unused opioids (188).

- Discuss the importance of periodic reassessment to ensure that opioids are helping to meet patient goals and to allow opportunities for opioid discontinuation and consideration of additional nonpharmacologic or nonopioid pharmacologic treatment options if opioids are not effective or are harmful.

- Discuss planned use of precautions to reduce risks, including use of prescription drug monitoring program information (see Recommendation 9) and urine drug testing (see Recommendation 10). Consider including discussion of naloxone use for overdose reversal (see Recommendation 8).

- Consider whether cognitive limitations might interfere with management of opioid therapy (for older adults in particular) and, if so, determine whether a caregiver can responsibly co-manage medication therapy. Discuss the importance of reassessing safer medication use with both the patient and caregiver.

Given the possibility that benefits of opioid therapy might diminish or that risks might become more prominent over time, it is important that clinicians review expected benefits and risks of continued opioid therapy with patients periodically, at least every 3 months (see Recommendation 7).

\section{Opioid Selection, Dosage, Duration, Follow-Up, and Discontinuation}

\section{When starting opioid therapy for chronic pain, clinicians should prescribe immediate-release opioids instead of extended-release/long-acting (ER/LA) opioids (recommendation category: A, evidence type: 4).}

ER/LA opioids include methadone, transdermal fentanyl, and extended-release versions of opioids such as oxycodone, oxymorphone, hydrocodone, and morphine. The clinical evidence review found a fair-quality study showing a higher risk for overdose among patients initiating treatment with ER/LA opioids than among those initiating treatment with immediate-release opioids (77). The clinical evidence review did not find evidence that continuous, time-scheduled use of ER/LA opioids is more effective or safer than intermittent use of immediate-release opioids or that time-scheduled use of ER/ LA opioids reduces risks for opioid misuse or addiction (KQ3).

In 2014, the FDA modified the labeling for ER/LA opioid pain medications, noting serious risks and recommending that ER/LA opioids be reserved for "management of pain severe enough to require daily, around-the-clock, long-term opioid treatment" when "alternative treatment options (e.g., nonopioid analgesics or immediate-release opioids) are ineffective, not tolerated, or would be otherwise inadequate to provide sufficient management of pain" and not used as "as needed" pain relievers (121). FDA has also noted that some ER/LA opioids are only appropriate for opioid-tolerant patients, defined as patients who have received certain dosages of opioids (e.g., $60 \mathrm{mg}$ daily of oral morphine, $30 \mathrm{mg}$ daily of oral oxycodone, or equianalgesic dosages of other opioids) for at least 1 week (189). Time-scheduled opioid use can be associated with greater total average daily opioid dosage compared with intermittent, as-needed opioid use (contextual evidence review). In addition, experts indicated that there was not enough evidence to determine the safety of using immediate-release opioids for breakthrough pain when ER/ LA opioids are used for chronic pain outside of active cancer pain, palliative care, or end-of-life care, and that this practice might be associated with dose escalation.

Abuse-deterrent technologies have been employed to prevent manipulation intended to defeat extended-release properties of ER/LA opioids and to prevent opioid use by unintended routes of administration, such as injection of oral opioids. As indicated in FDA guidance for industry on evaluation and labeling of abuse-deterrent opioids (190), although abusedeterrent technologies are expected to make manipulation of opioids more difficult or less rewarding, they do not prevent 
opioid abuse through oral intake, the most common route of opioid abuse, and can still be abused by nonoral routes. The "abuse-deterrent" label does not indicate that there is no risk for abuse. No studies were found in the clinical evidence review assessing the effectiveness of abuse-deterrent technologies as a risk mitigation strategy for deterring or preventing abuse. In addition, abuse-deterrent technologies do not prevent unintentional overdose through oral intake. Experts agreed that recommendations could not be offered at this time related to use of abuse-deterrent formulations.

In comparing different ER/LA formulations, the clinical evidence review found inconsistent results for overdose risk with methadone versus other ER/LA opioids used for chronic pain (KQ3). The contextual evidence review found that methadone has been associated with disproportionate numbers of overdose deaths relative to the frequency with which it is prescribed for chronic pain. In addition, methadone is associated with cardiac arrhythmias along with QT prolongation on the electrocardiogram, and it has complicated pharmacokinetics and pharmacodynamics, including a long and variable halflife and peak respiratory depressant effect occurring later and lasting longer than peak analgesic effect. Experts noted that the pharmacodynamics of methadone are subject to more interindividual variability than other opioids. In regard to other ER/ LA opioid formulations, experts noted that the absorption and pharmacodynamics of transdermal fentanyl are complex, with gradually increasing serum concentration during the first part of the 72-hour dosing interval, as well as variable absorption based on factors such as external heat. In addition, the dosing of transdermal fentanyl in mcg/hour, which is not typical for a drug used by outpatients, can be confusing. Experts thought that these complexities might increase the risk for fatal overdose when methadone or transdermal fentanyl is prescribed to a patient who has not used it previously or by clinicians who are not familiar with its effects.

Experts agreed that for patients not already receiving opioids, clinicians should not initiate opioid treatment with ER/LA opioids and should not prescribe ER/LA opioids for intermittent use. ER/LA opioids should be reserved for severe, continuous pain and should be considered only for patients who have received immediate-release opioids daily for at least 1 week. When changing to an ER/LA opioid for a patient previously receiving a different immediate-release opioid, clinicians should consult product labeling and reduce total daily dosage to account for incomplete opioid cross-tolerance. Clinicians should use additional caution with ER/LA opioids and consider a longer dosing interval when prescribing to patients with renal or hepatic dysfunction because decreased clearance of drugs among these patients can lead to accumulation of drugs to toxic levels and persistence in the body for longer durations. Although there might be situations in which clinicians need to prescribe immediate-release and ER/LA opioids together (e.g., transitioning patients from ER/LA opioids to immediate-release opioids by temporarily using lower dosages of both), in general, avoiding the use of immediate-release opioids in combination with ER/LA opioids is preferable, given potentially increased risk and diminishing returns of such an approach for chronic pain.

When an ER/LA opioid is prescribed, using one with predictable pharmacokinetics and pharmacodynamics is preferred to minimize unintentional overdose risk. In particular, unusual characteristics of methadone and of transdermal fentanyl make safe prescribing of these medications for pain especially challenging.

- Methadone should not be the first choice for an ER/LA opioid. Only clinicians who are familiar with methadone's unique risk profile and who are prepared to educate and closely monitor their patients, including risk assessment for QT prolongation and consideration of electrocardiographic monitoring, should consider prescribing methadone for pain. A clinical practice guideline that contains further guidance regarding methadone prescribing for pain has been published previously (191).

- Because dosing effects of transdermal fentanyl are often misunderstood by both clinicians and patients, only clinicians who are familiar with the dosing and absorption properties of transdermal fentanyl and are prepared to educate their patients about its use should consider prescribing it.

5. When opioids are started, clinicians should prescribe the lowest effective dosage. Clinicians should use caution when prescribing opioids at any dosage, should carefully reassess evidence of individual benefits and risks when considering increasing dosage to $\geq 50$ morphine milligram equivalents (MME)/day, and should avoid increasing dosage to $\geq 90 \mathrm{MME} /$ day or carefully justify a decision to titrate dosage to $\geq 90 \mathrm{MME} / \mathrm{day}$ (recommendation category: A, evidence type: 3 ).

Benefits of high-dose opioids for chronic pain are not established. The clinical evidence review found only one study (84) addressing effectiveness of dose titration for outcomes related to pain control, function, and quality of life (KQ3). This randomized trial found no difference in pain or function between a more liberal opioid dose escalation strategy and maintenance of current dosage. (These groups were prescribed average dosages of 52 and $40 \mathrm{MME} / \mathrm{day}$, respectively, at the end of the trial.) At the same time, risks for serious harms 
related to opioid therapy increase at higher opioid dosage. The clinical evidence review found that higher opioid dosages are associated with increased risks for motor vehicle injury, opioid use disorder, and overdose (KQ2). The clinical and contextual evidence reviews found that opioid overdose risk increases in a dose-response manner, that dosages of $50-<100 \mathrm{MME} /$ day have been found to increase risks for opioid overdose by factors of 1.9 to 4.6 compared with dosages of $1-<20 \mathrm{MME} /$ day, and that dosages $\geq 100 \mathrm{MME} /$ day are associated with increased risks of overdose 2.0-8.9 times the risk at 1-<20 MME/day. In a national sample of Veterans Health Administration patients with chronic pain who were prescribed opioids, mean prescribed opioid dosage among patients who died from opioid overdose was $98 \mathrm{MME}$ (median $60 \mathrm{MME}$ ) compared with mean prescribed opioid dosage of $48 \mathrm{MME}$ (median $25 \mathrm{MME}$ ) among patients not experiencing fatal overdose (127).

The contextual evidence review found that although there is not a single dosage threshold below which overdose risk is eliminated, holding dosages $<50 \mathrm{MME} /$ day would likely reduce risk among a large proportion of patients who would experience fatal overdose at higher prescribed dosages. Experts agreed that lower dosages of opioids reduce the risk for overdose, but that a single dosage threshold for safe opioid use could not be identified. Experts noted that daily opioid dosages close to or greater than $100 \mathrm{MME} /$ day are associated with significant risks, that dosages $<50 \mathrm{MME} /$ day are safer than dosages of 50-100 MME/day, and that dosages $<20 \mathrm{MME} /$ day are safer than dosages of 20-50 MME/day. One expert thought that a specific dosage at which the benefit/risk ratio of opioid therapy decreases could not be identified. Most experts agreed that, in general, increasing dosages to 50 or more MME/day increases overdose risk without necessarily adding benefits for pain control or function and that clinicians should carefully reassess evidence of individual benefits and risks when considering increasing opioid dosages to $\geq 50 \mathrm{MME} /$ day. Most experts also agreed that opioid dosages should not be increased to $\geq 90 \mathrm{MME} /$ day without careful justification based on diagnosis and on individualized assessment of benefits and risks.

When opioids are used for chronic pain outside of active cancer, palliative, and end-of-life care, clinicians should start opioids at the lowest possible effective dosage (the lowest starting dosage on product labeling for patients not already taking opioids and according to product labeling guidance regarding tolerance for patients already taking opioids). Clinicians should use additional caution when initiating opioids for patients aged $\geq 65$ years and for patients with renal or hepatic insufficiency because decreased clearance of drugs in these patients can result in accumulation of drugs to toxic levels. Clinicians should use caution when increasing opioid dosages and increase dosage by the smallest practical amount because overdose risk increases with increases in opioid dosage. Although there is limited evidence to recommend specific intervals for dosage titration, a previous guideline recommended waiting at least five half-lives before increasing dosage and waiting at least a week before increasing dosage of methadone to make sure that full effects of the previous dosage are evident (31). Clinicians should re-evaluate patients after increasing dosage for changes in pain, function, and risk for harm (see Recommendation 7). Before increasing total opioid dosage to $\geq 50 \mathrm{MME} /$ day, clinicians should reassess whether opioid treatment is meeting the patient's treatment goals (see Recommendation 2). If a patient's opioid dosage for all sources of opioids combined reaches or exceeds $50 \mathrm{MME} /$ day, clinicians should implement additional precautions, including increased frequency of follow-up (see Recommendation 7) and considering offering naloxone and overdose prevention education to both patients and the patients' household members (see Recommendation 8). Clinicians should avoid increasing opioid dosages to $\geq 90 \mathrm{MME} /$ day or should carefully justify a decision to increase dosage to $\geq 90 \mathrm{MME} /$ day based on individualized assessment of benefits and risks and weighing factors such as diagnosis, incremental benefits for pain and function relative to harms as dosages approach $90 \mathrm{MME} / \mathrm{day}$, other treatments and effectiveness, and recommendations based on consultation with pain specialists. If patients do not experience improvement in pain and function at $\geq 90 \mathrm{MME} /$ day, or if there are escalating dosage requirements, clinicians should discuss other approaches to pain management with the patient, consider working with patients to taper opioids to a lower dosage or to taper and discontinue opioids (see Recommendation 7), and consider consulting a pain specialist. Some states require clinicians to implement clinical protocols at specific dosage levels. For example, before increasing long-term opioid therapy dosage to $>120 \mathrm{MME} /$ day, clinicians in Washington state must obtain consultation from a pain specialist who agrees that this is indicated and appropriate (30). Clinicians should be aware of rules related to MME thresholds and associated clinical protocols established by their states.

Established patients already taking high dosages of opioids, as well as patients transferring from other clinicians, might consider the possibility of opioid dosage reduction to be anxiety-provoking, and tapering opioids can be especially challenging after years on high dosages because of physical and psychological dependence. However, these patients should be offered the opportunity to re-evaluate their continued use of opioids at high dosages in light of recent evidence regarding the association of opioid dosage and overdose risk. Clinicians should explain in a nonjudgmental manner to patients already taking high opioid dosages ( $\geq 90 \mathrm{MME} /$ day) that there is 
now an established body of scientific evidence showing that overdose risk is increased at higher opioid dosages. Clinicians should empathically review benefits and risks of continued high-dosage opioid therapy and should offer to work with the patient to taper opioids to safer dosages. For patients who agree to taper opioids to lower dosages, clinicians should collaborate with the patient on a tapering plan (see Recommendation 7). Experts noted that patients tapering opioids after taking them for years might require very slow opioid tapers as well as pauses in the taper to allow gradual accommodation to lower opioid dosages. Clinicians should remain alert to signs of anxiety, depression, and opioid use disorder (see Recommendations 8 and 12) that might be unmasked by an opioid taper and arrange for management of these co-morbidities. For patients agreeing to taper to lower opioid dosages as well as for those remaining on high opioid dosages, clinicians should establish goals with the patient for continued opioid therapy (see Recommendation 2), maximize pain treatment with nonpharmacologic and nonopioid pharmacologic treatments as appropriate (see Recommendation 1), and consider consulting a pain specialist as needed to assist with pain management.

\section{Long-term opioid use often begins with treatment of} acute pain. When opioids are used for acute pain, clinicians should prescribe the lowest effective dose of immediate-release opioids and should prescribe no greater quantity than needed for the expected duration of pain severe enough to require opioids. Three days or less will often be sufficient; more than seven days will rarely be needed (recommendation category: A, evidence type: 4 ).

The clinical evidence review found that opioid use for acute pain (i.e., pain with abrupt onset and caused by an injury or other process that is not ongoing) is associated with long-term opioid use, and that a greater amount of early opioid exposure is associated with greater risk for long-term use (KQ5). Several guidelines on opioid prescribing for acute pain from emergency departments (192-194) and other settings $(195,196)$ have recommended prescribing $\leq 3$ days of opioids in most cases, whereas others have recommended $\leq 7$ days $(197)$ or $<14$ days (30). Because physical dependence on opioids is an expected physiologic response in patients exposed to opioids for more than a few days (contextual evidence review), limiting days of opioids prescribed also should minimize the need to taper opioids to prevent distressing or unpleasant withdrawal symptoms. Experts noted that more than a few days of exposure to opioids significantly increases hazards, that each day of unnecessary opioid use increases likelihood of physical dependence without adding benefit, and that prescriptions with fewer days' supply will minimize the number of pills available for unintentional or intentional diversion.

Experts agreed that when opioids are needed for acute pain, clinicians should prescribe opioids at the lowest effective dose and for no longer than the expected duration of pain severe enough to require opioids to minimize unintentional initiation of long-term opioid use. The lowest effective dose can be determined using product labeling as a starting point with calibration as needed based on the severity of pain and on other clinical factors such as renal or hepatic insufficiency (see Recommendation 8). Experts thought, based on clinical experience regarding anticipated duration of pain severe enough to require an opioid, that in most cases of acute pain not related to surgery or trauma, a $\leq 3$ days' supply of opioids will be sufficient. For example, in one study of the course of acute low back pain (not associated with malignancies, infections, spondylarthropathies, fractures, or neurological signs) in a primary care setting, there was a large decrease in pain until the fourth day after treatment with paracetamol, with smaller decreases thereafter (198). Some experts thought that because some types of acute pain might require more than 3 days of opioid treatment, it would be appropriate to recommend a range of $\leq 3-5$ days or $\leq 3-7$ days when opioids are needed. Some experts thought that a range including 7 days was too long given the expected course of severe acute pain for most acute pain syndromes seen in primary care.

Acute pain can often be managed without opioids. It is important to evaluate the patient for reversible causes of pain, for underlying etiologies with potentially serious sequelae, and to determine appropriate treatment. When the diagnosis and severity of nontraumatic, nonsurgical acute pain are reasonably assumed to warrant the use of opioids, clinicians should prescribe no greater quantity than needed for the expected duration of pain severe enough to require opioids, often 3 days or less, unless circumstances clearly warrant additional opioid therapy. More than 7 days will rarely be needed. Opioid treatment for post-surgical pain is outside the scope of this guideline but has been addressed elsewhere (30). Clinicians should not prescribe additional opioids to patients "just in case" pain continues longer than expected. Clinicians should re-evaluate the subset of patients who experience severe acute pain that continues longer than the expected duration to confirm or revise the initial diagnosis and to adjust management accordingly. Given longer half-lives and longer duration of effects (e.g., respiratory depression) with ER/LA opioids such as methadone, fentanyl patches, or extended release versions of opioids such as oxycodone, oxymorphone, or morphine, clinicians should not prescribe ER/LA opioids for the treatment of acute pain. 
7. Clinicians should evaluate benefits and harms with patients within 1 to 4 weeks of starting opioid therapy for chronic pain or of dose escalation. Clinicians should evaluate benefits and harms of continued therapy with patients every 3 months or more frequently. If benefits do not outweigh harms of continued opioid therapy, clinicians should optimize other therapies and work with patients to taper opioids to lower dosages or to taper and discontinue opioids (recommendation category: A, evidence type: 4).

Although the clinical evidence review did not find studies evaluating the effectiveness of more frequent monitoring intervals (KQ4), it did find that continuing opioid therapy for 3 months substantially increases risk for opioid use disorder (KQ2); therefore, follow-up earlier than 3 months might be necessary to provide the greatest opportunity to prevent the development of opioid use disorder. In addition, risk for overdose associated with ER/LA opioids might be particularly high during the first 2 weeks of treatment (KQ3). The contextual evidence review found that patients who do not have pain relief with opioids at 1 month are unlikely to experience pain relief with opioids at 6 months. Although evidence is insufficient to determine at what point within the first 3 months of opioid therapy the risks for opioid use disorder increase, reassessment of pain and function within 1 month of initiating opioids provides an opportunity to minimize risks of long-term opioid use by discontinuing opioids among patients not receiving a clear benefit from these medications. Experts noted that risks for opioid overdose are greatest during the first 3-7 days after opioid initiation or increase in dosage, particularly when methadone or transdermal fentanyl are prescribed; that follow-up within 3 days is appropriate when initiating or increasing the dosage of methadone; and that follow-up within 1 week might be appropriate when initiating or increasing the dosage of other ER/LA opioids.

Clinicians should evaluate patients to assess benefits and harms of opioids within 1 to 4 weeks of starting long-term opioid therapy or of dose escalation. Clinicians should consider follow-up intervals within the lower end of this range when ER/LA opioids are started or increased or when total daily opioid dosage is $\geq 50 \mathrm{MME} /$ day. Shorter follow-up intervals (within 3 days) should be strongly considered when starting or increasing the dosage of methadone. At follow up, clinicians should assess benefits in function, pain control, and quality of life using tools such as the three-item "Pain average, interference with Enjoyment of life, and interference with General activity" (PEG) Assessment Scale (186) and/or asking patients about progress toward functional goals that have meaning for them (see Recommendation 2). Clinicians should also ask patients about common adverse effects such as constipation and drowsiness (see Recommendation 3), as well as asking about and assessing for effects that might be early warning signs for more serious problems such as overdose (e.g., sedation or slurred speech) or opioid use disorder (e.g., craving, wanting to take opioids in greater quantities or more frequently than prescribed, or difficulty controlling use). Clinicians should ask patients about their preferences for continuing opioids, given their effects on pain and function relative to any adverse effects experienced.

Because of potential changes in the balance of benefits and risks of opioid therapy over time, clinicians should regularly reassess all patients receiving long-term opioid therapy, including patients who are new to the clinician but on longterm opioid therapy, at least every 3 months. At reassessment, clinicians should determine whether opioids continue to meet treatment goals, including sustained improvement in pain and function, whether the patient has experienced common or serious adverse events or early warning signs of serious adverse events, signs of opioid use disorder (e.g., difficulty controlling use, work or family problems related to opioid use), whether benefits of opioids continue to outweigh risks, and whether opioid dosage can be reduced or opioids can be discontinued. Ideally, these reassessments would take place in person and be conducted by the prescribing clinician. In practice contexts where virtual visits are part of standard care (e.g., in remote areas where distance or other issues make follow-up visits challenging), follow-up assessments that allow the clinician to communicate with and observe the patient through video and audio could be conducted, with in-person visits occurring at least once per year. Clinicians should re-evaluate patients who are exposed to greater risk of opioid use disorder or overdose (e.g., patients with depression or other mental health conditions, a history of substance use disorder, a history of overdose, taking $\geq 50 \mathrm{MME} /$ day, or taking other central nervous system depressants with opioids) more frequently than every 3 months. If clinically meaningful improvements in pain and function are not sustained, if patients are taking high-risk regimens (e.g., dosages $\geq 50 \mathrm{MME} /$ day or opioids combined with benzodiazepines) without evidence of benefit, if patients believe benefits no longer outweigh risks or if they request dosage reduction or discontinuation, or if patients experience overdose or other serious adverse events (e.g., an event leading to hospitalization or disability) or warning signs of serious adverse events, clinicians should work with patients to reduce opioid dosage or to discontinue opioids when possible. Clinicians should maximize pain treatment with nonpharmacologic and nonopioid pharmacologic treatments as appropriate (see Recommendation 1) and consider consulting a pain specialist as needed to assist with pain management. 


\section{Considerations for Tapering Opioids}

Although the clinical evidence review did not find highquality studies comparing the effectiveness of different tapering protocols for use when opioid dosage is reduced or opioids are discontinued (KQ3), tapers reducing weekly dosage by $10 \%-50 \%$ of the original dosage have been recommended by other clinical guidelines (199), and a rapid taper over 2-3 weeks has been recommended in the case of a severe adverse event such as overdose (30). Experts noted that tapers slower than $10 \%$ per week (e.g., $10 \%$ per month) also might be appropriate and better tolerated than more rapid tapers, particularly when patients have been taking opioids for longer durations (e.g., for years). Opioid withdrawal during pregnancy has been associated with spontaneous abortion and premature labor.

When opioids are reduced or discontinued, a taper slow enough to minimize symptoms and signs of opioid withdrawal (e.g., drug craving, anxiety, insomnia, abdominal pain, vomiting, diarrhea, diaphoresis, mydriasis, tremor, tachycardia, or piloerection) should be used. A decrease of $10 \%$ of the original dose per week is a reasonable starting point; experts agreed that tapering plans may be individualized based on patient goals and concerns. Experts noted that at times, tapers might have to be paused and restarted again when the patient is ready and might have to be slowed once patients reach low dosages. Tapers may be considered successful as long as the patient is making progress. Once the smallest available dose is reached, the interval between doses can be extended. Opioids may be stopped when taken less frequently than once a day. More rapid tapers might be needed for patient safety under certain circumstances (e.g., for patients who have experienced overdose on their current dosage). Ultrarapid detoxification under anesthesia is associated with substantial risks, including death, and should not be used (200). Clinicians should access appropriate expertise if considering tapering opioids during pregnancy because of possible risk to the pregnant patient and to the fetus if the patient goes into withdrawal. Patients who are not taking opioids (including patients who are diverting all opioids they obtain) do not require tapers. Clinicians should discuss with patients undergoing tapering the increased risk for overdose on abrupt return to a previously prescribed higher dose. Primary care clinicians should collaborate with mental health providers and with other specialists as needed to optimize nonopioid pain management (see Recommendation 1), as well as psychosocial support for anxiety related to the taper. More detailed guidance on tapering, including management of withdrawal symptoms has been published previously $(30,201)$. If a patient exhibits signs of opioid use disorder, clinicians should offer or arrange for treatment of opioid use disorder (see Recommendation 12) and consider offering naloxone for overdose prevention (see Recommendation 8).

\section{Assessing Risk and Addressing Harms of Opioid Use}

8. Before starting and periodically during continuation of opioid therapy, clinicians should evaluate risk factors for opioid-related harms. Clinicians should incorporate into the management plan strategies to mitigate risk, including considering offering naloxone when factors that increase risk for opioid overdose, such as history of overdose, history of substance use disorder, higher opioid dosages ( $\geq 50 \mathrm{MME} /$ day), or concurrent benzodiazepine use, are present (recommendation category: A, evidence type: 4).

The clinical evidence review found insufficient evidence to determine how harms of opioids differ depending on patient demographics or patient comorbidities (KQ2). However, based on the contextual evidence review and expert opinion, certain risk factors are likely to increase susceptibility to opioidassociated harms and warrant incorporation of additional strategies into the management plan to mitigate risk. Clinicians should assess these risk factors periodically, with frequency varying by risk factor and patient characteristics. For example, factors that vary more frequently over time, such as alcohol use, require more frequent follow up. In addition, clinicians should consider offering naloxone, re-evaluating patients more frequently (see Recommendation 7), and referring to pain and/or behavioral health specialists when factors that increase risk for harm, such as history of overdose, history of substance use disorder, higher dosages of opioids ( $\geq 50 \mathrm{MME} /$ day), and concurrent use of benzodiazepines with opioids, are present.

\section{Patients with Sleep-Disordered Breathing, Including Sleep Apnea}

Risk factors for sleep-disordered breathing include congestive heart failure, and obesity. Experts noted that careful monitoring and cautious dose titration should be used if opioids are prescribed for patients with mild sleep-disordered breathing. Clinicians should avoid prescribing opioids to patients with moderate or severe sleep-disordered breathing whenever possible to minimize risks for opioid overdose (contextual evidence review).

\section{Pregnant Women}

Opioids used in pregnancy might be associated with additional risks to both mother and fetus. Some studies have shown an association of opioid use in pregnancy with stillbirth, poor fetal growth, pre-term delivery, and birth defects (contextual evidence review). Importantly, in some cases, opioid use during pregnancy leads to neonatal opioid withdrawal syndrome. Clinicians and patients together should carefully weigh risks and benefits when making decisions 
about whether to initiate opioid therapy for chronic pain during pregnancy. In addition, before initiating opioid therapy for chronic pain for reproductive-age women, clinicians should discuss family planning and how long-term opioid use might affect any future pregnancy. For pregnant women already receiving opioids, clinicians should access appropriate expertise if considering tapering opioids because of possible risk to the pregnant patient and to the fetus if the patient goes into withdrawal (see Recommendation 7). For pregnant women with opioid use disorder, medication-assisted therapy with buprenorphine or methadone has been associated with improved maternal outcomes and should be offered (202) (see Recommendation 12). Clinicians caring for pregnant women receiving opioids for pain or receiving buprenorphine or methadone for opioid use disorder should arrange for delivery at a facility prepared to monitor, evaluate for, and treat neonatal opioid withdrawal syndrome. In instances when travel to such a facility would present an undue burden on the pregnant woman, it is appropriate to deliver locally, monitor and evaluate the newborn for neonatal opioid withdrawal syndrome, and transfer the newborn for additional treatment if needed. Neonatal toxicity and death have been reported in breastfeeding infants whose mothers are taking codeine (contextual evidence review); previous guidelines have recommended that codeine be avoided whenever possible among mothers who are breast feeding and, if used, should be limited to the lowest possible dose and to a 4-day supply (203).

\section{Patients with Renal or Hepatic Insufficiency}

Clinicians should use additional caution and increased monitoring (see Recommendation 7) to minimize risks of opioids prescribed for patients with renal or hepatic insufficiency, given their decreased ability to process and excrete drugs, susceptibility to accumulation of opioids, and reduced therapeutic window between safe dosages and dosages associated with respiratory depression and overdose (contextual evidence review; see Recommendations 4, 5, and 7).

\section{Patients Aged $\geq 65$ Years}

Inadequate pain treatment among persons aged $\geq 65$ years has been documented (204). Pain management for older patients can be challenging given increased risks of both nonopioid pharmacologic therapies (see Recommendation 1) and opioid therapy in this population. Given reduced renal function and medication clearance even in the absence of renal disease, patients aged $\geq 65$ years might have increased susceptibility to accumulation of opioids and a smaller therapeutic window between safe dosages and dosages associated with respiratory depression and overdose (contextual evidence review). Some older adults suffer from cognitive impairment, which can increase risk for medication errors and make opioid-related confusion more dangerous. In addition, older adults are more likely than younger adults to experience co-morbid medical conditions and more likely to receive multiple medications, some of which might interact with opioids (such as benzodiazepines). Clinicians should use additional caution and increased monitoring (see Recommendations 4, 5, and 7) to minimize risks of opioids prescribed for patients aged $\geq 65$ years. Experts suggested that clinicians educate older adults receiving opioids to avoid risky medication-related behaviors such as obtaining controlled medications from multiple prescribers and saving unused medications. Clinicians should also implement interventions to mitigate common risks of opioid therapy among older adults, such as exercise or bowel regimens to prevent constipation, risk assessment for falls, and patient monitoring for cognitive impairment.

\section{Patients with Mental Health Conditions}

Because psychological distress frequently interferes with improvement of pain and function in patients with chronic pain, using validated instruments such as the Generalized Anxiety Disorder (GAD)-7 and the Patient Health Questionnaire (PHQ)-9 or the PHQ-4 to assess for anxiety, post-traumatic stress disorder, and/or depression (205), might help clinicians improve overall pain treatment outcomes. Experts noted that clinicians should use additional caution and increased monitoring (see Recommendation 7) to lessen the increased risk for opioid use disorder among patients with mental health conditions (including depression, anxiety disorders, and PTSD), as well as increased risk for drug overdose among patients with depression. Previous guidelines have noted that opioid therapy should not be initiated during acute psychiatric instability or uncontrolled suicide risk, and that clinicians should consider behavioral health specialist consultation for any patient with a history of suicide attempt or psychiatric disorder (31). In addition, patients with anxiety disorders and other mental health conditions are more likely to receive benzodiazepines, which can exacerbate opioid-induced respiratory depression and increase risk for overdose (see Recommendation 11). Clinicians should ensure that treatment for depression and other mental health conditions is optimized, consulting with behavioral health specialists when needed. Treatment for depression can improve pain symptoms as well as depression and might decrease overdose risk (contextual evidence review). For treatment of chronic pain in patients with depression, clinicians should strongly consider using tricyclic or SNRI antidepressants for analgesic as well as antidepressant effects if these medications are not otherwise contraindicated (see Recommendation 1). 


\section{Patients with Substance Use Disorder}

Illicit drugs and alcohol are listed as contributory factors on a substantial proportion of death certificates for opioid-related overdose deaths (contextual evidence review). Previous guidelines have recommended screening or risk assessment tools to identify patients at higher risk for misuse or abuse of opioids. However, the clinical evidence review found that currently available riskstratification tools (e.g., Opioid Risk Tool, Screener and Opioid Assessment for Patients with Pain Version 1, SOAPP-R, and Brief Risk Interview) show insufficient accuracy for classification of patients as at low or high risk for abuse or misuse (KQ4). Clinicians should always exercise caution when considering or prescribing opioids for any patient with chronic pain outside of active cancer, palliative, and end-of-life care and should not overestimate the ability of these tools to rule out risks from long-term opioid therapy.

Clinicians should ask patients about their drug and alcohol use. Single screening questions can be used (206). For example, the question "How many times in the past year have you used an illegal drug or used a prescription medication for nonmedical reasons?" (with an answer of one or more considered positive) was found in a primary care setting to be $100 \%$ sensitive and $73.5 \%$ specific for the detection of a drug use disorder compared with a standardized diagnostic interview (207). Validated screening tools such as the Drug Abuse Screening Test (DAST) (208) and the Alcohol Use Disorders Identification Test (AUDIT) (209) can also be used. Clinicians should use PDMP data (see Recommendation 9) and drug testing (see Recommendation 10) as appropriate to assess for concurrent substance use that might place patients at higher risk for opioid use disorder and overdose. Clinicians should also provide specific counseling on increased risks for overdose when opioids are combined with other drugs or alcohol (see Recommendation 3) and ensure that patients receive effective treatment for substance use disorders when needed (see Recommendation 12).

The clinical evidence review found insufficient evidence to determine how harms of opioids differ depending on past or current substance use disorder (KQ2), although a history of substance use disorder was associated with misuse. Similarly, based on contextual evidence, patients with drug or alcohol use disorders are likely to experience greater risks for opioid use disorder and overdose than persons without these conditions. If clinicians consider opioid therapy for chronic pain outside of active cancer, palliative, and end-of-life care for patients with drug or alcohol use disorders, they should discuss increased risks for opioid use disorder and overdose with patients, carefully consider whether benefits of opioids outweigh increased risks, and incorporate strategies to mitigate risk into the management plan, such as considering offering naloxone (see Offering Naloxone to Patients When Factors That Increase Risk for Opioid-Related Harms Are Present) and increasing frequency of monitoring (see Recommendation 7) when opioids are prescribed. Because pain management in patients with substance use disorder can be complex, clinicians should consider consulting substance use disorder specialists and pain specialists regarding pain management for persons with active or recent past history of substance abuse. Experts also noted that clinicians should communicate with patients' substance use disorder treatment providers if opioids are prescribed.

\section{Patients with Prior Nonfatal Overdose}

Although studies were not identified that directly addressed the risk for overdose among patients with prior nonfatal overdose who are prescribed opioids, based on clinical experience, experts thought that prior nonfatal overdose would substantially increase risk for future nonfatal or fatal opioid overdose. If patients experience nonfatal opioid overdose, clinicians should work with them to reduce opioid dosage and to discontinue opioids when possible (see Recommendation 7). If clinicians continue opioid therapy for chronic pain outside of active cancer, palliative, and end-of-life care in patients with prior opioid overdose, they should discuss increased risks for overdose with patients, carefully consider whether benefits of opioids outweigh substantial risks, and incorporate strategies to mitigate risk into the management plan, such as considering offering naloxone (see Offering Naloxone to Patients When Factors That Increase Risk for Opioid-Related Harms Are Present) and increasing frequency of monitoring (see Recommendation 7) when opioids are prescribed.

\section{Offering Naloxone to Patients When Factors That Increase Risk for Opioid-Related Harms Are Present}

Naloxone is an opioid antagonist that can reverse severe respiratory depression; its administration by lay persons, such as friends and family of persons who experience opioid overdose, can save lives. Naloxone precipitates acute withdrawal among patients physically dependent on opioids. Serious adverse effects, such as pulmonary edema, cardiovascular instability, and seizures, have been reported but are rare at doses consistent with labeled use for opioid overdose (210). The contextual evidence review did not find any studies on effectiveness of prescribing naloxone for overdose prevention among patients prescribed opioids for chronic pain. However, there is evidence for effectiveness of naloxone provision in preventing opioid-related overdose death at the community level through community-based distribution (e.g., through overdose education and naloxone distribution programs in community service agencies) to persons at risk for overdose 
(mostly due to illicit opiate use), and it is plausible that effectiveness would be observed when naloxone is provided in the clinical setting as well. Experts agreed that it is preferable not to initiate opioid treatment when factors that increase risk for opioid-related harms are present. Opinions diverged about the likelihood of naloxone being useful to patients and the circumstances under which it should be offered. However, most experts agreed that clinicians should consider offering naloxone when prescribing opioids to patients at increased risk for overdose, including patients with a history of overdose, patients with a history of substance use disorder, patients taking benzodiazepines with opioids (see Recommendation 11), patients at risk for returning to a high dose to which they are no longer tolerant (e.g., patients recently released from prison), and patients taking higher dosages of opioids ( $\geq 50 \mathrm{MME} /$ day). Practices should provide education on overdose prevention and naloxone use to patients receiving naloxone prescriptions and to members of their households. Experts noted that naloxone co-prescribing can be facilitated by clinics or practices with resources to provide naloxone training and by collaborative practice models with pharmacists. Resources for prescribing naloxone in primary care settings can be found through Prescribe to Prevent at http://prescribetoprevent.org.

9. Clinicians should review the patient's history of controlled substance prescriptions using state prescription drug monitoring program (PDMP) data to determine whether the patient is receiving opioid dosages or dangerous combinations that put him or her at high risk for overdose. Clinicians should review PDMP data when starting opioid therapy for chronic pain and periodically during opioid therapy for chronic pain, ranging from every prescription to every 3 months (recommendation category: A, evidence type: 4 ).

PDMPs are state-based databases that collect information on controlled prescription drugs dispensed by pharmacies in most states and, in select states, by dispensing physicians as well. In addition, some clinicians employed by the federal government, including some clinicians in the Indian Health Care Delivery System, are not licensed in the states where they practice, and do not have access to PDMP data. Certain states require clinicians to review PDMP data prior to writing each opioid prescription (see state-level PDMP-related policies on the National Alliance for Model State Drug Laws website at http://www.namsdl.org/prescription-monitoring-programs. cfm). The clinical evidence review did not find studies evaluating the effectiveness of PDMPs on outcomes related to overdose, addiction, abuse, or misuse (KQ4). However, even though evidence is limited on the effectiveness of PDMP implementation at the state level on prescribing and mortality outcomes (28), the contextual evidence review found that most fatal overdoses were associated with patients receiving opioids from multiple prescribers and/or with patients receiving high total daily opioid dosages; information on both of these risk factors for overdose are available to prescribers in the PDMP. PDMP data also can be helpful when patient medication history is not otherwise available (e.g., for patients from other locales) and when patients transition care to a new clinician. The contextual evidence review also found that PDMP information could be used in a way that is harmful to patients. For example, it has been used to dismiss patients from clinician practices (211), which might adversely affect patient safety.

The contextual review found variation in state policies that affect timeliness of PDMP data (and therefore benefits of reviewing PDMP data) as well as time and workload for clinicians in accessing PDMP data. In states that permit delegating access to other members of the health care team, workload for prescribers can be reduced. These differences might result in a different balance of benefits to clinician workload in different states. Experts agreed that PDMPs are useful tools that should be consulted when starting a patient on opioid therapy and periodically during long-term opioid therapy. However, experts disagreed on how frequently clinicians should check the PDMP during long-term opioid therapy, given PDMP access issues and the lag time in reporting in some states. Most experts agreed that PDMP data should be reviewed every 3 months or more frequently during longterm opioid therapy. A minority of experts noted that, given the current burden of accessing PDMP data in some states and the lack of evidence surrounding the most effective interval for PDMP review to improve patient outcomes, annual review of PDMP data during long-term opioid therapy would be reasonable when factors that increase risk for opioid-related harms are not present.

Clinicians should review PDMP data for opioids and other controlled medications patients might have received from additional prescribers to determine whether a patient is receiving high total opioid dosages or dangerous combinations (e.g., opioids combined with benzodiazepines) that put him or her at high risk for overdose. Ideally, PDMP data should be reviewed before every opioid prescription. This is recommended in all states with well-functioning PDMPs and where PDMP access policies make this practicable (e.g., clinician and delegate access permitted), but it is not currently possible in states without functional PDMPs or in those that do not permit certain prescribers to access them. As vendors and practices facilitate integration of PDMP information into regular clinical workflow (e.g., data made available in electronic health records), clinicians' ease of access in reviewing PDMP data is expected to improve. 
In addition, improved timeliness of PDMP data will improve their value in identifying patient risks.

If patients are found to have high opioid dosages, dangerous combinations of medications, or multiple controlled substance prescriptions written by different clinicians, several actions can be taken to augment clinicians' abilities to improve patient safety:

- Clinicians should discuss information from the PDMP with their patient and confirm that the patient is aware of the additional prescriptions. Occasionally, PDMP information can be incorrect (e.g., if the wrong name or birthdate has been entered, the patient uses a nickname or maiden name, or another person has used the patient's identity to obtain prescriptions).

- Clinicians should discuss safety concerns, including increased risk for respiratory depression and overdose, with patients found to be receiving opioids from more than one prescriber or receiving medications that increase risk when combined with opioids (e.g., benzodiazepines) and consider offering naloxone (see Recommendation 8).

- Clinicians should avoid prescribing opioids and benzodiazepines concurrently whenever possible. Clinicians should communicate with others managing the patient to discuss the patient's needs, prioritize patient goals, weigh risks of concurrent benzodiazepine and opioid exposure, and coordinate care (see Recommendation 11).

- Clinicians should calculate the total MME/day for concurrent opioid prescriptions to help assess the patient's overdose risk (see Recommendation 5). If patients are found to be receiving high total daily dosages of opioids, clinicians should discuss their safety concerns with the patient, consider tapering to a safer dosage (see Recommendations 5 and 7), and consider offering naloxone (see Recommendation 8).

- Clinicians should discuss safety concerns with other clinicians who are prescribing controlled substances for their patient. Ideally clinicians should first discuss concerns with their patient and inform him or her that they plan to coordinate care with the patient's other prescribers to improve the patient's safety.

- Clinicians should consider the possibility of a substance use disorder and discuss concerns with their patient (see Recommendation 12).

- If clinicians suspect their patient might be sharing or selling opioids and not taking them, clinicians should consider urine drug testing to assist in determining whether opioids can be discontinued without causing withdrawal (see Recommendations 7 and 10). A negative drug test for prescribed opioids might indicate the patient is not taking prescribed opioids, although clinicians should consider other possible reasons for this test result (see Recommendation 10).

Experts agreed that clinicians should not dismiss patients from their practice on the basis of PDMP information. Doing so can adversely affect patient safety, could represent patient abandonment, and could result in missed opportunities to provide potentially lifesaving information (e.g., about risks of opioids and overdose prevention) and interventions (e.g., safer prescriptions, nonopioid pain treatment [see Recommendation 1], naloxone [see Recommendation 8], and effective treatment for substance use disorder [see Recommendation 12]).

\section{When prescribing opioids for chronic pain, clinicians should use urine drug testing before starting opioid therapy and consider urine drug testing at least annually to assess for prescribed medications as well as other controlled prescription drugs and illicit drugs (recommendation category: B, evidence type: 4).}

Concurrent use of opioid pain medications with other opioid pain medications, benzodiazepines, or heroin can increase patients' risk for overdose. Urine drug tests can provide information about drug use that is not reported by the patient. In addition, urine drug tests can assist clinicians in identifying when patients are not taking opioids prescribed for them, which might in some cases indicate diversion or other clinically important issues such as difficulties with adverse effects. Urine drug tests do not provide accurate information about how much or what dose of opioids or other drugs a patient took. The clinical evidence review did not find studies evaluating the effectiveness of urine drug screening for risk mitigation during opioid prescribing for pain (KQ4). The contextual evidence review found that urine drug testing can provide useful information about patients assumed not to be using unreported drugs. Urine drug testing results can be subject to misinterpretation and might sometimes be associated with practices that might harm patients (e.g., stigmatization, inappropriate termination from care). Routine use of urine drug tests with standardized policies at the practice or clinic level might destigmatize their use. Although random drug testing also might destigmatize urine drug testing, experts thought that truly random testing was not feasible in clinical practice. Some clinics obtain a urine specimen at every visit, but only send it for testing on a random schedule. Experts noted that in addition to direct costs of urine drug testing, which often are not covered fully by insurance and can be a burden for patients, clinician time is needed to interpret, confirm, and communicate results.

Experts agreed that prior to starting opioids for chronic pain and periodically during opioid therapy, clinicians should 
use urine drug testing to assess for prescribed opioids as well as other controlled substances and illicit drugs that increase risk for overdose when combined with opioids, including nonprescribed opioids, benzodiazepines, and heroin. There was some difference of opinion among experts as to whether this recommendation should apply to all patients, or whether this recommendation should entail individual decision making with different choices for different patients based on values, preferences, and clinical situations. While experts agreed that clinicians should use urine drug testing before initiating opioid therapy for chronic pain, they disagreed on how frequently urine drug testing should be conducted during long-term opioid therapy. Most experts agreed that urine drug testing at least annually for all patients was reasonable. Some experts noted that this interval might be too long in some cases and too short in others, and that the follow-up interval should be left to the discretion of the clinician. Previous guidelines have recommended more frequent urine drug testing in patients thought to be at higher risk for substance use disorder (30). However, experts thought that predicting risk prior to urine drug testing is challenging and that currently available tools do not allow clinicians to reliably identify patients who are at low risk for substance use disorder.

In most situations, initial urine drug testing can be performed with a relatively inexpensive immunoassay panel for commonly prescribed opioids and illicit drugs. Patients prescribed less commonly used opioids might require specific testing for those agents. The use of confirmatory testing adds substantial costs and should be based on the need to detect specific opioids that cannot be identified on standard immunoassays or on the presence of unexpected urine drug test results. Clinicians should be familiar with the drugs included in urine drug testing panels used in their practice and should understand how to interpret results for these drugs. For example, a positive "opiates" immunoassay detects morphine, which might reflect patient use of morphine, codeine, or heroin, but this immunoassay does not detect synthetic opioids (e.g., fentanyl or methadone) and might not detect semisynthetic opioids (e.g., oxycodone). However, many laboratories use an oxycodone immunoassay that detects oxycodone and oxymorphone. In some cases, positive results for specific opioids might reflect metabolites from opioids the patient is taking and might not mean the patient is taking the specific opioid for which the test was positive. For example, hydromorphone is a metabolite of hydrocodone, and oxymorphone is a metabolite of oxycodone. Detailed guidance on interpretation of urine drug test results, including which tests to order and expected results, drug detection time in urine, drug metabolism, and other considerations has been published previously (30). Clinicians should not test for substances for which results would not affect patient management or for which implications for patient management are unclear. For example, experts noted that there might be uncertainty about the clinical implications of a positive urine drug test for tetrahyrdocannabinol (THC). In addition, restricting confirmatory testing to situations and substances for which results can reasonably be expected to affect patient management can reduce costs of urine drug testing, given the substantial costs associated with confirmatory testing methods. Before ordering urine drug testing, clinicians should have a plan for responding to unexpected results. Clinicians should explain to patients that urine drug testing is intended to improve their safety and should also explain expected results (e.g., presence of prescribed medication and absence of drugs, including illicit drugs, not reported by the patient). Clinicians should ask patients about use of prescribed and other drugs and ask whether there might be unexpected results. This will provide an opportunity for patients to provide information about changes in their use of prescribed opioids or other drugs. Clinicians should discuss unexpected results with the local laboratory or toxicologist and with the patient. Discussion with patients prior to specific confirmatory testing can sometimes yield a candid explanation of why a particular substance is present or absent and obviate the need for expensive confirmatory testing on that visit. For example, a patient might explain that the test is negative for prescribed opioids because she felt opioids were no longer helping and discontinued them. If unexpected results are not explained, a confirmatory test using a method selective enough to differentiate specific opioids and metabolites (e.g., gas or liquid chromatography/mass spectrometry) might be warranted to clarify the situation.

Clinicians should use unexpected results to improve patient safety (e.g., change in pain management strategy [see Recommendation 1], tapering or discontinuation of opioids [see Recommendation 7], more frequent re-evaluation [see Recommendation 7], offering naloxone [see Recommendation 8], or referral for treatment for substance use disorder [see Recommendation 12], all as appropriate). If tests for prescribed opioids are repeatedly negative, confirming that the patient is not taking the prescribed opioid, clinicians can discontinue the prescription without a taper. Clinicians should not dismiss patients from care based on a urine drug test result because this could constitute patient abandonment and could have adverse consequences for patient safety, potentially including the patient obtaining opioids from alternative sources and the clinician missing opportunities to facilitate treatment for substance use disorder.

\section{Clinicians should avoid prescribing opioid pain medication and benzodiazepines concurrently}


whenever possible (recommendation category: A, evidence type: 3 ).

Benzodiazepines and opioids both cause central nervous system depression and can decrease respiratory drive. Concurrent use is likely to put patients at greater risk for potentially fatal overdose. The clinical evidence review did not address risks of benzodiazepine co-prescription among patients prescribed opioids. However, the contextual evidence review found evidence in epidemiologic series of concurrent benzodiazepine use in large proportions of opioid-related overdose deaths, and a case-cohort study found concurrent benzodiazepine prescription with opioid prescription to be associated with a near quadrupling of risk for overdose death compared with opioid prescription alone (212). Experts agreed that although there are circumstances when it might be appropriate to prescribe opioids to a patient receiving benzodiazepines (e.g., severe acute pain in a patient taking longterm, stable low-dose benzodiazepine therapy), clinicians should avoid prescribing opioids and benzodiazepines concurrently whenever possible. In addition, given that other central nervous system depressants (e.g., muscle relaxants, hypnotics) can potentiate central nervous system depression associated with opioids, clinicians should consider whether benefits outweigh risks of concurrent use of these drugs. Clinicians should check the PDMP for concurrent controlled medications prescribed by other clinicians (see Recommendation 9) and should consider involving pharmacists and pain specialists as part of the management team when opioids are co-prescribed with other central nervous system depressants. Because of greater risks of benzodiazepine withdrawal relative to opioid withdrawal, and because tapering opioids can be associated with anxiety, when patients receiving both benzodiazepines and opioids require tapering to reduce risk for fatal respiratory depression, it might be safer and more practical to taper opioids first (see Recommendation 7). Clinicians should taper benzodiazepines gradually if discontinued because abrupt withdrawal can be associated with rebound anxiety, hallucinations, seizures, delirium tremens, and, in rare cases, death (contextual evidence review). A commonly used tapering schedule that has been used safely and with moderate success is a reduction of the benzodiazepine dose by $25 \%$ every 1-2 weeks $(213,214)$. CBT increases tapering success rates and might be particularly helpful for patients struggling with a benzodiazepine taper (213). If benzodiazepines prescribed for anxiety are tapered or discontinued, or if patients receiving opioids require treatment for anxiety, evidence-based psychotherapies (e.g., CBT) and/or specific anti-depressants or other nonbenzodiazepine medications approved for anxiety should be offered. Experts emphasized that clinicians should communicate with mental health professionals managing the patient to discuss the patient's needs, prioritize patient goals, weigh risks of concurrent benzodiazepine and opioid exposure, and coordinate care.

12. Clinicians should offer or arrange evidence-based treatment (usually medication-assisted treatment with buprenorphine or methadone in combination with behavioral therapies) for patients with opioid use disorder (recommendation category: A, evidence type: 2 ).

Opioid use disorder (previously classified as opioid abuse or opioid dependence) is defined in the Diagnostic and Statistical Manual of Mental Disorders, 5th edition (DSM-5) as a problematic pattern of opioid use leading to clinically significant impairment or distress, manifested by at least two defined criteria occurring within a year (http://pcssmat. org/wp-content/uploads/2014/02/5B-DSM-5-Opioid-UseDisorder-Diagnostic-Criteria.pdf) (20).

The clinical evidence review found prevalence of opioid dependence (using DSM-IV diagnosis criteria) in primary care settings among patients with chronic pain on opioid therapy to be $3 \%-26 \%$ (KQ2). As found in the contextual evidence review and supported by moderate quality evidence, opioid agonist or partial agonist treatment with methadone maintenance therapy or buprenorphine has been shown to be more effective in preventing relapse among patients with opioid use disorder (151-153). Some studies suggest that using behavioral therapies in combination with these treatments can reduce opioid misuse and increase retention during maintenance therapy and improve compliance after detoxification $(154,155)$; behavioral therapies are also recommended by clinical practice guidelines (215). The cited studies primarily evaluated patients with a history of illicit opioid use, rather than prescription opioid use for chronic pain. Recent studies among patients with prescription opioid dependence (based on DSM-IV criteria) have found maintenance therapy with buprenorphine and buprenorphinenaloxone effective in preventing relapse $(216,217)$. Treatment need in a community is often not met by capacity to provide buprenorphine or methadone maintenance therapy (218), and patient cost can be a barrier to buprenorphine treatment because insurance coverage of buprenorphine for opioid use disorder is often limited (219). Oral or long-acting injectable formulations of naltrexone can also be used as medicationassisted treatment for opioid use disorder in nonpregnant adults, particularly for highly motivated persons $(220,221)$. Experts agreed that clinicians prescribing opioids should identify treatment resources for opioid use disorder in the community and should work together to ensure sufficient treatment capacity for opioid use disorder at the practice level. 
If clinicians suspect opioid use disorder based on patient concerns or behaviors or on findings in prescription drug monitoring program data (see Recommendation 9) or from urine drug testing (see Recommendation 10), they should discuss their concern with their patient and provide an opportunity for the patient to disclose related concerns or problems. Clinicians should assess for the presence of opioid use disorder using DSM-5 criteria (20). Alternatively, clinicians can arrange for a substance use disorder treatment specialist to assess for the presence of opioid use disorder. For patients meeting criteria for opioid use disorder, clinicians should offer or arrange for patients to receive evidence-based treatment, usually medication-assisted treatment with buprenorphine or methadone maintenance therapy in combination with behavioral therapies. Oral or long-acting injectable naltrexone, a long-acting opioid antagonist, can also be used in nonpregnant adults. Naltrexone blocks the effects of opioids if they are used but requires adherence to daily oral therapy or monthly injections. For pregnant women with opioid use disorder, medication-assisted therapy with buprenorphine (without naloxone) or methadone has been associated with improved maternal outcomes and should be offered (see Recommendation 8). Clinicians should also consider offering naloxone for overdose prevention to patients with opioid use disorder (see Recommendation 8). For patients with problematic opioid use that does not meet criteria for opioid use disorder, experts noted that clinicians can offer to taper and discontinue opioids (see Recommendation 7). For patients who choose to but are unable to taper, clinicians may reassess for opioid use disorder and offer opioid agonist therapy if criteria are met.

Physicians not already certified to provide buprenorphine in an office-based setting can undergo training to receive a waiver from the Substance Abuse and Mental Health Services Administration (SAMHSA) that allows them to prescribe buprenorphine to treat patients with opioid use disorder. Physicians prescribing opioids in communities without sufficient treatment capacity for opioid use disorder should strongly consider obtaining this waiver. Information about qualifications and the process to obtain a waiver are available from SAMHSA (222). Clinicians do not need a waiver to offer naltrexone for opioid use disorder as part of their practice.

Additional guidance has been published previously (215) on induction, use, and monitoring of buprenorphine treatment (see Part 5) and naltrexone treatment (see Part 6) for opioid use disorder and on goals, components of, and types of effective psychosocial treatment that are recommended in conjunction with pharmacological treatment of opioid use disorder (see Part 7). Clinicians unable to provide treatment themselves should arrange for patients with opioid use disorder to receive care from a substance use disorder treatment specialist, such as an office-based buprenorphine or naltrexone treatment provider, or from an opioid treatment program certified by SAMHSA to provide supervised medication-assisted treatment for patients with opioid use disorder. Clinicians should assist patients in finding qualified treatment providers and should arrange for patients to follow up with these providers, as well as arranging for ongoing coordination of care. Clinicians should not dismiss patients from their practice because of a substance use disorder because this can adversely affect patient safety and could represent patient abandonment. Identification of substance use disorder represents an opportunity for a clinician to initiate potentially life-saving interventions, and it is important for the clinician to collaborate with the patient regarding their safety to increase the likelihood of successful treatment. In addition, although identification of an opioid use disorder can alter the expected benefits and risks of opioid therapy for pain, patients with co-occurring pain and substance use disorder require ongoing pain management that maximizes benefits relative to risks. Clinicians should continue to use nonpharmacologic and nonopioid pharmacologic pain treatments as appropriate (see Recommendation 1) and consider consulting a pain specialist as needed to provide optimal pain management.

Resources to help with arranging for treatment include SAMHSA's buprenorphine physician locator (http:// buprenorphine.samhsa.gov/bwns_locator); SAMHSA's Opioid Treatment Program Directory (http://dpt2.samhsa. gov/treatment/directory.aspx); SAMHSA's Provider Clinical Support System for Opioid Therapies (http://pcss-o.org), which offers extensive experience in the treatment of substance use disorders and specifically of opioid use disorder, as well as expertise on the interface of pain and opioid misuse; and SAMHSA's Provider's Clinical Support System for MedicationAssisted Treatment (http://pcssmat.org), which offers expert physician mentors to answer questions about assessment for and treatment of substance use disorders.

\section{Conclusions and Future Directions}

Clinical guidelines represent one strategy for improving prescribing practices and health outcomes. Efforts are required to disseminate the guideline and achieve widespread adoption and implementation of the recommendations in clinical settings. CDC will translate this guideline into user-friendly materials for distribution and use by health systems, medical professional societies, insurers, public health departments, health information technology developers, and clinicians and engage in dissemination efforts. CDC has provided a 
checklist for prescribing opioids for chronic pain (http:// stacks.cdc.gov/view/cdc/38025), additional resources such as fact sheets (http://www.cdc.gov/drugoverdose/prescribing/ resources.html), and will provide a mobile application to guide clinicians in implementing the recommendations. CDC will also work with partners to support clinician education on pain management options, opioid therapy, and risk mitigation strategies (e.g., urine drug testing). Activities such as development of clinical decision support in electronic health records to assist clinicians' treatment decisions at the point of care; identification of mechanisms that insurers and pharmacy benefit plan managers can use to promote safer prescribing within plans; and development of clinical quality improvement measures and initiatives to improve prescribing and patient care within health systems have promise for increasing guideline adoption and improving practice. In addition, policy initiatives that address barriers to implementation of the guidelines, such as increasing accessibility of PDMP data within and across states, e-prescribing, and availability of clinicians who can offer medication-assisted treatment for opioid use disorder, are strategies to consider to enhance implementation of the recommended practices. CDC will work with federal partners and payers to evaluate strategies such as payment reform and health care delivery models that could improve patient health and safety. For example, strategies might include strengthened coverage for nonpharmacologic treatments, appropriate urine drug testing, and medication-assisted treatment; reimbursable time for patient counseling; and payment models that improve access to interdisciplinary, coordinated care.

As highlighted in the forthcoming report on the National Pain Strategy, an overarching federal effort that outlines a comprehensive population-level health strategy for addressing pain as a public health problem, clinical guidelines complement other strategies aimed at preventing illnesses and injuries that lead to pain. A draft of the National Pain Strategy has been published previously (180). These strategies include strengthening the evidence base for pain prevention and treatment strategies, reducing disparities in pain treatment, improving service delivery and reimbursement, supporting professional education and training, and providing public education. It is important that overall improvements be made in developing the workforce to address pain management in general, in addition to opioid prescribing specifically. This guideline also complements other federal efforts focused on addressing the opioid overdose epidemic including prescriber training and education, improving access to treatment for opioid use disorder, safe storage and disposal programs, utilization management mechanisms, naloxone distribution programs, law enforcement and supply reduction efforts, prescription drug monitoring program improvements, and support for community coalitions and state prevention programs.

This guideline provides recommendations that are based on the best available evidence that was interpreted and informed by expert opinion. The clinical scientific evidence informing the recommendations is low in quality. To inform future guideline development, more research is necessary to fill in critical evidence gaps. The evidence reviews forming the basis of this guideline clearly illustrate that there is much yet to be learned about the effectiveness, safety, and economic efficiency of long-term opioid therapy. As highlighted by an expert panel in a recent workshop sponsored by the National Institutes of Health on the role of opioid pain medications in the treatment of chronic pain, "evidence is insufficient for every clinical decision that a provider needs to make about the use of opioids for chronic pain" (223). The National Institutes of Health panel recommended that research is needed to improve our understanding of which types of pain, specific diseases, and patients are most likely to be associated with benefit and harm from opioid pain medications; evaluate multidisciplinary pain interventions; estimate cost-benefit; develop and validate tools for identification of patient risk and outcomes; assess the effectiveness and harms of opioid pain medications with alternative study designs; and investigate risk identification and mitigation strategies and their effects on patient and public health outcomes. It is also important to obtain data to inform the cost feasibility and cost-effectiveness of recommended actions, such as use of nonpharmacologic therapy and urine drug testing. Research that contributes to safer and more effective pain treatment can be implemented across public health entities and federal agencies (4). Additional research can inform the development of future guidelines for special populations that could not be adequately addressed in this guideline, such as children and adolescents, where evidence and guidance is needed but currently lacking. CDC is committed to working with partners to identify the highest priority research areas to build the evidence base. Yet, given that chronic pain is recognized as a significant public health problem, the risks associated with long-term opioid therapy, the availability of effective nonpharmacological and nonopioid pharmacologic treatment options for pain, and the potential for improvement in the quality of health care with the implementation of recommended practices, a guideline for prescribing is warranted with the evidence that is currently available. The balance between the benefits and the risks of long-term opioid therapy for chronic pain based on both clinical and contextual evidence is strong enough to support the issuance of category A recommendations in most cases. 
CDC will revisit this guideline as new evidence becomes available to determine when evidence gaps have been sufficiently closed to warrant an update of the guideline. Until this research is conducted, clinical practice guidelines will have to be based on the best available evidence and expert opinion. This guideline is intended to improve communication between clinicians and patients about the risks and benefits of opioid therapy for chronic pain, improve the safety and effectiveness of pain treatment, and reduce the risks associated with longterm opioid therapy, including opioid use disorder, overdose, and death. CDC is committed to evaluating the guideline to identify the impact of the recommendations on clinician and patient outcomes, both intended and unintended, and revising the recommendations in future updates when warranted.

\section{Acknowledgments}

Members of the Core Expert Group; the Core Expert Group facilitator: Don Teater, MD; members of the Stakeholder Review Group; peer reviewers; the Opioid Guideline Workgroup, consultants, and the NCIPC Board of Scientific Counselors; federal partners: Richard Kronick, PhD, Deborah G. Perfetto, PharmD, Agency for Healthcare Research and Quality; Jeffrey A. Kelman, MD, Diane L. McNally, Centers for Medicare \& Medicaid Services; Jonathan Woodson, MD, Dave Smith, MD, Jack Smith, MD, Christopher Spevak, MD, Department of Defense; Stephen M. Ostroff, MD, Christopher M. Jones, PharmD, Food and Drug Administration; Jim Macrae, MA, MPP, Alexander F. Ross, ScD, Health Resources and Services Administration; Nora Volkow, MD, David Thomas, PhD, National Institute of Drug Abuse; John Howard, MD, Douglas Trout, MD, National Institute for Occupational Safety and Health; Karen B. DeSalvo, MD, Jennifer Frazier, MPH, Office of the National Coordinator, Michael Botticelli, MEd, Cecelia McNamara Spitznas, PhD, Office of National Drug Control Policy; Kana Enomoto, MA, Jinhee Lee, PharmD, Substance Abuse and Mental Health Services Administration; Robert McDonald, MBA, Jack M. Rosenberg, MD, Veterans Administration; members of the public who provided comment during the webinar; Douglas McDonald, PhD, Brandy Wyant, MPH, Kenneth Carlson, Amy Berninger, MPH, Abt Associates; Thomas Frieden, MD, Anne Schuchat, MD, Ileana Arias, PhD, CDC Office of the Director, Debra Houry, MD, National Center for Injury Prevention and Control, Amy Peeples, MPA, National Center for Injury Prevention and Control, Arlene Greenspan, DrPH, National Center for Injury Prevention and Control, Grant Baldwin, PhD, Division of Unintentional Injury Prevention, National Center for Injury Prevention and Control, Rita Noonan, PhD, Division of Unintentional Injury Prevention, National Center for Injury Prevention and Control, Julie Gilchrist, MD, Division of Unintentional Injury Prevention, National Center for Injury Prevention and Control, Terry Davis, EdD, Division of Unintentional Injury Prevention, National
Center for Injury Prevention and Control, Wes Sargent, EdD, Division of Unintentional Injury Prevention, National Center for Injury Prevention and Control, Brian Manns, PharmD, Division of Unintentional Injury Prevention, National Center for Injury Prevention and Control, Lisa Garbarino, Division of Unintentional Injury Prevention, National Center for Injury Prevention and Control, Donovan Newton, MPA, Division of Analysis, Research and Practice Integration, National Center for Injury Prevention and Control, Joann Kang, JD, Division of Unintentional Injury Prevention, National Center for Injury Prevention and Control, Noah Aleshire, JD, Division of Unintentional Injury Prevention, National Center for Injury Prevention and Control, Jennifer VanderVeur, JD, Division of Unintentional Injury Prevention, National Center for Injury Prevention and Control, LeShaundra Scott, MPH, Division of Unintentional Injury Prevention, National Center for Injury Prevention and Control, Sarah Lewis, MPH, Division of Unintentional Injury Prevention, National Center for Injury Prevention and Control, Helen Kingery, MPH, Division of Unintentional Injury Prevention, National Center for Injury Prevention and Control, Kristen Sanderson, MPH, Division of Unintentional Injury Prevention, National Center for Injury Prevention and Control, Kate Fox, MPP, National Center for Injury Prevention and Control, Leslie Dorigo, MA, National Center for Injury Prevention and Control, Erin Connelly, MPA, National Center for Injury Prevention and Control, Sara Patterson, MA, National Center for Injury Prevention and Control, Mark Biagioni, MPA, National Center for Injury Prevention and Control, and Leonard J. Paulozzi, MD, Division of Unintentional Injury Prevention, National Center for Injury Prevention and Control, CDC.

\section{References}

1. Daubresse M, Chang HY, Yu Y, et al. Ambulatory diagnosis and treatment of nonmalignant pain in the United States, 2000-2010. Med Care 2013;51:870-8. http://dx.doi.org/10.1097/MLR.0b013e3182a95d86

2. Paulozzi LJ, Mack KA, Hockenberry JM. Vital signs: variation among states in prescribing of opioid pain relievers and benzodiazepines-United States, 2012. MMWR Morb Mortal Wkly Rep 2014;63:563-8.

3. Levy B, Paulozzi L, Mack KA, Jones CM. Trends in opioid analgesicprescribing rates by specialty, U.S., 2007-2012. Am J Prev Med 2015;49:409-13. http://dx.doi.org/10.1016/j.amepre.2015.02.020

4. Institute of Medicine. Relieving pain in America: a blueprint for transforming prevention, care, education, and research. Washington, DC: The National Academies Press; 2011.

5. International Association for the Study of Pain. Classification of chronic pain. Descriptions of chronic pain syndromes and definitions of pain terms. Prepared by the International Association for the Study of Pain, Subcommittee on Taxonomy. Pain Suppl 1986;3:S1-226.

6. Hardt J, Jacobsen C, Goldberg J, Nickel R, Buchwald D. Prevalence of chronic pain in a representative sample in the United States. Pain Med 2008;9:803-12. http://dx.doi.org/10.1111/j.1526-4637.2008.00425.x

7. Tsang A, Von Korff M, Lee $S$, et al. Common chronic pain conditions in developed and developing countries: gender and age differences and comorbidity with depression-anxiety disorders. J Pain 2008;9:883-91. Corrected in: Demytteneare K. J Pain 2009;10:553. http://dx.doi. org/10.1016/j.jpain.2008.05.005 
8. Nahin RL. Estimates of pain prevalence and severity in adults: United States, 2012. J Pain 2015;16:769-80. http://dx.doi.org/10.1016/j. jpain.2015.05.002

9. Furlan A, Chaparro LE, Irvin E, Mailis-Gagnon A. A comparison between enriched and nonenriched enrollment randomized withdrawal trials of opioids for chronic noncancer pain. Pain Res Manag 2011;16:337-51.

10. American Pain Society, American Academy of Pain Medicine Opioids Guidelines Panel. Guideline for the use of chronic opioid therapy in chronic noncancer pain: evidence review. Chicago, IL: American Pain Society; 2009. http://americanpainsociety.org/uploads/education/ guidelines/chronic-opioid-therapy-cncp.pdf

11. Anastassopoulos KP, Chow W, Tapia CI, Baik R, Moskowitz B, Kim MS. Reported side effects, bother, satisfaction, and adherence in patients taking hydrocodone for non-cancer pain. J Opioid Manag 2013;9:97109. http://dx.doi.org/10.5055/jom.2012.0151

12. Gregorian RS Jr, Gasik A, Kwong WJ, Voeller S, Kavanagh S. Importance of side effects in opioid treatment: a trade-off analysis with patients and physicians. J Pain 2010;11:1095-108. http://dx.doi. org/10.1016/j.jpain.2010.02.007

13. Thielke SM, Turner JA, Shortreed SM, et al. Do patient-perceived pros and cons of opioids predict sustained higher-dose use? Clin J Pain 2014;30:93-101.

14. Chou R, Deyo R, Devine B, et al. The effectiveness and risks of longterm opioid treatment of chronic pain. Evidence Report/Technology Assessment No. 218. AHRQ Publication No. 14-E005-EF. Rockville, MD: Agency for Healthcare Research and Quality; 2014. http://www. effectivehealthcare.ahrq.gov/ehc/products/557/1971/chronic-painopioid-treatment-report-141007.pdf

15. Boudreau D, Von Korff M, Rutter CM, et al. Trends in long-term opioid therapy for chronic non-cancer pain. Pharmacoepidemiol Drug Saf 2009;18:1166-75. http://dx.doi.org/10.1002/pds.1833

16. CDC. Multiple cause of death data on CDC WONDER. Atlanta, GA: US Department of Health and Human Services, CDC; 2016. http:// wonder.cdc.gov/mcd.html

17. CDC, National Center for Health Statistics. Health, United States, 2014: with special feature on adults aged 55-64. Hyattsville, MD: US Department of Health and Human Services, CDC, National Center for Health Statistics; 2015.

18. CDC. Vital signs: overdoses of prescription opioid pain relieversUnited States, 1999-2008. MMWR Morb Mortal Wkly Rep 2011;60:1487-92.

19. Substance Abuse and Mental Health Services Administration. The DAWN report: highlights of the 2011 Drug Abuse Warning Network (DAWN) findings on drug-related emergency department visits. Rockville, MD: US Department of Health and Human Services, Substance Abuse and Mental Health Services Administration, Center for Behavioral Health Statistics and Quality; 2013.

20. American Psychiatric Association. Diagnostic and statistical manual of mental disorders. 5th ed. Arlington, VA: American Psychiatric Publishing; 2013.

21. Substance Abuse and Mental Health Services Administration. Results from the 2013 National Survey on Drug Use and Health: summary of national findings. Rockville, MD: US Department of Health and Human Services, Substance Abuse and Mental Health Services Administration; 2014.
22. Edlund MJ, Martin BC, Russo JE, DeVries A, Braden JB, Sullivan MD. The role of opioid prescription in incident opioid abuse and dependence among individuals with chronic noncancer pain: the role of opioid prescription. Clin J Pain 2014;30:557-64.

23. Zedler B, Xie L, Wang L, et al. Risk factors for serious prescription opioid-related toxicity or overdose among Veterans Health Administration patients. Pain Med 2014;15:1911-29. http://dx.doi. org/10.1111/pme. 12480

24. Bohnert AS, Valenstein M, Bair MJ, et al. Association between opioid prescribing patterns and opioid overdose-related deaths. JAMA 2011;305:1315-21. http://dx.doi.org/10.1001/jama.2011.370

25. Kaplovitch E, Gomes T, Camacho X, Dhalla IA, Mamdani MM, Juurlink DN. Sex differences in dose escalation and overdose death during chronic opioid therapy: a population-based cohort study. PLoS One 2015;10:e0134550. http://dx.doi.org/10.1371/journal. pone. 0134550

26. Jamison RN, Sheehan KA, Scanlan E, Matthews M, Ross EL. Beliefs and attitudes about opioid prescribing and chronic pain management: survey of primary care providers. J Opioid Manag 2014;10:375-82. http://dx.doi.org/10.5055/jom.2014.0234

27. Wilson HD, Dansie EJ, Kim MS, Moskovitz BL, Chow W, Turk DC. Clinicians' attitudes and beliefs about opioids survey (CAOS): instrument development and results of a national physician survey. J Pain 2013;14:613-27. http://dx.doi.org/10.1016/j.jpain.2013.01.769

28. Haegerich TM, Paulozzi LJ, Manns BJ, Jones CM. What we know, and don't know, about the impact of state policy and systems-level interventions on prescription drug overdose. Drug Alcohol Depend 2014;145:34-47. http://dx.doi.org/10.1016/j.drugalcdep.2014.10.001

29. Chou R, Fanciullo GJ, Fine PG, et al.; American Pain Society-American Academy of Pain Medicine Opioids Guidelines Panel. Clinical guidelines for the use of chronic opioid therapy in chronic noncancer pain. J Pain 2009;10:113-30.e22. http://dx.doi.org/10.1016/j. jpain.2008.10.008

30. Washington State Agency Medical Directors' Group. AMDG 2015 interagency guideline on prescribing opioids for pain. Olympia, WA: Washington State Agency Medical Directors' Group; 2015. http:// www.agencymeddirectors.wa.gov/guidelines.asp

31. US Department of Veterans Affairs. VA/DoD clinical practice guidelines: management of opioid therapy (OT) for chronic pain. Washington, DC: US Department of Veterans Affairs; 2010. http:// www.healthquality.va.gov/guidelines/Pain/cot

32. Nuckols TK, Anderson L, Popescu I, et al. Opioid prescribing: a systematic review and critical appraisal of guidelines for chronic pain. Ann Intern Med 2014;160:38-47.

33. Liu Y, Logan JE, Paulozzi LJ, Zhang K, Jones CM. Potential misuse and inappropriate prescription practices involving opioid analgesics. Am J Manag Care 2013;19:648-65.

34. Mack KA, Zhang K, Paulozzi L, Jones C. Prescription practices involving opioid analgesics among Americans with Medicaid, 2010. J Health Care Poor Underserved 2015;26:182-98. http://dx.doi. org/10.1353/hpu.2015.0009

35. Institute of Medicine. Dying in America: improving quality and honoring individual preferences near the end of life. Washington, DC: The National Academies Press; 2015.

36. Mazer-Amirshahi M, Mullins PM, Rasooly IR, van den Anker J, Pines JM. Trends in prescription opioid use in pediatric emergency department patients. Pediatr Emerg Care 2014;30:230-5. http:// dx.doi.org/10.1097/PEC.0000000000000102 
37. DeVries A, Koch T, Wall E, Getchius T, Chi W, Rosenberg A. Opioid use among adolescent patients treated for headache. J Adolesc Health 2014;55:128-33. http://dx.doi.org/10.1016/j.jadohealth.2013.12.014

38. Veliz P, Epstein-Ngo QM, Meier E, Ross-Durow PL, McCabe SE, Boyd CJ. Painfully obvious: a longitudinal examination of medical use and misuse of opioid medication among adolescent sports participants. J Adolesc Health 2014;54:333-40. http://dx.doi.org/10.1016/j. jadohealth.2013.09.002

39. McCabe SE, West BT, Boyd CJ. Leftover prescription opioids and nonmedical use among high school seniors: a multi-cohort national study. J Adolesc Health 2013;52:480-5. http://dx.doi.org/10.1016/j. jadohealth.2012.08.007

40. McCabe SE, West BT, Boyd CJ. Medical use, medical misuse, and nonmedical use of prescription opioids: results from a longitudinal study. Pain 2013;154:708-13. http://dx.doi.org/10.1016/j.pain.2013.01.011

41. Miech R, Johnston L, O’Malley PM, Keyes KM, Heard K. Prescription opioids in adolescence and future opioid misuse. Pediatrics 2015;136:e1169-77. http://dx.doi.org/10.1542/peds.2015-1364

42. Cerdá M, Santaella J, Marshall BD, Kim JH, Martins SS. Nonmedical prescription opioid use in childhood and early adolescence predicts transitions to heroin use in young adulthood: a national study. J Pediatr 2015;167:605-12.e2. http://dx.doi.org/10.1016/j.jpeds.2015.04.071

43. Cantrill SV, Brown MD, Carlisle RJ, et al.; American College of Emergency Physicians Opioid Guideline Writing Panel. Clinical policy: critical issues in the prescribing of opioids for adult patients in the emergency department. Ann Emerg Med 2012;60:499-525. http:// dx.doi.org/10.1016/j.annemergmed.2012.06.013

44. American Society of Anesthesiologists Task Force on Acute Pain Management. Practice guidelines for acute pain management in the perioperative setting: an updated report by the American Society of Anesthesiologists Task Force on Acute Pain Management. Anesthesiology 2012;116:248-73. http://dx.doi.org/10.1097/ALN.0b013e31823c1030

45. Pennsylvania Department of Health, Department of Drug and Alcohol Programs. Pennsylvania guidelines on the use of opioids in dental practice. Harrisburg, PA: Pennsylvania Department of Drug and Alcohol Programs; 2015. http://www.ddap.pa.gov/Document $\% 20$ Library/Prescriber_Guidelines_Dental.pdf

46. National Heart Lung and Blood Institute. Evidence-based management of sickle cell disease. Expert Panel report. Washington, DC: National Institutes of Health; 2014.

47. Ahmed F. Advisory Committee on Immunization Practices handbook for developing evidence-based recommendations. Version 1.2. Atlanta, GA: US Department of Health and Human Services, CDC; 2013. http:// www.cdc.gov/vaccines/acip/recs/GRADE/about-grade.html\#resources

48. Balshem $\mathrm{H}$, Helfand M, Schünemann HJ, et al. GRADE guidelines: 3. Rating the quality of evidence. J Clin Epidemiol 2011;64:401-6. http://dx.doi.org/10.1016/j.jclinepi.2010.07.015

49. Guyatt GH, Oxman AD, Vist GE, et al.; GRADE Working Group. GRADE: an emerging consensus on rating quality of evidence and strength of recommendations. BMJ 2008;336:924-6. http://dx.doi. org/10.1136/bmj.39489.470347.AD

50. Andrews JC, Schünemann HJ, Oxman AD, et al. GRADE guidelines: 15. Going from evidence to recommendation-determinants of a recommendation's direction and strength. J Clin Epidemiol 2013;66:726-35. http://dx.doi.org/10.1016/j.jclinepi.2013.02.003
51. Guyatt GH, Oxman AD, Schünemann HJ, Tugwell P, Knottnerus A. GRADE guidelines: a new series of articles in the Journal of Clinical Epidemiology. J Clin Epidemiol 2011;64:380-2. http://dx.doi. org/10.1016/j.jclinepi.2010.09.011

52. Chou R, Turner JA, Devine EB, et al. The effectiveness and risks of long-term opioid therapy for chronic pain: a systematic review for a National Institutes of Health Pathways to Prevention Workshop. Ann Intern Med 2015;162:276-86. http://dx.doi.org/10.7326/M14-2559

53. Agency for Healthcare Research and Quality. Methods guide for effectiveness and comparative effectiveness reviews. Effective Health Care Program. AHRQ Publication No. 10(13)-EHC063-EF. Rockville, MD: US Department of Health and Human Services, Agency for Healthcare Research and Quality; 2013. http://www.effectivehealthcare. ahrq.gov

54. Graham E. The effectiveness and risks of long-term opioid treatment of chronic pain. PROSPERO: international prospective register of systematic reviews. York, UK: University of York; 2014. http://www.crd. york.ac.uk/PROSPERO/display_record.asp?ID=CRD42014007016

55. Banta-Green CJ, Merrill JO, Doyle SR, Boudreau DM, Calsyn DA. Opioid use behaviors, mental health and pain-development of a typology of chronic pain patients. Drug Alcohol Depend 2009;104:3442. http://dx.doi.org/10.1016/j.drugalcdep.2009.03.021

56. Boscarino JA, Rukstalis M, Hoffman SN, et al. Risk factors for drug dependence among out-patients on opioid therapy in a large US healthcare system. Addiction 2010;105:1776-82. http://dx.doi. org/10.1111/j.1360-0443.2010.03052.x

57. Compton PA, Wu SM, Schieffer B, Pham Q, Naliboff BD. Introduction of a self-report version of the Prescription Drug Use Questionnaire and relationship to medication agreement noncompliance. J Pain Symptom Manage 2008;36:383-95. http://dx.doi.org/10.1016/j. jpainsymman.2007.11.006

58. Cowan DT, Wilson-Barnett J, Griffiths P, Allan LG. A survey of chronic noncancer pain patients prescribed opioid analgesics. Pain Med 2003;4:340-51. http://dx.doi.org/10.1111/j.1526-4637.2003.03038.x

59. Fleming MF, Balousek SL, Klessig CL, Mundt MP, Brown DD. Substance use disorders in a primary care sample receiving daily opioid therapy. J Pain 2007;8:573-82. http://dx.doi.org/10.1016/j. jpain.2007.02.432

60. Højsted J, Nielsen PR, Guldstrand SK, Frich L, Sjøgren P. Classification and identification of opioid addiction in chronic pain patients. Eur J Pain 2010;14:1014-20. http://dx.doi.org/10.1016/j.ejpain.2010.04.006

61. Portenoy RK, Farrar JT, Backonja MM, et al. Long-term use of controlled-release oxycodone for noncancer pain: results of a 3-year registry study. Clin J Pain 2007;23:287-99. http://dx.doi.org/10.1097/ AJP.0b013e31802b582f

62. Reid MC, Engles-Horton LL, Weber MB, Kerns RD, Rogers EL, O'Connor PG. Use of opioid medications for chronic noncancer pain syndromes in primary care. J Gen Intern Med 2002;17:173-9. http:// dx.doi.org/10.1046/j.1525-1497.2002.10435.x

63. Saffier K, Colombo C, Brown D, Mundt MP, Fleming MF. Addiction Severity Index in a chronic pain sample receiving opioid therapy. J Subst Abuse Treat 2007;33:303-11. http://dx.doi.org/10.1016/j. jsat.2006.12.011

64. Schneider JP, Kirsh KL. Defining clinical issues around tolerance, hyperalgesia, and addiction: a quantitative and qualitative outcome study of long-term opioid dosing in a chronic pain practice. J Opioid Manag 2010;6:385-95. http://dx.doi.org/10.5055/jom.2010.0036 
65. Wasan AD, Butler SF, Budman SH, et al. Does report of craving opioid medication predict aberrant drug behavior among chronic pain patients? Clin J Pain 2009;25:193-8. http://dx.doi.org/10.1097/ AJP.0b013e318193a6c4

66. Dunn KM, Saunders KW, Rutter CM, et al. Opioid prescriptions for chronic pain and overdose: a cohort study. Ann Intern Med 2010;152:8592. http://dx.doi.org/10.7326/0003-4819-152-2-201001190-00006

67. Gomes T, Mamdani MM, Dhalla IA, Paterson JM, Juurlink DN. Opioid dose and drug-related mortality in patients with nonmalignant pain. Arch Intern Med 2011;171:686-91. http://dx.doi.org/10.1001/ archinternmed.2011.117

68. Saunders KW, Dunn KM, Merrill JO, et al. Relationship of opioid use and dosage levels to fractures in older chronic pain patients. J Gen Intern Med 2010;25:310-5. http://dx.doi.org/10.1007/ s11606-009-1218-z

69. Li L, Setoguchi S, Cabral H, Jick S. Opioid use for noncancer pain and risk of fracture in adults: a nested case-control study using the general practice research database. Am J Epidemiol 2013;178:559-69. http://dx.doi.org/10.1093/aje/kwt013

70. Carman WJ, Su S, Cook SF, Wurzelmann JI, McAfee A. Coronary heart disease outcomes among chronic opioid and cyclooxygenase- 2 users compared with a general population cohort. Pharmacoepidemiol Drug Saf 2011;20:754-62. http://dx.doi.org/10.1002/pds.2131

71. Li L, Setoguchi S, Cabral H, Jick S. Opioid use for noncancer pain and risk of myocardial infarction amongst adults. J Intern Med 2013;273:511-26. http://dx.doi.org/10.1111/joim.12035

72. Deyo RA, Smith DH, Johnson ES, et al. Prescription opioids for back pain and use of medications for erectile dysfunction. Spine (Phila Pa 1976) 2013;38:909-15. http://dx.doi.org/10.1097/BRS.0b013e3182830482

73. Rubinstein A, Carpenter DM. Elucidating risk factors for androgen deficiency associated with daily opioid use. Am J Med 2014;127:1195201. http://dx.doi.org/10.1016/j.amjmed.2014.07.015

74. Gomes T, Redelmeier DA, Juurlink DN, Dhalla IA, Camacho X, Mamdani MM. Opioid dose and risk of road trauma in Canada: a population-based study. JAMA Intern Med 2013;173:196-201. http:// dx.doi.org/10.1001/2013.jamainternmed.733

75. Salzman RT, Roberts MS, Wild J, Fabian C, Reder RF, Goldenheim PD. Can a controlled-release oral dose form of oxycodone be used as readily as an immediate-release form for the purpose of titrating to stable pain control? J Pain Symptom Manage 1999;18:271-9. http:// dx.doi.org/10.1016/S0885-3924(99)00079-2

76. Jamison RN, Raymond SA, Slawsby EA, Nedeljkovic SS, Katz NP. Opioid therapy for chronic noncancer back pain. A randomized prospective study. Spine (Phila Pa 1976) 1998;23:2591-600. http:// dx.doi.org/10.1097/00007632-199812010-00014

77. Miller M, Barber CW, Leatherman S, et al. Prescription opioid duration of action and the risk of unintentional overdose among patients receiving opioid therapy. JAMA Intern Med 2015;175:608-15. http:// dx.doi.org/10.1001/jamainternmed.2014.8071

78. Allan L, Richarz U, Simpson K, Slappendel R. Transdermal fentanyl versus sustained release oral morphine in strong-opioid naïve patients with chronic low back pain. Spine (Phila Pa 1976) 2005;30:2484-90. http://dx.doi.org/10.1097/01.brs.0000186860.23078.a8

79. Wild JE, Grond S, Kuperwasser B, et al. Long-term safety and tolerability of tapentadol extended release for the management of chronic low back pain or osteoarthritis pain. Pain Pract 2010;10:41627. http://dx.doi.org/10.1111/j.1533-2500.2010.00397.x
80. Mitra F, Chowdhury S, Shelley M, Williams G. A feasibility study of transdermal buprenorphine versus transdermal fentanyl in the longterm management of persistent non-cancer pain. Pain Med 2013;14:75-83. http://dx.doi.org/10.1111/pme.12011

81. Krebs EE, Becker WC, Zerzan J, Bair MJ, McCoy K, Hui S. Comparative mortality among Department of Veterans Affairs patients prescribed methadone or long-acting morphine for chronic pain. Pain 2011;152:1789-95. http://dx.doi.org/10.1016/j.pain.2011.03.023

82. Hartung DM, Middleton L, Haxby DG, Koder M, Ketchum KL, Chou R. Rates of adverse events of long-acting opioids in a state Medicaid program. Ann Pharmacother 2007;41:921-8. http://dx.doi. org/10.1345/aph.1K066

83. Ray WA, Chung CP, Murray KT, Cooper WO, Hall K, Stein CM. Out-of-hospital mortality among patients receiving methadone for noncancer pain. JAMA Intern Med 2015;175:420-7. http://dx.doi. org/10.1001/jamainternmed.2014.6294

84. Naliboff BD, Wu SM, Schieffer B, et al. A randomized trial of 2 prescription strategies for opioid treatment of chronic nonmalignant pain. J Pain 2011;12:288-96. http://dx.doi.org/10.1016/j. jpain.2010.09.003

85. Cowan DT, Wilson-Barnett J, Griffiths P, Vaughan DJ, Gondhia A, Allan LG. A randomized, double-blind, placebo-controlled, cross-over pilot study to assess the effects of long-term opioid drug consumption and subsequent abstinence in chronic noncancer pain patients receiving controlled-release morphine. Pain Med 2005;6:113-21. http://dx.doi. org/10.1111/j.1526-4637.2005.05020.x

86. Ralphs JA, Williams AC, Richardson PH, Pither CE, Nicholas MK. Opiate reduction in chronic pain patients: a comparison of patientcontrolled reduction and staff controlled cocktail methods. Pain 1994;56:279-88. http://dx.doi.org/10.1016/0304-3959(94)90166-X

87. Tennant FS Jr, Rawson RA. Outpatient treatment of prescription opioid dependence: comparison of two methods. Arch Intern Med 1982;142:1845-7. http://dx.doi.org/10.1001/archinte.1982.00340230087016

88. Akbik H, Butler SF, Budman SH, Fernandez K, Katz NP, Jamison RN. Validation and clinical application of the Screener and Opioid Assessment for Patients with Pain (SOAPP). J Pain Symptom Manage 2006;32:287-93. http://dx.doi.org/10.1016/j.jpainsymman.2006.03.010

89. Jones T, Moore T, Levy JL, et al. A comparison of various risk screening methods in predicting discharge from opioid treatment. Clin J Pain 2012;28:93-100. http://dx.doi.org/10.1097/AJP.0b013e318225da9e

90. Moore TM, Jones T, Browder JH, Daffron S, Passik SD. A comparison of common screening methods for predicting aberrant drug-related behavior among patients receiving opioids for chronic pain management. Pain Med 2009;10:1426-33. http://dx.doi. org/10.1111/j.1526-4637.2009.00743.x

91. Webster LR, Webster RM. Predicting aberrant behaviors in opioidtreated patients: preliminary validation of the Opioid Risk Tool. Pain Med 2005;6:432-42. http://dx.doi.org/10.1111/j.1526-4637.2005.00072.x

92. Jones T, Lookatch S, Grant P, McIntyre J, Moore T. Further validation of an opioid risk assessment tool: the Brief Risk Interview. J Opioid Manag 2014;10:353-64. http://dx.doi.org/10.5055/jom.2014.0226

93. Jones T, Moore T. Preliminary data on a new opioid risk assessment measure: the Brief Risk Interview. J Opioid Manag 2013;9:19-27. http://dx.doi.org/10.5055/jom.2013.0143

94. Alam A, Gomes T, Zheng H, Mamdani MM, Juurlink DN, Bell CM. Long-term analgesic use after low-risk surgery: a retrospective cohort study. Arch Intern Med 2012;172:425-30. http://dx.doi.org/10.1001/ archinternmed.2011.1827 
95. Webster BS, Verma SK, Gatchel RJ. Relationship between early opioid prescribing for acute occupational low back pain and disability duration, medical costs, subsequent surgery and late opioid use. Spine (Phila Pa 1976) 2007;32:2127-32. http://dx.doi.org/10.1097/BRS.0b013e318145a731

96. Ganann R, Ciliska D, Thomas H. Expediting systematic reviews: methods and implications of rapid reviews. Implement Sci 2010;5:56. http://dx.doi.org/10.1186/1748-5908-5-56

97. Williams AC, Eccleston C, Morley S. Psychological therapies for the management of chronic pain (excluding headache) in adults. Cochrane Database Syst Rev 2012;11:CD007407.

98. Hayden JA, van Tulder MW, Malmivaara A, Koes BW. Exercise therapy for treatment of non-specific low back pain. Cochrane Database Syst Rev 2005;3:CD000335.

99. Fransen M, McConnell S, Harmer AR, Van der Esch M, Simic M, Bennell KL. Exercise for osteoarthritis of the knee. Cochrane Database Syst Rev 2015;1:CD004376.

100. Fransen M, McConnell S, Hernandez-Molina G, Reichenbach S. Exercise for osteoarthritis of the hip. Cochrane Database Syst Rev 2014;4:CD007912 10.1002/14651858.

101. Busch AJ, Barber KA, Overend TJ, Peloso PM, Schachter CL. Exercise for treating fibromyalgia syndrome. Cochrane Database Syst Rev 2007;4:CD003786.

102. Lee C, Crawford C, Swann S; Active Self-Care Therapies for Pain (PACT) Working Group. Multimodal, integrative therapies for the self-management of chronic pain symptoms. Pain Med 2014;15(Suppl 1): S76-85. http://dx.doi.org/10.1111/pme.12408

103. Kamper SJ, Apeldoorn AT, Chiarotto A, et al. Multidisciplinary biopsychosocial rehabilitation for chronic low back pain: Cochrane systematic review and meta-analysis. BMJ 2015;350:h444. http:// dx.doi.org/10.1136/bmj.h444

104. Zhang W, Doherty M, Arden N, et al.; EULAR Standing Committee for International Clinical Studies Including Therapeutics (ESCISIT). EULAR evidence based recommendations for the management of hip osteoarthritis: report of a task force of the EULAR Standing Committee for International Clinical Studies Including Therapeutics (ESCISIT). Ann Rheum Dis 2005;64:669-81. http://dx.doi.org/10.1136/ ard.2004.028886

105. Zhang W, Doherty M, Leeb BF, et al. EULAR evidence based recommendations for the management of hand osteoarthritis: report of a Task Force of the EULAR Standing Committee for International Clinical Studies Including Therapeutics (ESCISIT). Ann Rheum Dis 2007;66:377-88. http://dx.doi.org/10.1136/ard.2006.062091

106. Zhang W, Moskowitz RW, Nuki G, et al. OARSI recommendations for the management of hip and knee osteoarthritis, Part II: OARSI evidence-based, expert consensus guidelines. Osteoarthritis Cartilage 2008;16:137-62. http://dx.doi.org/10.1016/j.joca.2007.12.013

107. Zhang W, Moskowitz RW, Nuki G, et al. OARSI recommendations for the management of hip and knee osteoarthritis, part I: critical appraisal of existing treatment guidelines and systematic review of current research evidence. Osteoarthritis Cartilage 2007;15:981-1000. http://dx.doi.org/10.1016/j.joca.2007.06.014

108. Jordan KM, Arden NK, Doherty M, et al.; Standing Committee for International Clinical Studies Including Therapeutic Trials ESCISIT. EULAR recommendations 2003: an evidence based approach to the management of knee osteoarthritis: report of a Task Force of the Standing Committee for International Clinical Studies Including Therapeutic Trials (ESCISIT). Ann Rheum Dis 2003;62:1145-55. http://dx.doi.org/10.1136/ard.2003.011742
109. American Geriatrics Society Panel on Pharmacological Management of Persistent Pain in Older Persons. Pharmacological management of persistent pain in older persons. J Am Geriatr Soc 2009;57:1331-46. http://dx.doi.org/10.1111/j.1532-5415.2009.02376.x

110. Chou R, Qaseem A, Snow V, et al.; Clinical Efficacy Assessment Subcommittee of the American College of Physicians; American College of Physicians; American Pain Society Low Back Pain Guidelines Panel. Diagnosis and treatment of low back pain: a joint clinical practice guideline from the American College of Physicians and the American Pain Society. Ann Intern Med 2007;147:478-91. http://dx.doi. org/10.7326/0003-4819-147-7-200710020-00006

111. Trelle S, Reichenbach S, Wandel S, et al. Cardiovascular safety of nonsteroidal anti-inflammatory drugs: network meta-analysis. BMJ 2011;342:c7086. http://dx.doi.org/10.1136/bmj.c7086

112. Food and Drug Administration. FDA drug safety communication: FDA strengthens warning that non-aspirin nonsteroidal antiinflammatory drugs (NSAIDs) can cause heart attacks or strokes. Silver Spring, MD: US Department of Health and Human Services, Food and Drug Administration; 2015. http://www.fda.gov/Drugs/ DrugSafety/ucm 451800.htm

113. O'Connor AB, Dworkin RH. Treatment of neuropathic pain: an overview of recent guidelines. Am J Med 2009;122(Suppl):S22-32. http://dx.doi.org/10.1016/j.amjmed.2009.04.007

114. Attal N, Cruccu G, Baron R, et al.; European Federation of Neurological Societies. EFNS guidelines on the pharmacological treatment of neuropathic pain: 2010 revision. Eur J Neurol 2010;17:1113-e88. http://dx.doi.org/10.1111/j.1468-1331.2010.02999.x

115. Moulin DE, Clark AJ, Gilron I, et al.; Canadian Pain Society. Pharmacological management of chronic neuropathic pain - consensus statement and guidelines from the Canadian Pain Society. Pain Res Manag 2007;12:13-21.

116. Bril V, England J, Franklin GM, et al.; American Academy of Neurology; American Association of Neuromuscular and Electrodiagnostic Medicine; American Academy of Physical Medicine and Rehabilitation. Evidence-based guideline: Treatment of painful diabetic neuropathy: report of the American Academy of Neurology, the American Association of Neuromuscular and Electrodiagnostic Medicine, and the American Academy of Physical Medicine and Rehabilitation. Neurology 2011;76:1758-65. Corrected in: Neurology 2011;77:603. http://dx.doi.org/10.1212/WNL.0b013e3182166ebe

117. Wallen M, Gillies D. Intra-articular steroids and splints/rest for children with juvenile idiopathic arthritis and adults with rheumatoid arthritis. Cochrane Database Syst Rev 2006:(1):CD002824.

118. Bellamy N, Campbell J, Robinson V, Gee T, Bourne R, Wells G. Intraarticular corticosteroid for treatment of osteoarthritis of the knee. Cochrane Database Syst Rev 2006;2:CD005328.

119. Buchbinder R, Green S, Youd JM. Corticosteroid injections for shoulder pain. Cochrane Database Syst Rev 2003;1:CD004016.

120. Food and Drug Administration. Epidural corticosteroid injection: drug safety communication. Silver Spring, MD: US Department of Health and Human Services, Food and Drug Administration; 2014. http:// www.fda.gov/Safety/Med Watch/SafetyInformation/ SafetyAlertsforHumanMedicalProducts/ucm394530.htm

121. Goal of label changes: better prescribing, safer use of opioids. Silver Spring, MD: US Department of Health and Human Services, Food and Drug Administration; 2013. http://www.fda.gov/ForConsumers/ ConsumerUpdates/ucm367660.htm 
122. Von Korff M, Merrill JO, Rutter CM, Sullivan M, Campbell CI, Weisner C. Time-scheduled vs. pain-contingent opioid dosing in chronic opioid therapy. Pain 2011;152:1256-62. http://dx.doi. org/10.1016/j.pain.2011.01.005

123. Paulozzi L, Mack KA, Jones CM. Vital signs: risk for overdose from methadone used for pain relief-United States, 1999-2010. MMWR Morb Mortal Wkly Rep 2012;61:493-7.

124. Gwira Baumblatt JA, Wiedeman C, Dunn JR, Schaffner W, Paulozzi LJ, Jones TF. High-risk use by patients prescribed opioids for pain and its role in overdose deaths. JAMA Intern Med 2014;174:796-801. http://dx.doi.org/10.1001/jamainternmed.2013.12711

125. Paulozzi LJ, Kilbourne EM, Shah NG, et al. A history of being prescribed controlled substances and risk of drug overdose death. Pain Med 2012;13:87-95. http://dx.doi.org/10.1111/j.1526-4637.2011.01260.x

126. Liang Y, Turner BJ. Assessing risk for drug overdose in a national cohort: role for both daily and total opioid dose? J Pain 2015;16:318-25. http://dx.doi.org/10.1016/j.jpain.2014.11.007

127. Bohnert ASB, Logan JE, Ganoczy D, Dowell D. A detailed exploration into the association of prescribed opioid dosage and prescription opioid overdose deaths among patients with chronic pain. Med Care 2016. Epub ahead of print. http://journals.lww.com/lww-medicalcare/ Abstract/publishahead/A_Detailed_Exploration_Into_the_ Association_of.98952.aspx

128. Dasgupta N, Funk MJ, Proescholdbell S, Hirsch A, Ribisl KM, Marshall S. Cohort study of the impact of high-dose opioid analgesics on overdose mortality. Pain Med 2015. Epub ahead of print. https://uncch.pure.elsevier.com/en/publications/ cohort-study-of-the-impact-of-high-dose-opioid-analgesics-on-over

129. Jones CM, McAninch JK. Emergency department visits and overdose deaths from combined use of opioids and benzodiazepines. Am J Prev Med 2015;49:493-501. http://dx.doi.org/10.1016/j.amepre.2015.03.040

130. Ballantyne JC, Mao J. Opioid therapy for chronic pain. N Engl J Med 2003;349:1943-53. http://dx.doi.org/10.1056/NEJMra025411

131. Kalso E, Simpson KH, Slappendel R, Dejonckheere J, Richarz U. Predicting long-term response to strong opioids in patients with low back pain: findings from a randomized, controlled trial of transdermal fentanyl and morphine. BMC Med 2007;5:39. http://dx.doi. org/10.1186/1741-7015-5-39

132. Yue HJ, Guilleminault C. Opioid medication and sleep-disordered breathing. Med Clin North Am 2010;94:435-46. http://dx.doi. org/10.1016/j.mcna.2010.02.007

133. Webster LR, Choi Y, Desai H, Webster L, Grant BJB. Sleep-disordered breathing and chronic opioid therapy. Pain Med 2008;9:425-32. http:// dx.doi.org/10.1111/j.1526-4637.2007.00343.x

134. Goodman LS, Limberd LE. Goodman and Gilman's the pharmacologic basis of therapeutics. 9th ed. New York, NY: McGraw-Hill; 1996.

135. Rowe JW, Andres R, Tobin JD, Norris AH, Shock NW. The effect of age on creatinine clearance in men: a cross-sectional and longitudinal study. J Gerontol 1976;31:155-63. http://dx.doi.org/10.1093/ geronj/31.2.155

136. Rolita L, Spegman A, Tang X, Cronstein BN. Greater number of narcotic analgesic prescriptions for osteoarthritis is associated with falls and fractures in elderly adults. J Am Geriatr Soc 2013;61:335-40. http://dx.doi.org/10.1111/jgs.12148

137. Vestergaard P, Rejnmark L, Mosekilde L. Fracture risk associated with the use of morphine and opiates. J Intern Med 2006;260:76-87. http:// dx.doi.org/10.1111/j.1365-2796.2006.01667.x
138. Spector W, Shaffer T, Potter DE, Correa-de-Araujo R, Rhona Limcangco M. Risk factors associated with the occurrence of fractures in U.S. nursing homes: resident and facility characteristics and prescription medications. J Am Geriatr Soc 2007;55:327-33. http:// dx.doi.org/10.1111/j.1532-5415.2007.01081.x

139. Yazdy MM, Mitchell AA, Tinker SC, Parker SE, Werler MM. Periconceptional use of opioids and the risk of neural tube defects. Obstet Gynecol 2013;122:838-44. http://dx.doi.org/10.1097/ AOG.0b013e3182a6643c

140. Broussard CS, Rasmussen SA, Reefhuis J, et al. Maternal treatment with opioid analgesics and risk for birth defects. Am J Obstet Gynecol 2011;204:314 e1-11.

141. Whiteman VE, Salemi JL, Mogos MF, Cain MA, Aliyu MH, Salihu HM. Maternal opioid drug use during pregnancy and its impact on perinatal morbidity, mortality, and the costs of medical care in the United States. J Pregnancy 2014;906723 10.1155/2014/906723.

142. Hadi I, da Silva O, Natale R, Boyd D, Morley-Forster PK. Opioids in the parturient with chronic nonmalignant pain: a retrospective review. J Opioid Manag 2006;2:31-4.

143. Edlund MJ, Steffick D, Hudson T, Harris KM, Sullivan M. Risk factors for clinically recognized opioid abuse and dependence among veterans using opioids for chronic non-cancer pain. Pain 2007;129:355-62. http://dx.doi.org/10.1016/j.pain.2007.02.014

144. Howe CQ, Sullivan MD. The missing 'P' in pain management: how the current opioid epidemic highlights the need for psychiatric services in chronic pain care. Gen Hosp Psychiatry 2014;36:99-104. http:// dx.doi.org/10.1016/j.genhosppsych.2013.10.003

145. Turner BJ, Liang Y. Drug Overdose in a retrospective cohort with non-cancer pain treated with opioids, antidepressants, and/or sedativehypnotics: interactions with mental health disorders. J Gen Intern Med 2015;30:1081-96; Epub ahead of print. http://dx.doi.org/10.1007/ s11606-015-3199-4

146. Paulozzi L. CDC grand rounds: prescription drug overdoses-a U.S. epidemic. MMWR Morb Mortal Wkly Rep 2012;61:10-3.

147. Walley AY, Xuan Z, Hackman HH, et al. Opioid overdose rates and implementation of overdose education and nasal naloxone distribution in Massachusetts: interrupted time series analysis. BMJ 2013;346:f174. http://dx.doi.org/10.1136/bmj.f174

148. Coffin P, Banta-Green C. The dueling obligations of opioid stewardship. Ann Intern Med 2014;160:207-8. http://dx.doi.org/10.7326/ M13-2781

149. Twillman RK, Kirch R, Gilson A. Efforts to control prescription drug abuse: Why clinicians should be concerned and take action as essential advocates for rational policy. CA Cancer J Clin 2014;64:369-76. http:// dx.doi.org/10.3322/caac. 21243

150. Cicero TJ, Ellis MS, Surratt HL. Effect of abuse-deterrent formulation of OxyContin. N Engl J Med 2012;367:187-9. http://dx.doi. org/10.1056/NEJMc1204141

151. Mattick RP, Breen C, Kimber J, Davoli M. Buprenorphine maintenance versus placebo or methadone maintenance for opioid dependence. Cochrane Database Syst Rev 2014;2:CD002207 10.1002/14651858.

152. Mattick RP, Breen C, Kimber J, Davoli M. Methadone maintenance therapy versus no opioid replacement therapy for opioid dependence. Cochrane Database Syst Rev 2009;3:CD002209 10.1002/14651858.

153. Fullerton CA, Kim M, Thomas CP, et al. Medication-assisted treatment with methadone: assessing the evidence. Psychiatr Serv 2014;65:14657. http://dx.doi.org/10.1176/appi.ps.201300235 
154. Amato L, Minozzi S, Davoli M, Vecchi S. Psychosocial and pharmacological treatments versus pharmacological treatments for opioid detoxification. Cochrane Database Syst Rev 2011;9:CD005031 $10.1002 / 14651858$.

155. Connock M, Juarez-Garcia A, Jowett S, et al. Methadone and buprenorphine for the management of opioid dependence: a systematic review and economic evaluation. Health Technol Assess 2007;11:1-171. http://dx.doi.org/10.3310/hta11090

156. Keller CE, Ashrafioun L, Neumann AM, Van Klein J, Fox CH, Blondell RD. Practices, perceptions, and concerns of primary care physicians about opioid dependence associated with the treatment of chronic pain. Subst Abus 2012;33:103-13. http://dx.doi.org/10.1080/08897077.2 011.630944

157. Payne M, Gething M, Moore AA, Reid MC. Primary care providers' perspectives on psychoactive medication disorders in older adults. Am J Geriatr Pharmacother 2011;9:164-72. http://dx.doi.org/10.1016/j. amjopharm.2011.04.004

158. Hagemeier NE, Gray JA, Pack RP. Prescription drug abuse: a comparison of prescriber and pharmacist perspectives. Subst Use Misuse 2013;48:761-8. http://dx.doi.org/10.3109/10826084.2013.787101

159. Hooten WM, Bruce BK. Beliefs and attitudes about prescribing opioids among healthcare providers seeking continuing medical education. J Opioid Manag 2011;7:417-24.

160. Hwang CS, Turner LW, Kruszewski SP, Kolodny A, Alexander GC. Prescription drug abuse: a national survey of primary care physicians. JAMA Intern Med 2015;175:302-4. http://dx.doi.org/10.1001/ jamainternmed.2014.6520

161. Green TC, Mann MR, Bowman SE, et al. How does use of a prescription monitoring program change medical practice? Pain Med 2012;13:131423. http://dx.doi.org/10.1111/j.1526-4637.2012.01452.x

162. Ringwalt C, Garrettson M, Alexandridis A. The effects of North Carolina's prescription drug monitoring program on the prescribing behaviors of the state's providers. J Prim Prev 2015;36:131-7. http:// dx.doi.org/10.1007/s10935-014-0381-0

163. Pergolizzi J, Pappagallo M, Stauffer J, et al.; Integrated Drug Compliance Study Group (IDCSG). The role of urine drug testing for patients on opioid therapy. Pain Pract 2010;10:497-507. http://dx.doi. org/10.1111/j.1533-2500.2010.00375.x

164. Starrels JL, Wu B, Peyser D, et al. It made my life a little easier: primary care providers' beliefs and attitudes about using opioid treatment agreements. J Opioid Manag 2014;10:95-102. http://dx.doi. org/10.5055/jom.2014.0198

165. Smith RJ, Kilaru AS, Perrone J, et al. How, why, and for whom do emergency medicine providers use prescription drug monitoring programs? Pain Med 2015;16:1122-31. http://dx.doi.org/10.1111/ pme. 12700

166. Bair MJ, Krebs EE. Why is urine drug testing not used more often in practice? Pain Pract 2010;10:493-6. http://dx.doi.org/10.1111/ j.1533-2500.2010.00425.x

167. Mangione MP, Crowley-Matoka M. Improving pain management communication: how patients understand the terms "opioid" and "narcotic". J Gen Intern Med 2008;23:1336-8. http://dx.doi. org/10.1007/s11606-008-0658-1

168. Moore SK, Guarino H, Acosta MC, et al. Patients as collaborators: using focus groups and feedback sessions to develop an interactive, web-based self-management intervention for chronic pain. Pain Med 2013;14:1730-40. http://dx.doi.org/10.1111/pme.12200
169. Simmonds MJ, Finley EP, Vale S, Pugh MJ, Turner BJ. A qualitative study of veterans on long-term opioid analgesics: barriers and facilitators to multimodality pain management. Pain Med 2015;16:726-32. http:// dx.doi.org/10.1111/pme.12626

170. Hansen RN, Oster G, Edelsberg J, Woody GE, Sullivan SD. Economic costs of nonmedical use of prescription opioids. Clin J Pain 2011;27:194-202. http://dx.doi.org/10.1097/AJP.0b013e3181ff04ca

171. Birnbaum HG, White AG, Schiller M, Waldman T, Cleveland JM, Roland CL. Societal costs of prescription opioid abuse, dependence, and misuse in the United States. Pain Med 2011;12:657-67. http:// dx.doi.org/10.1111/j.1526-4637.2011.01075.x

172. Inocencio TJ, Carroll NV, Read EJ, Holdford DA. The economic burden of opioid-related poisoning in the United States. Pain Med 2013;14:1534-47. http://dx.doi.org/10.1111/pme.12183

173. Stagnitti MN. Trends in prescribed outpatient opioid use and expenses in the U.S. civilian noninstitutionalized population, 2002-2012. Statistical Brief No. 478. Rockville, MD: Agency for Healthcare Research and Quality; 2015.

174. Gore M, Tai K-S, Sadosky A, Leslie D, Stacey BR. Use and costs of prescription medications and alternative treatments in patients with osteoarthritis and chronic low back pain in community-based settings. Pain Pract 2012;12:550 60. http://dx.doi.org/10.1111/j.1533-2500.2012.00532.x

175. Laffer A, Murphy R, Winegarden W, et al. An economic analysis of the costs and benefits associated with regular urine drug testing for chronic pain patients in the United States. Nashville, TN: Laffer Associates; 2011.

176. Hochberg MC, Altman RD, April KT, et al.; American College of Rheumatology. American College of Rheumatology 2012 recommendations for the use of nonpharmacologic and pharmacologic therapies in osteoarthritis of the hand, hip, and knee. Arthritis Care Res (Hoboken) 2012;64:465-74. http://dx.doi.org/10.1002/acr.21596

177. Sullivan MD, Edlund MJ, Zhang L, Unützer J, Wells KB. Association between mental health disorders, problem drug use, and regular prescription opioid use. Arch Intern Med 2006;166:2087-93. http:// dx.doi.org/10.1001/archinte.166.19.2087

178. Jones CM, Mack KA, Paulozzi LJ. Pharmaceutical overdose deaths, United States, 2010. JAMA 2013;309:657-9. http://dx.doi. org/10.1001/jama.2013.272

179. Hooten WM, Timming R, Belgrade M, et al. Assessment and management of chronic pain. Bloomington, MN: Institute for Clinical Systems Improvement; 2013. https:/www.icsi.org/_asset/bw798b/ ChronicPain.pdf

180. The Interagency Pain Research Coordinating Committee. Draft of the National Pain Strategy: a comprehensive population health level strategy for pain. Bethesda, MD: National Institutes of Health; 2015. http:// iprcc.nih.gov/National_Pain_Strategy/NPS_Main.htm

181. Mannion AF, Müntener M, Taimela S, Dvorak J. A randomized clinical trial of three active therapies for chronic low back pain. Spine (Phila Pa 1976) 1999;24:2435-48. http://dx.doi.org/10.1097/00007632-199912010-00004

182. Chaparro LE, Furlan AD, Deshpande A, Mailis-Gagnon A, Atlas S, Turk DC. Opioids compared with placebo or other treatments for chronic low back pain: an update of the Cochrane Review. Spine (Phila Pa 1976) 2014;39:556-63. http://dx.doi.org/10.1097/BRS.0000000000000249

183. Loder E, Weizenbaum E, Frishberg B, Silberstein S; American Headache Society Choosing Wisely Task Force. Choosing wisely in headache medicine: the American Headache Society's list of five things physicians and patients should question. Headache 2013;53:1651-9. http:// dx.doi.org/10.1111/head.12233 
184. Gaskell H, Moore RA, Derry S, Stannard C. Oxycodone for neuropathic pain and fibromyalgia in adults. Cochrane Database Syst Rev 2014;6:CD010692 10.1002/14651858.

185. Noble M, Treadwell JR, Tregear SJ, et al. Long-term opioid management for chronic noncancer pain. Cochrane Database Syst Rev 2010:1:CD006605 10.1002/14651858.CD006605.pub2.

186. Krebs EE, Lorenz KA, Bair MJ, et al. Development and initial validation of the PEG, a three-item scale assessing pain intensity and interference. J Gen Intern Med 2009;24:733-8. http://dx.doi.org/10.1007/ s11606-009-0981-1

187. Ostelo RW, Deyo RA, Stratford P, et al. Interpreting change scores for pain and functional status in low back pain: towards international consensus regarding minimal important change. Spine (Phila Pa 1976) 2008;33:90-4. http://dx.doi.org/10.1097/BRS.0b013e31815e3a10

188. Food and Drug Administration. Disposal of unused medicines: what you should know. Silver Spring, MD: US Department of Health and Human Services, Food and Drug Administration; 2015. http://www.fda.gov/ Drugs/ResourcesForYou/Consumers/BuyingUsingMedicineSafely/ EnsuringSafeUseofMedicine/SafeDisposalofMedicines/ucm 186187. htm\#Flush_List

189. Food and Drug Administration. FDA blueprint for prescriber education for extended-release and long-acting opioid analgesics. Silver Spring, MD: US Department of Health and Human Services, Food and Drug Administration; 2014.

190. Food and Drug Administration. Abuse-deterrent opioids: evaluation and labeling guidance for industry. Silver Spring, MD: US Department of Health and Human Services, Food and Drug Administration, Center for Drug Evaluation and Research; 2015.

191. Chou R, Cruciani RA, Fiellin DA, et al.; American Pain Society; Heart Rhythm Society. Methadone safety: a clinical practice guideline from the American Pain Society and College on Problems of Drug Dependence, in collaboration with the Heart Rhythm Society. J Pain 2014;15:321-37. http://dx.doi.org/10.1016/j.jpain.2014.01.494

192. Chu J, Farmer B, Ginsburg B, Hernandez S, Kenny J, Majlesi N. New York City emergency department discharge opioid prescribing guidelines. New York, NY: New York City Department of Health and Mental Hygiene; 2013. http://www.nyc.gov/html/doh/html/hcp/drugopioid-guidelines.shtml

193. Cheng D, Majlesi N. Clinical practice statement: emergency department opioid prescribing guidelines for the treatment of noncancer related pain. Milwaukee, WI: American Academy of Emergency Medicine; 2013.

194. American College of Emergency Physicians. Maryland emergency department and acute care facility guidelines for prescribing opioids. Baltimore, MD: Maryland Chapter, American College of Emergency Physicians; 2014. http://www.mdacep.org/MD\%20ACEP\%20 Pamphlet\%20FINAL_April\%202014.pdf

195. Paone D, Dowell D, Heller D. Preventing misuse of prescription opioid drugs. City Health Information 2011;30:23-30.

196. Thorson D, Biewen P, Bonte B, et al. Acute pain assessment and opioid prescribing protocol. Bloomington, $\mathrm{MN}$ : Institute for Clinical Systems Improvement; 2014. https:/www.icsi.org/_asset/dyp5wm/Opioids.pdf

197. Cantrill SV, Brown MD, Carlisle RJ, et al.; American College of Emergency Physicians Opioid Guideline Writing Panel. Clinical policy: critical issues in the prescribing of opioids for adult patients in the emergency department. Ann Emerg Med 2012;60:499-525. http:// dx.doi.org/10.1016/j.annemergmed.2012.06.013
198. Coste J, Delecoeuillerie G, Cohen de Lara A, Le Parc JM, Paolaggi JB. Clinical course and prognostic factors in acute low back pain: an inception cohort study in primary care practice. BMJ 1994;308:57780. http://dx.doi.org/10.1136/bmj.308.6928.577

199. CDC. Common elements in guidelines for prescribing opioids for chronic pain. Atlanta, GA: US Department of Health and Human Services, CDC; 2015. http://www.cdc.gov/drugoverdose/prescribing/ common-elements.html

200. Berlin D, Farmer BM, Rao RB, et al. Deaths and severe adverse events associated with anesthesia-assisted rapid opioid detoxification-New York City, 2012. MMWR Morb Mortal Wkly Rep 2013;62:777-80.

201. Berna C, Kulich RJ, Rathmell JP. Tapering long-term opioid therapy in chronic noncancer pain: Evidence and recommendations for everyday practice. Mayo Clin Proc 2015;90:828-42. http://dx.doi.org/10.1016/j. mayocp.2015.04.003

202. ACOG Committee on Health Care for Underserved Women; American Society of Addiction Medicine. ACOG Committee Opinion No. 524: Opioid abuse, dependence, and addiction in pregnancy. Obstet Gynecol 2012;119:1070-6. http://dx.doi.org/10.1097/AOG.0b013e318256496e

203. National Opioid Use Guideline Group. Canadian guideline for safe and effective use of opioids for chronic non-cancer pain; 2010. http:// nationalpaincentre.mcmaster.ca/opioid/documents.html

204. Bernabei R, Gambassi G, Lapane K, et al. Management of pain in elderly patients with cancer. SAGE Study Group. Systematic Assessment of Geriatric Drug Use via Epidemiology. JAMA 1998;279:1877-82. http://dx.doi.org/10.1001/jama.279.23.1877

205. Kroenke K, Spitzer RL, Williams JBBL, Löwe B. The Patient Health Questionnaire Somatic, Anxiety, and Depressive Symptom Scales: a systematic review. Gen Hosp Psychiatry 2010;32:345-59. http://dx.doi. org/10.1016/j.genhosppsych.2010.03.006

206. Saitz R, Cheng DM, Allensworth-Davies D, Winter MR, Smith PC. The ability of single screening questions for unhealthy alcohol and other drug use to identify substance dependence in primary care. J Stud Alcohol Drugs 2014;75:153-7. http://dx.doi.org/10.15288/ jsad.2014.75.153

207. Smith PC, Schmidt SM, Allensworth-Davies D, Saitz R. A singlequestion screening test for drug use in primary care. Arch Intern Med 2010;170:1155-60. http://dx.doi.org/10.1001/archinternmed.2010.140

208. Yudko E, Lozhkina O, Fouts A. A comprehensive review of the psychometric properties of the Drug Abuse Screening Test. J Subst Abuse Treat 2007;32:189-98. http://dx.doi.org/10.1016/j.jsat.2006.08.002

209. Reinert DF, Allen JP. The alcohol use disorders identification test: an update of research findings. Alcohol Clin Exp Res 2007;31:185-99. http://dx.doi.org/10.1111/j.1530-0277.2006.00295.x

210. Enteen L, Bauer J, McLean R, et al. Overdose prevention and naloxone prescription for opioid users in San Francisco. J Urban Health 2010;87:931-41. http://dx.doi.org/10.1007/s11524-010-9495-8

211. Irvine JM, Hallvik SE, Hildebran C, Marino M, Beran T, Deyo RA. Who uses a prescription drug monitoring program and how? Insights from a statewide survey of Oregon clinicians. J Pain 2014;15:747-55. http://dx.doi.org/10.1016/j.jpain.2014.04.003

212. Park TW, Saitz R, Ganoczy D, Ilgen MA, Bohnert AS. Benzodiazepine prescribing patterns and deaths from drug overdose among US veterans receiving opioid analgesics: case-cohort study. BMJ 2015;350:h2698. http://dx.doi.org/10.1136/bmj.h2698

213. Paquin AM, Zimmerman K, Rudolph JL. Risk versus risk: a review of benzodiazepine reduction in older adults. Expert Opin Drug Saf 2014;13:919-34. http://dx.doi.org/10.1517/14740338.2014.925444 
214. Schweizer E, Case WG, Rickels K. Benzodiazepine dependence and withdrawal in elderly patients. Am J Psychiatry 1989;146:529-31. http://dx.doi.org/10.1176/ajp.146.4.529

215. American Society of Addiction Medicine. The ASAM national practice guideline for the use of medications in the treatment of addiction involving opioid use. Chevy Chase, MD: American Society of Addiction Medicine; 2015. http://www.asam.org/docs/default-source/practicesupport/guidelines-and-consensus-docs/national-practice-guideline. pdf?sfvrsn=22

216. Fiellin DA, Schottenfeld RS, Cutter CJ, Moore BA, Barry DT, O'Connor PG. Primary care-based buprenorphine taper vs maintenance therapy for prescription opioid dependence: a randomized clinical trial. JAMA Intern Med 2014;174:1947-54. http://dx.doi.org/10.1001/ jamainternmed.2014.5302

217. Weiss RD, Potter JS, Fiellin DA, et al. Adjunctive counseling during brief and extended buprenorphine-naloxone treatment for prescription opioid dependence: a 2-phase randomized controlled trial. Arch Gen Psychiatry 2011;68:1238-46. http://dx.doi.org/10.1001/ archgenpsychiatry.2011.121

218. Jones CM, Campopiano M, Baldwin G, McCance-Katz E. National and state treatment need and capacity for opioid agonist medicationassisted treatment. Am J Public Health 2015;105:e55-63. http://dx.doi. org/10.2105/AJPH.2015.302664
219. Mark TL, Lubran R, McCance-Katz EF, Chalk M, Richardson J. Medicaid coverage of medications to treat alcohol and opioid dependence. J Subst Abuse Treat 2015;55:1-5. http://dx.doi. org/10.1016/j.jsat.2015.04.009

220. Minozzi S, Amato L, Vecchi S, Davoli M, Kirchmayer U, Verster A. Oral naltrexone maintenance treatment for opioid dependence. Cochrane Database Syst Rev 2006:1:CD001333.

221. Krupitsky E, Nunes EV, Ling W, Illeperuma A, Gastfriend DR, Silverman BL. Injectable extended-release naltrexone for opioid dependence: a double-blind, placebo-controlled, multicentre randomised trial. Lancet 2011;377:1506-13. http://dx.doi. org/10.1016/S0140-6736(11)60358-9

222. Substance Abuse and Mental Health Services Administration. Physician waiver qualifications. Rockville, MD: US Department of Health and Human Services, Substance Abuse and Mental Health Services Administration; 2015. http://www.samhsa.gov/medication-assistedtreatment/buprenorphine-waiver-management

223. Reuben DB, Alvanzo AAH, Ashikaga T, et al. National Institutes of Health Pathways to Prevention Workshop: the role of opioids in the treatment of chronic pain. Ann Intern Med 2015;162:295-300. http:// dx.doi.org/10.7326/M14-2775 
TABLE 1. Grading of Recommendations Assessment, Development and Evaluation (GRADE) clinical evidence review ratings of the evidence for the key clinical questions regarding effectiveness and risks of long-term opioid therapy for chronic pain

\begin{tabular}{|c|c|c|c|c|c|c|c|}
\hline Outcome & Studies & Limitations & Inconsistency & Imprecision & $\begin{array}{l}\text { Type of } \\
\text { evidence }\end{array}$ & Other factors & Estimates of effect/findings \\
\hline \multicolumn{8}{|c|}{ Effectiveness and comparative effectiveness (KQ1) } \\
\hline \multicolumn{8}{|c|}{ Effectiveness of long-term opioid therapy versus placebo or no opioid therapy for long-term ( $\geq 1$ year) outcomes } \\
\hline $\begin{array}{l}\text { Pain, function, and } \\
\text { quality of life }\end{array}$ & None & $-^{\dagger}$ & - & - & Insufficient & - & No evidence \\
\hline \multicolumn{8}{|c|}{ Harms and adverse events (KQ2) } \\
\hline \multicolumn{8}{|c|}{ Risks of opioids versus placebo or no opioids on opioid abuse, addiction, and related outcomes; overdose; and other harms } \\
\hline Abuse or addiction & $\begin{array}{r}1 \text { cohort study } \\
(\mathrm{n}=568,640)\end{array}$ & $\begin{array}{l}\text { Serious } \\
\text { limitations }\end{array}$ & $\begin{array}{l}\text { Unknown (1 } \\
\text { study) }\end{array}$ & No imprecision & 3 & None identified & $\begin{array}{l}\text { One retrospective cohort study found } \\
\text { long-term use of prescribed opioids } \\
\text { associated with an increased risk of abuse } \\
\text { or dependence diagnosis versus no opioid } \\
\text { use (adjusted OR ranged from } 14.9 \text { to } \\
122.5 \text {, depending on dose). }\end{array}$ \\
\hline Abuse or addiction & $\begin{array}{l}10 \text { uncontrolled studies } \\
(\mathrm{n}=3,780)\end{array}$ & $\begin{array}{l}\text { Very serious } \\
\text { limitations }\end{array}$ & $\begin{array}{l}\text { Very serious } \\
\text { inconsistency }\end{array}$ & No imprecision & 4 & None identified & $\begin{array}{l}\text { In primary care settings, prevalence of } \\
\text { opioid abuse ranged from } 0.6 \% \text { to } 8 \% \text { and } \\
\text { prevalence of dependence from } 3 \% \text { to } \\
26 \% \text {. In pain clinic settings, prevalence of } \\
\text { misuse ranged from } 8 \% \text { to } 16 \% \text { and } \\
\text { addiction from } 2 \% \text { to } 14 \% \text {. Prevalence of } \\
\text { aberrant drug-related behaviors ranged } \\
\text { from } 6 \% \text { to } 37 \% \text {. }\end{array}$ \\
\hline Overdose & $\begin{array}{l}1 \text { cohort study } \\
(\mathrm{n}=9,940)\end{array}$ & $\begin{array}{l}\text { Serious } \\
\text { limitations }\end{array}$ & $\begin{array}{l}\text { Unknown (1 } \\
\text { study) }\end{array}$ & $\begin{array}{l}\text { Serious } \\
\text { imprecision }\end{array}$ & 3 & None identified & $\begin{array}{l}\text { Current opioid use associated with } \\
\text { increased risk of any overdose events } \\
\text { (adjusted } \mathrm{HR} 5.2,95 \% \mathrm{Cl}=2.1-12 \text { ) and } \\
\text { serious overdose events (adjusted HR 8.4, } \\
95 \% \mathrm{Cl}=2.5-28 \text { ) versus current nonuse. }\end{array}$ \\
\hline Fractures & $\begin{array}{l}1 \text { cohort study } \\
\text { ( } \mathrm{n}=2,341) \text { and } \\
1 \text { case-control study } \\
\text { ( } \mathrm{n}=21,739 \text { case } \\
\text { patients) }\end{array}$ & $\begin{array}{l}\text { Serious } \\
\text { limitations }\end{array}$ & No inconsistency & No imprecision & 3 & None identified & $\begin{array}{l}\text { Opioid use associated with increased risk of } \\
\text { fracture in } 1 \text { cohort study (adjusted HR } \\
1.28,95 \% \mathrm{Cl}=0.99-1.64 \text { ) and } 1 \\
\text { case-control study (adjusted OR 1.27, } \\
95 \% \mathrm{Cl}=1.21-1.33 \text { ). }\end{array}$ \\
\hline Myocardial infarction & $\begin{array}{l}1 \text { cohort study } \\
\text { ( } \mathrm{n}=426,124) \text { and } \\
1 \text { case-control study } \\
\text { ( } \mathrm{n}=11,693 \text { case } \\
\text { patients) }\end{array}$ & No limitations & No inconsistency & No imprecision & 3 & None identified & $\begin{array}{l}\text { Current opioid use associated with } \\
\text { increased risk of myocardial infarction } \\
\text { versus nonuse (adjusted OR } 1.28 \text {, } \\
95 \% \mathrm{Cl}=1.19-1.37 \text { and incidence rate } \\
\text { ratio } 2.66,95 \% \mathrm{Cl}=2.30-3.08 \text { ). }\end{array}$ \\
\hline Endocrinologic harms & $\begin{array}{l}1 \text { cross-sectional study } \\
(\mathrm{n}=11,327)\end{array}$ & $\begin{array}{l}\text { Serious } \\
\text { limitations }\end{array}$ & $\begin{array}{l}\text { Unknown (1 } \\
\text { study) }\end{array}$ & No imprecision & 3 & None identified & $\begin{array}{l}\text { Long-term opioid use associated with } \\
\text { increased risk for use of medications for } \\
\text { erectile dysfunction or testosterone } \\
\text { replacement versus nonuse (adjusted OR } \\
1.5,95 \% \mathrm{Cl}=1.1-1.9 \text { ). }\end{array}$ \\
\hline \multicolumn{8}{|c|}{ How do harms vary depending on the opioid dose used? } \\
\hline Abuse or addiction & $\begin{array}{r}1 \text { cohort study } \\
(\mathrm{n}=568,640)\end{array}$ & $\begin{array}{l}\text { Serious } \\
\text { limitations }\end{array}$ & $\begin{array}{l}\text { Unknown (1 } \\
\text { study) }\end{array}$ & No imprecision & 3 & None identified & $\begin{array}{l}\text { One retrospective cohort study found } \\
\text { higher doses of long-term opioid therapy } \\
\text { associated with increased risk of opioid } \\
\text { abuse or dependence than lower doses. } \\
\text { Compared to no opioid prescription, the } \\
\text { adjusted odds ratios were } 15 \\
(95 \% \mathrm{Cl}=10-21) \text { for } 1 \text { to } 36 \mathrm{MME} / \mathrm{day}, 29 \\
(95 \% \mathrm{Cl}=20-41) \text { for } 36 \text { to } 120 \mathrm{MME} / \text { day, } \\
\text { and } 122(95 \% \mathrm{Cl}=73-205) \text { for } \\
\geq 120 \mathrm{MME} / \text { day. }\end{array}$ \\
\hline Overdose & $\begin{array}{l}1 \text { cohort study } \\
\text { ( } \mathrm{n}=9,940) \text { and } \\
1 \text { case-control study } \\
\text { ( } \mathrm{n}=593 \text { case patients } \\
\text { in primary analysis) }\end{array}$ & $\begin{array}{l}\text { Serious } \\
\text { limitations }\end{array}$ & No inconsistency & No imprecision & 3 & $\begin{array}{l}\text { Magnitude of } \\
\text { effect, dose } \\
\text { response } \\
\text { relationship }\end{array}$ & $\begin{array}{l}\text { Versus } 1 \text { to }<20 \mathrm{MME} / \text { day, one cohort study } \\
\text { found an adjusted } \mathrm{HR} \text { for an overdose } \\
\text { event of } 1.44(95 \% \mathrm{Cl}=0.57-3.62) \text { for } 20 \\
\text { to }<50 \mathrm{MME} / \text { day that increased to } 8.87 \\
(95 \% \mathrm{Cl}=3.99-19.72) \text { at } \geq 100 \mathrm{MME} / \text { day; } \\
\text { one case-control study found an adjusted } \\
\text { OR for an opioid-related death of } 1.32 \\
(95 \% \mathrm{Cl}=0.94-1.84) \text { for } 20 \text { to } 49 \mathrm{MME} / \text { day } \\
\text { that increased to } 2.88(95 \% \mathrm{Cl}=1.79-4.63) \\
\text { at } \geq 200 \mathrm{MME} \text { /day. }\end{array}$ \\
\hline Fractures & $\begin{array}{l}1 \text { cohort study } \\
(\mathrm{n}=2,341)\end{array}$ & $\begin{array}{l}\text { Serious } \\
\text { limitations }\end{array}$ & $\begin{array}{l}\text { Unknown (1 } \\
\text { study) }\end{array}$ & $\begin{array}{l}\text { Serious } \\
\text { imprecision }\end{array}$ & 3 & None identified & $\begin{array}{l}\text { Risk of fracture increased from an adjusted } \\
\mathrm{HR} \text { of } 1.20(95 \% \mathrm{Cl}=0.92-1.56) \text { at } 1 \text { to }<20 \\
\mathrm{MME} / \text { day to } 2.00(95 \% \mathrm{Cl}=1.24-3.24) \text { at } \\
\geq 50 \mathrm{MME} / \text { day; the trend was of borderline } \\
\text { statistical significance. }\end{array}$ \\
\hline
\end{tabular}

See table footnotes on page 47. 
TABLE 1. (Continued) Grading of Recommendations Assessment, Development and Evaluation (GRADE) clinical evidence review ratings of the evidence for the key clinical questions regarding effectiveness and risks of long-term opioid therapy for chronic pain

\begin{tabular}{|c|c|c|c|c|c|c|c|}
\hline Outcome & Studies & Limitations & Inconsistency & Imprecision & $\begin{array}{l}\text { Type of } \\
\text { evidence }\end{array}$ & Other factors & Estimates of effect/findings \\
\hline Myocardial infarction & $\begin{array}{r}1 \text { cohort study } \\
(n=426,124)\end{array}$ & $\begin{array}{l}\text { Serious } \\
\text { limitations }\end{array}$ & $\begin{array}{l}\text { Unknown } \\
\text { (1 study) }\end{array}$ & No imprecision & 3 & None identified & $\begin{array}{l}\text { Relative to a cumulative dose of } 0 \text { to } 1,350 \\
\text { MME during a } 90 \text {-day period, the } \\
\text { incidence rate ratio for myocardial } \\
\text { infarction for } 1350 \text { to }<2700 \mathrm{MME} \text { was } 1.21 \\
(95 \% \mathrm{Cl}=1.02-1.45) \text {, for } 2,700 \text { to }<8,100 \\
\mathrm{MME} \text { was } 1.42(95 \% \mathrm{Cl}=1.21-1.67) \text {, for } \\
8,100 \text { to }<18,000 \mathrm{MME} \text { was } 1.89 \\
(95 \% \mathrm{Cl}=1.54-2.33) \text {, and for } \geq 18,000 \mathrm{MME} \\
\text { was } 1.73(95 \% \mathrm{Cl}=1.32-2.26) \text {. }\end{array}$ \\
\hline $\begin{array}{l}\text { Motor vehicle crash } \\
\text { injuries }\end{array}$ & $\begin{array}{l}1 \text { case-control study } \\
\text { ( } n=5,300 \text { case } \\
\text { patients) }\end{array}$ & No limitations & $\begin{array}{l}\text { Unknown } \\
\text { (1 study) }\end{array}$ & No imprecision & 3 & None identified & $\begin{array}{l}\text { No association between opioid dose and } \\
\text { risk of motor vehicle crash injuries even } \\
\text { though opioid doses }>20 \mathrm{MME} / \text { day were } \\
\text { associated with increased odds of road } \\
\text { trauma among drivers. }\end{array}$ \\
\hline Endocrinologic harms & $\begin{array}{l}1 \text { cross-sectional study } \\
(\mathrm{n}=11,327) \text { New for } \\
\text { update: } 1 \text { additional } \\
\text { cross-sectional study } \\
(\mathrm{n}=1,585)\end{array}$ & $\begin{array}{l}\text { Serious } \\
\text { limitations }\end{array}$ & Consistent & No imprecision & 3 & None identified & $\begin{array}{l}\text { Relative to } 0 \text { to }<20 \mathrm{MME} / \text { day, the adjusted } \\
\text { OR for } \geq 120 \mathrm{MME} / \text { day for use of } \\
\text { medications for erectile dysfunction or } \\
\text { testosterone replacement was } 1.6 \\
(95 \% \mathrm{Cl}=1.0-2.4) \text {. } \\
\text { One new cross-sectional study found } \\
\text { higher-dose long-term opioid therapy } \\
\text { associated with increased risk of androgen } \\
\text { deficiency among men receiving } \\
\text { immediate-release opioids (adjusted OR } \\
\text { per } 10 \mathrm{MME} / \text { day } 1.16,95 \% \mathrm{Cl}=1.09-1.23) \text {, } \\
\text { but the dose response was very weak } \\
\text { among men receiving ER/LA opioids. }\end{array}$ \\
\hline \multicolumn{8}{|l|}{ Dosing strategies (KQ3) } \\
\hline \multicolumn{8}{|c|}{ Comparative effectiveness of different methods for initiating opioid therapy and titrating doses } \\
\hline Pain & $\begin{array}{l}3 \text { randomized trials } \\
(n=93)\end{array}$ & $\begin{array}{l}\text { Serious } \\
\text { limitations }\end{array}$ & $\begin{array}{l}\text { Serious } \\
\text { inconsistency }\end{array}$ & $\begin{array}{l}\text { Very serious } \\
\text { imprecision }\end{array}$ & 4 & None identified & $\begin{array}{l}\text { Trials on effects of titration with immediate- } \\
\text { release versus ER/LA opioids reported } \\
\text { inconsistent results and had additional } \\
\text { differences between treatment arms in } \\
\text { dosing protocols (titrated versus fixed } \\
\text { dosing) and doses of opioids used. }\end{array}$ \\
\hline Overdose & $\begin{array}{l}\text { New for update: } \\
1 \text { cohort study } \\
(n=840,606)\end{array}$ & $\begin{array}{l}\text { Serious } \\
\text { limitations }\end{array}$ & $\begin{array}{l}\text { Unknown } \\
\text { (1 study) }\end{array}$ & No imprecision & 4 & None identified & $\begin{array}{l}\text { One new cross-sectional study found } \\
\text { initiation of therapy with an ER/LA opioid } \\
\text { associated with increased risk of overdose } \\
\text { versus initiation with an immediate- } \\
\text { release opioid (adjusted HR } 2.33 \text {, } \\
95 \% \mathrm{Cl}=1.26-4.32 \text { ). }\end{array}$ \\
\hline \multicolumn{8}{|c|}{ Comparative effectiveness of different ER/LA opioids } \\
\hline Pain and function & $\begin{array}{l}3 \text { randomized trials } \\
(\mathrm{n}=1,850)\end{array}$ & $\begin{array}{l}\text { Serious } \\
\text { limitations }\end{array}$ & No inconsistency & No imprecision & 3 & None identified & No differences \\
\hline All-cause mortality & $\begin{array}{l}1 \text { cohort study } \\
(n=108,492) \\
\text { New for update: } \\
1 \text { cohort study } \\
(n=38,756)\end{array}$ & $\begin{array}{l}\text { Serious } \\
\text { limitations }\end{array}$ & $\begin{array}{l}\text { Serious } \\
\text { inconsistency }\end{array}$ & No imprecision & 4 & None identified & $\begin{array}{l}\text { One cohort study found methadone to be } \\
\text { associated with lower all-cause mortality } \\
\text { risk than sustained-release morphine in a } \\
\text { propensity-adjusted analysis (adjusted HR } \\
0.56,95 \% \mathrm{Cl}=0.51-0.62 \text { ) and one cohort } \\
\text { study among Tennessee Medicaid patients } \\
\text { found methadone to be associated with } \\
\text { higher risk of all-cause mortality than } \\
\text { sustained-release morphine (adjusted HR } \\
1.46,95 \% \mathrm{Cl}=1.17-1.73 \text { ). }\end{array}$ \\
\hline $\begin{array}{l}\text { Abuse and related } \\
\text { outcomes }\end{array}$ & $\begin{array}{l}1 \text { cohort study } \\
(n=5,684)\end{array}$ & $\begin{array}{l}\text { Serious } \\
\text { limitations }\end{array}$ & $\begin{array}{r}\text { Unknown } \\
\text { (1 study) }\end{array}$ & $\begin{array}{l}\text { Serious } \\
\text { imprecision }\end{array}$ & 4 & None identified & $\begin{array}{l}\text { One cohort study found some differences } \\
\text { between ER/LA opioids in rates of adverse } \\
\text { outcomes related to abuse, but outcomes } \\
\text { were nonspecific for opioid-related } \\
\text { adverse events, precluding reliable } \\
\text { conclusions. }\end{array}$ \\
\hline \multicolumn{8}{|c|}{ ER/LA versus immediate-release opioids } \\
\hline Endocrinologic harms & $\begin{array}{l}\text { New for update: } \\
1 \text { cross-sectional } \\
\text { study }(n=1,585)\end{array}$ & $\begin{array}{l}\text { Serious } \\
\text { limitations }\end{array}$ & $\begin{array}{r}\text { Unknown } \\
\text { (1 study) }\end{array}$ & No imprecision & 4 & None identified & $\begin{array}{l}\text { One cross-sectional study found ER/LA } \\
\text { opioids associated with increased risk of } \\
\text { androgen deficiency versus immediate- } \\
\text { release opioids (adjusted OR 3.39, } \\
95 \% \mathrm{Cl}=2.39-4.77 \text { ). }\end{array}$ \\
\hline
\end{tabular}

See table footnotes on page 47. 
TABLE 1. (Continued) Grading of Recommendations Assessment, Development and Evaluation (GRADE) clinical evidence review ratings of the evidence for the key clinical questions regarding effectiveness and risks of long-term opioid therapy for chronic pain

\begin{tabular}{|c|c|c|c|c|c|c|c|}
\hline Outcome & Studies & Limitations & Inconsistency & Imprecision & $\begin{array}{l}\text { Type of } \\
\text { evidence }\end{array}$ & Other factors & Estimates of effect/findings \\
\hline \multicolumn{8}{|c|}{ Dose escalation versus dose maintenance or use of dose thresholds } \\
\hline $\begin{array}{l}\text { Pain, function, or } \\
\text { withdrawal due to } \\
\text { opioid misuse }\end{array}$ & $\begin{array}{l}1 \text { randomized trial } \\
(n=140)\end{array}$ & $\begin{array}{l}\text { Serious } \\
\text { limitations }\end{array}$ & $\begin{array}{l}\text { Unknown } \\
\text { (1 study) }\end{array}$ & $\begin{array}{l}\text { Very serious } \\
\text { imprecision }\end{array}$ & 3 & None identified & $\begin{array}{l}\text { No difference between more liberal dose } \\
\text { escalation versus maintenance of current } \\
\text { doses in pain, function, or risk of } \\
\text { withdrawal due to opioid misuse, but } \\
\text { there was limited separation in opioid } \\
\text { doses between groups ( } 52 \text { versus } 40 \\
\text { MME/day at the end of the trial). }\end{array}$ \\
\hline
\end{tabular}

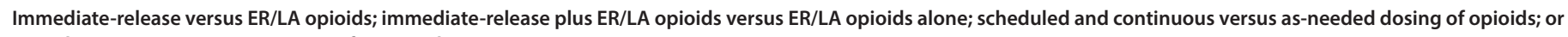
opioid rotation versus maintenance of current therapy

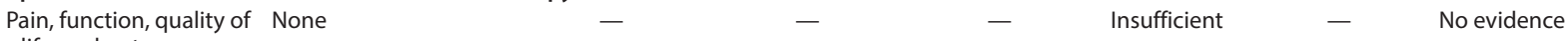

life, and outcomes

related to abuse

Effects of decreasing or tapering opioid doses versus continuation of opioid therapy

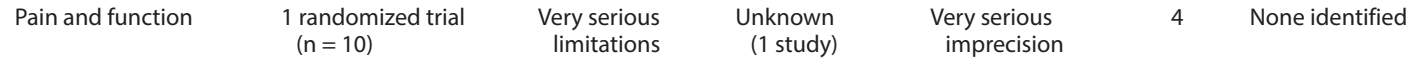

Comparative effectiveness of different tapering protocols and strategies

Opioid abstinence $\quad 2$ nonrandomized trials Very serious

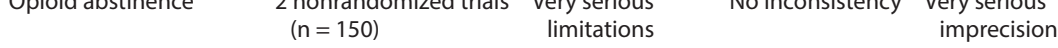

$4 \quad$ None identified
Abrupt cessation of morphine was associated with increased pain and decreased function compared with continuation of morphine.

Risk assessment and risk mitigation strategies (KQ4)

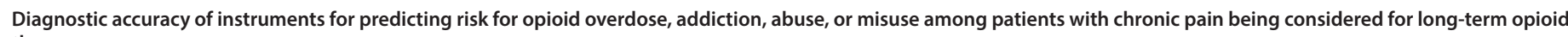
therapy

Opioid risk tool

$\begin{array}{lr}3 \text { studies of diagnostic } & \text { Serious } \\ \text { accuracy }(n=496) & \text { limitations } \\ \text { New for update: } & \\ 2 \text { studies of diagnostic } & \\ \text { accuracy }(n=320) & \\ 2 \text { studies of diagnostic } & \text { Very serious } \\ \text { accuracy }(n=203) & \text { limitations }\end{array}$

$\begin{array}{ll}\begin{array}{l}\text { Very serious } \\ \text { inconsistency }\end{array} & \text { Serious } \\ \text { imprecision }\end{array}$

No inconsistency Serious

Screener and Opioid 2 studies of diagnostic Very serious with Pain, Version 1 $\begin{array}{ccc}\begin{array}{c}\text { Screener and Opioid } \\ \begin{array}{l}\text { Assessment for Patients } \\ \text { with Pain-Revised }\end{array}\end{array} & \begin{array}{l}\text { New for update: } \\ \text { accuracy }(\mathrm{n}=320)\end{array} & \begin{array}{r}\text { Very serious } \\ \text { actimitations }\end{array} \\ \text { Brief Risk Interview } & \begin{array}{l}\text { New for update: } \\ 2 \text { studies of diagnostic } \\ \text { accuracy }(\mathrm{n}=320)\end{array} & \begin{array}{r}\text { Very serious } \\ \text { limitations }\end{array}\end{array}$
No inconsistency Serious imprecision

No inconsistency Serious imprecision
$4 \quad$ None identified

None

None identified

imprecision
No clear differences between different methods for opioid discontinuation or tapering in likelihood of opioid abstinence after 3-6 months

See table footnotes on page 47. 
TABLE 1. (Continued) Grading of Recommendations Assessment, Development and Evaluation (GRADE) clinical evidence review ratings of the evidence for the key clinical questions regarding effectiveness and risks of long-term opioid therapy for chronic pain

\begin{tabular}{|c|c|c|c|c|c|c|c|}
\hline Outcome & Studies & Limitations & Inconsistency & Imprecision & $\begin{array}{c}\text { Type of } \\
\text { evidence }\end{array}$ & Other factors & Estimates of effect/findings \\
\hline \multicolumn{8}{|c|}{ Effectiveness of risk prediction instruments on outcomes related to overdose, addiction, abuse, or misuse in patients with chronic pain } \\
\hline $\begin{array}{l}\text { Outcomes related to } \\
\text { abuse }\end{array}$ & None & - & - & - & Insufficient & - & No evidence \\
\hline \multicolumn{8}{|c|}{ 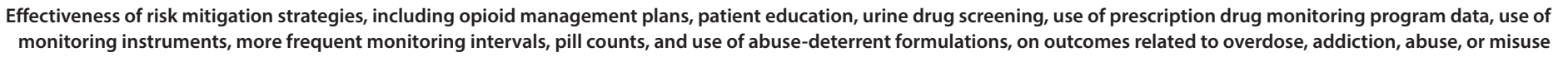 } \\
\hline $\begin{array}{l}\text { Outcomes related to } \\
\text { abuse }\end{array}$ & None & - & - & - & Insufficient & - & No evidence \\
\hline \multicolumn{8}{|c|}{ Effectiveness of risk prediction instruments on outcomes related to overdose, addiction, abuse, or misuse in patients with chronic pain } \\
\hline $\begin{array}{l}\text { Outcomes related to } \\
\text { abuse }\end{array}$ & None & - & - & - & Insufficient & - & No evidence \\
\hline \multicolumn{8}{|c|}{ 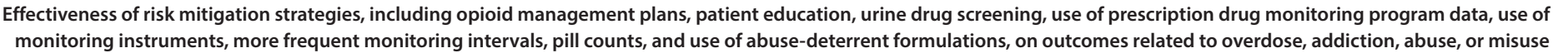 } \\
\hline $\begin{array}{l}\text { Outcomes related to } \\
\text { abuse }\end{array}$ & None & - & - & - & Insufficient & - & No evidence \\
\hline \multicolumn{8}{|c|}{ Comparative effectiveness of treatment strategies for managing patients with addiction to prescription opioids } \\
\hline $\begin{array}{l}\text { Outcomes related to } \\
\text { abuse }\end{array}$ & None & - & - & - & Insufficient & - & No evidence \\
\hline \multicolumn{8}{|c|}{ Effects of opioid therapy for acute pain on long-term use (KQ5) } \\
\hline Long-term opioid use & $\begin{array}{l}\text { New for update: } \\
2 \text { cohort studies } \\
\text { (n=399,852) }\end{array}$ & $\begin{array}{l}\text { Serious } \\
\text { limitations }\end{array}$ & No inconsistency & No imprecision & 3 & None identified & $\begin{array}{l}\text { One study found use of opioids within } \\
7 \text { days of low-risk surgery associated with } \\
\text { increased likelihood of opioid use at } 1 \text { year } \\
\text { (adjusted OR } 1.44,95 \% \mathrm{Cl}=1.39-1.50 \text { ), } \\
\text { and one study found use of opioids within } \\
15 \text { days of onset of low back pain among } \\
\text { workers with a compensation claim } \\
\text { associated with increased risk of late } \\
\text { opioid use (adjusted OR } 2.08 \text {, } \\
95 \% \mathrm{Cl}=1.55-2.78 \text { for } 1 \text { to } 140 \mathrm{MME} / \text { day } \\
\text { and OR } 6.14,95 \% \mathrm{Cl}=4.92-7.66 \text { for } \\
\geq 450 \mathrm{MME} / \text { day). }\end{array}$ \\
\hline
\end{tabular}

Abbreviations: $\mathrm{Cl}$ = confidence interval; $\mathrm{ER} / \mathrm{LA}=$ extended release/long-acting; $\mathrm{HR}$ = hazard ratio; $\mathrm{MME}$ = morphine milligram equivalents; $\mathrm{OR}=$ odds ratio.

* Ratings were made per GRADE quality assessment criteria; "no limitations" indicates that limitations assessed through the GRADE method were not identified.

${ }^{\dagger}$ Not applicable as no evidence was available for rating. 
TABLE 2. Morphine milligram equivalent (MME) doses for commonly prescribed opioids

\begin{tabular}{lc}
\hline Opioid & Conversion factor* $^{*}$ \\
\hline Codeine & 0.15 \\
Fentanyl transdermal (in mcg/hr) & 2.4 \\
Hydrocodone & 1 \\
Hydromorphone & 4 \\
Methadone & \\
$1-20$ mg/day & 4 \\
$21-40$ mg/day & 8 \\
$41-60$ mg/day & 10 \\
$\geq 61-80$ mg/day & 12 \\
Morphine & 1 \\
Oxycodone & 1.5 \\
Oxymorphone & 3 \\
Tapentadol & 0.4 \\
\hline
\end{tabular}

Source: Adapted from Von Korff M, Saunders K, Ray GT, et al. Clin J Pain 2008;24:521-7 and Washington State Interagency Guideline on Prescribing Opioids for Pain (http://www.agencymeddirectors.wa.gov/ Files/2015AMDGOpioidGuideline.pdf).

* Multiply the dose for each opioid by the conversion factor to determine the dose in MMEs. For example, tablets containing hydrocodone $5 \mathrm{mg}$ and acetaminophen $300 \mathrm{mg}$ taken four times a day would contain a total of $20 \mathrm{mg}$ of hydrocodone daily, equivalent to $20 \mathrm{MME}$ daily; extended-release tablets containing oxycodone $10 \mathrm{mg}$ and taken twice a day would contain a total of $20 \mathrm{mg}$ of oxycodone daily, equivalent to $30 \mathrm{MME}$ daily. The following cautions should be noted: 1) All doses are in $\mathrm{mg} /$ day except for fentanyl, which is $\mathrm{mcg} /$ hr. 2) Equianalgesic dose conversions are only estimates and cannot account for individual variability in genetics and pharmacokinetics. 3) Do not use the calculated dose in MMEs to determine the doses to use when converting opioid to another; when converting opioids the new opioid is typically dosed at substantially lower than the calculated MME dose to avoid accidental overdose due to incomplete cross-tolerance and individual variability in opioid pharmacokinetics.4) Use particular caution with methadone dose conversions because the conversion factor increases at higher doses. 5) Use particular caution with fentanyl since it is dosed in $\mathrm{mcg} / \mathrm{hr}$ instead of $\mathrm{mg} / \mathrm{day}$, and its absorption is affected by heat and other factors.

† Tapentadol is a mu receptor agonist and norepinephrine reuptake inhibitor. MMEs are based on degree of mu-receptor agonist activity, but it is unknown if this drug is associated with overdose in the same dose-dependent manner as observed with medications that are solely mu receptor agonists. 
Recommendations and Reports

\section{Steering Committee and Core Expert Group Members}

Steering Committee: Deborah Dowell, MD, Tamara M. Haegerich, PhD; Division of Unintentional Injury Prevention, National Center for Injury Prevention and Control, CDC; Roger Chou, MD; on detail to CDC under contract.

Core Expert Group Members: Pam Archer, MPH, Oklahoma State Department of Health; Jane Ballantyne, MD; University of Washington (retired); Amy Bohnert, PhD; University of Michigan; Bonnie Burman, ScD; Ohio Department on Aging; Roger Chou, MD; on detail to CDC under contract; Phillip Coffin, MD, San Francisco Department of Public Health; Gary Franklin, MD, MPH; Washington State Department of Labor and Industries/University of Washington; Erin Krebs, MDH; Minneapolis VA Health Care System/University of Minnesota; Mitchel Mutter, MD, Tennessee Department of Health; Lewis Nelson, MD; New York University School of Medicine; Trupti Patel, MD, Arizona Department of Health Services; Christina A. Porucznik, PhD, University of Utah; Robert "Chuck" Rich, MD, FAAFP, American Academy of Family Physicians; Joanna Starrels, MD, Albert Einstein College of Medicine of Yeshiva University; Michael Steinman, MD, Society of General Internal Medicine; Thomas Tape, MD, American College of Physicians; Judith Turner, PhD, University of Washington.

\section{Stakeholder Review Group}

John Markman, MD, American Academy of Neurology; Bob Twillman, PhD, American Academy of Pain Management; Edward C. Covington, MD, American Academy of Pain Medicine; Roger F. Suchyta, MD, FAAP, American Academy of Pediatrics; Kavitha V. Neerukonda, JD, American Academy of Physical Medicine and Rehabilitation; Mark Fleury, PhD, American Cancer Society Cancer Action Network; Penney Cowan, American Chronic Pain Association; David Juurlink, BPharm, MD, PhD, American College of Medical Toxicology; Gerald "Jerry" F. Joseph, Jr, MD, American College of Obstetrics and Gynecology; Bruce Ferrell, MD, AGSF, M. Carrington Reid, MD, PhD, American Geriatrics Society; Ashley Thompson, American Hospital Association; Barry D. Dickinson, PhD, American Medical Association; Gregory Terman MD, PhD, American Pain Society; Beth Haynes, MPPA, American Society of Addiction Medicine; Asokumar Buvanendran, MD, American Society of Anesthesiologists; Robert M. Plovnick; MD, American Society of Hematology; Sanford M. Silverman, MD, American Society of Interventional Pain Physicians; Andrew Kolodny, MD, Physicians for Responsible Opioid Prescribing.

Chair: Christina Porucznik, PhD, MSPH

\section{Opioid Guideline Workgroup}

Workgroup Members: Anne Burns, RPh; Penney Cowan; Chinazo Cunningham, MD, MS; Katherine Galluzzi, DO; Traci Green, PhD, MSC; Mitchell Katz, MD; Erin Krebs, MD, MPH; Gregory Terman, MD, PhD; Mark Wallace, MD. Workgroup Consultants: Roger Chou, MD; Edward Covington, MD; Diana Eppolito; Michael Greene, MD; Steven Stanos, DO.

\section{Peer Reviewers}

Jeanmarie Perrone, MD, University of Pennsylvania; Matthew Bair, MD, Indiana University School of Medicine; David Tauben, MD, University of Washington.

\section{NCIPC Board of Scientific Counselors}

Chair: Stephen Hargarten, MD, MPH; Members: John Allegrante, PhD; Joan Marie Duwve, MD, Samuel Forjuoh, MD, MPH, DrPH, FGCP; Gerard Gioia, PhD; Deborah Gorman-Smith, PhD; Traci Green, PhD; Sherry Lynne Hamby, PhD; Robert Johnson, MD; Angela Mickalide, PhD, MCHES; Sherry Molock, PhD; Christina Porucznik, PhD, MSPH; Jay Silverman, PhD; Maria Testa, PhD; Shelly Timmons, MD, PhD, FACS, FAANS; Ex Officio Members: Melissa Brodowski, PhD; Dawn Castillo, MPH; Wilson Compton, MD, MPE; Elizabeth Edgerton, MD, MPH; Thomas Feucht, PhD; Meredith Fox, PhD; Holly Hedegaard, MD, MSPH; John Howard, MD; Lyndon Joseph, PhD; Jinhee Lee, PharmD; Iris Mabry-Hernandez, MD, MPH; Valeri Maholmes, PhD; Angela Moore Parmley, PhD; Thomas Schroeder, MS. 
The Morbidity and Mortality Weekly Report (MMWR) Series is prepared by the Centers for Disease Control and Prevention (CDC) and is available free of charge in electronic format. To receive an electronic copy each week, visit MMWR's free subscription page at http://www.cdc.gov/mmwr/mmwrsubscribe.html. Paper copy subscriptions are available through the Superintendent of Documents, U.S. Government Printing Office, Washington, DC 20402; telephone 202-512-1800.

Readers who have difficulty accessing this PDF file may access the HTML file at http://www.cdc.gov/mmwr/volumes/65/rr/rr6501e1.htm?s_cid=rr6501e1_w. htm. Address all inquiries about the $M M W R$ Series, including material to be considered for publication, to Executive Editor, $M M W R$ Series, Mailstop E-90, CDC, 1600 Clifton Rd., N.E., Atlanta, GA 30329-4027 or to mmwrq@cdc.gov.

All material in the MMWR Series is in the public domain and may be used and reprinted without permission; citation as to source, however, is appreciated. Use of trade names and commercial sources is for identification only and does not imply endorsement by the U.S. Department of Health and Human Services.

References to non-CDC sites on the Internet are provided as a service to $M M W R$ readers and do not constitute or imply endorsement of these organizations or their programs by CDC or the U.S. Department of Health and Human Services. CDC is not responsible for the content of these sites. URL addresses listed in $M M W R$ were current as of the date of publication.

ISSN: $1057-5987$ (Print) 